\title{
An Evaluation of Extended Duration Multi-touch Interaction
}

\author{
Shiroq Al-Megren \\ Supervisor: Prof. Patrick Olivier \\ MSc. Computing Science \\ School of Computing Science \\ University of Newcastle upon Tyne \\ August 26 ${ }^{\text {th }}, 2011$
}




\begin{abstract}
The goal of this project is to evaluate the extended use of multi-touch interaction techniques, more specifically the ergonomic convenience of existing bimanual and unimanual interaction techniques and personal preference over an extended period of time for both horizontal and vertical tabletops. Objective localized muscle fatigue, muscle activity, and subjective perceived exertion measures were administrated. In the experimental design, electromyograms were recorded during tabletop interaction technique and voluntary isometric contractions were recorded pre- and post-tabletop activity for the biceps brachii, middle deltoid, and extensor digitorum for both sides of the body. Changes in the median power frequency (MPF) and root mean square (RMS) were explored to examine muscular fatigue and activity respectively. MPF was found sensitive to fatigue for some muscles on both the horizontal and vertical condition where a decline in MPF was noted, albeit statistically insignificant. Perceived exertion ratings have shown an increase by the end of the task where the difference between the means was found to be statistically significant for the vertical condition but not the horizontal one. The electromyograms recordings, along with video recordings, have shown the sustainability of the interaction techniques adopted at the beginning of the task to the end of that task, which included both unimanual and bimanual techniques.
\end{abstract}




\section{Declaration}

I declare that this dissertation represents my own work except where otherwise stated. 


\section{Acknowledgments}

I would like to thank Prof. Patrick Olivier, my project supervisor, for his support, guidance and encouragement throughout this thesis. My gratitude also goes to Dr. Ahmed Kharrufa for articulating the project's idea and for his support during the project. Special thank goes to everyone in Space 2 in Culture Lab for their willingness to lend a hand whenever it was needed.

I am sincerely and heartily grateful to my husband for his love and unconditional support throughout this thesis. A special thanks goes to my parents and siblings for their prayers and their complete faith in my capabilities. 


\section{Table of Contents}

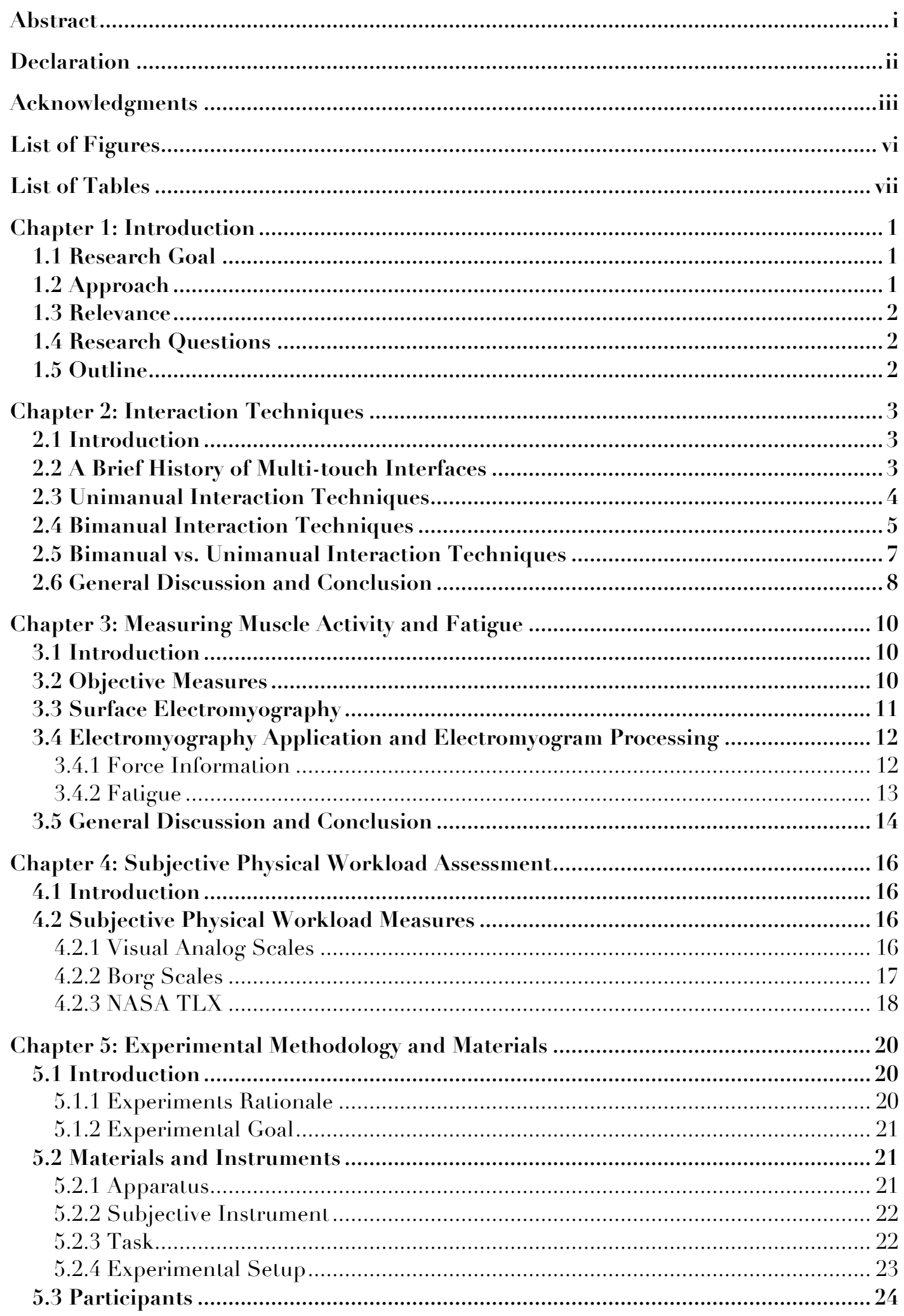




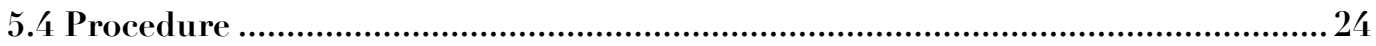

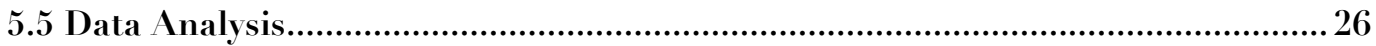

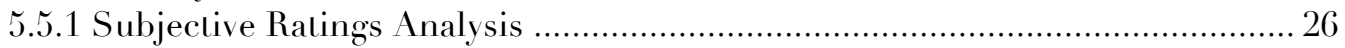

5.5.2 Isometric Contraction Electromyogram Analysis ............................................ 26

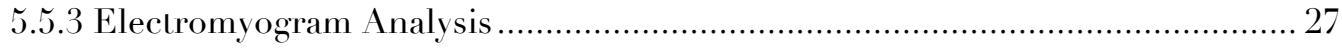

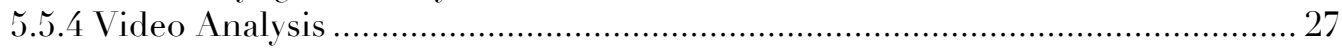

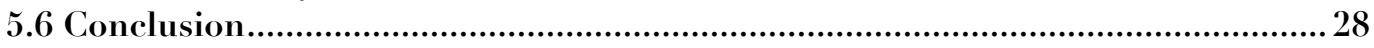

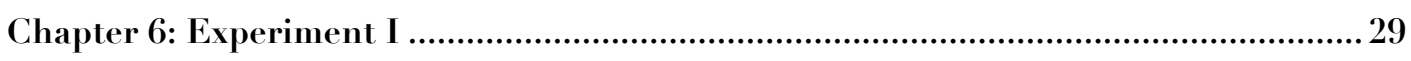

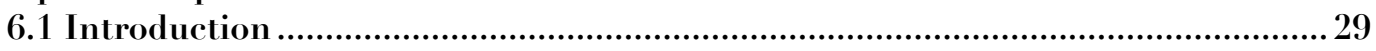

6.2 Purpose of the Experiment..................................................................................29

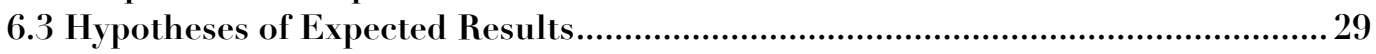

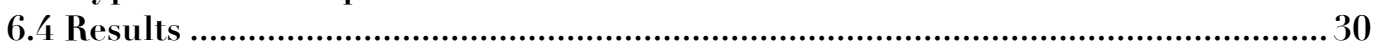

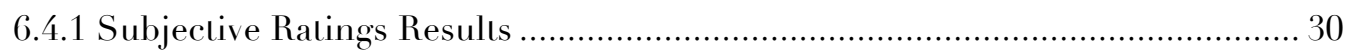

6.4.2 Isometric Contraction Electromyogram Results ............................................... 30

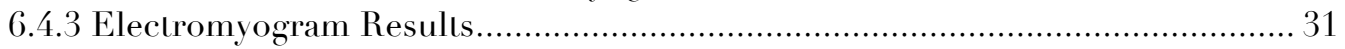

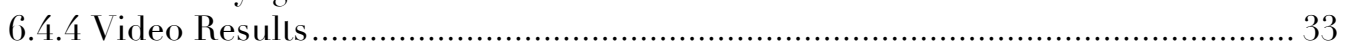

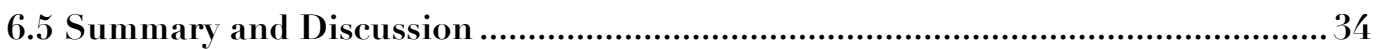

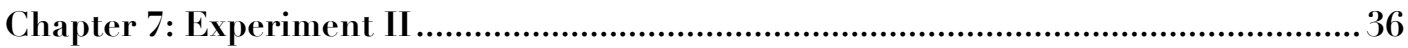

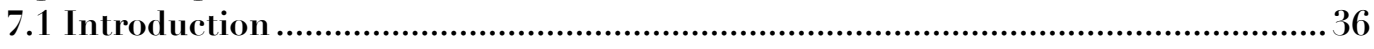

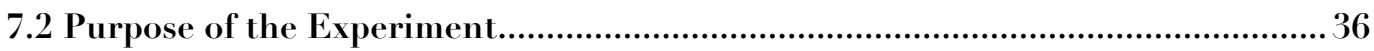

7.3 Hypotheses of Expected Results............................................................................

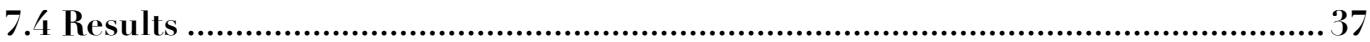

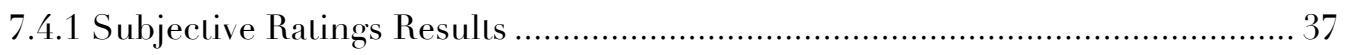

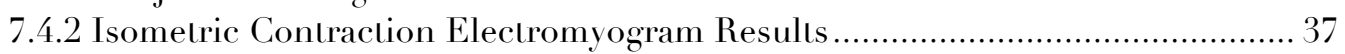

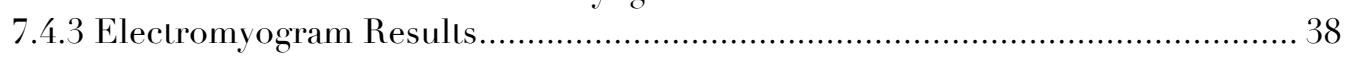

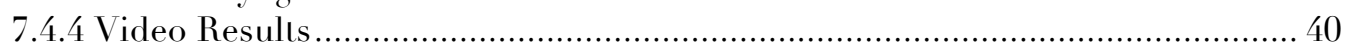

7.5 Summary and Discussion ................................................................................... 41

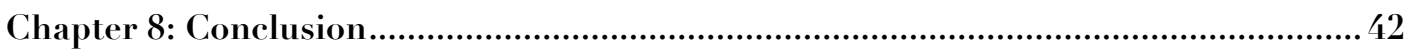

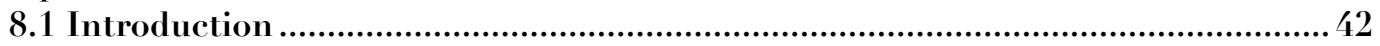

8.2 Answering the Research Questions ……………................................................ 42

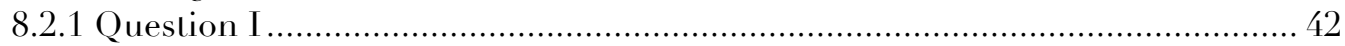

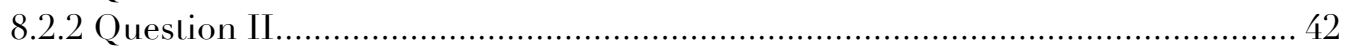

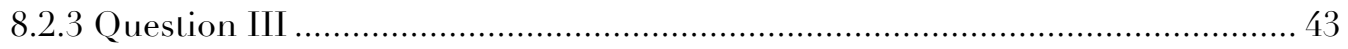

8.3 Critical Remarks on the Experiments ................................................................... 43

8.4 Summary of Conclusion ........................................................................................ 44

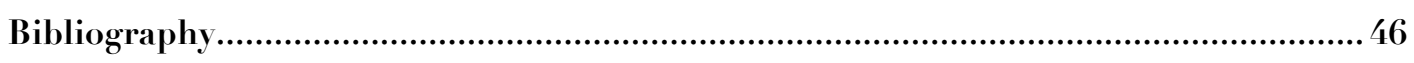

Appendix A: Experiment Materials ..............................................................................53

A.1 Isometric Contraction Electromyogram Analysis ..................................................53

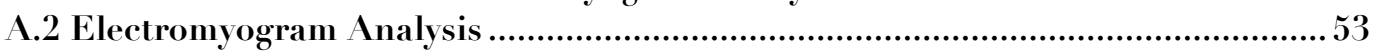

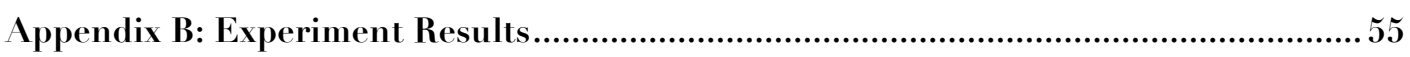

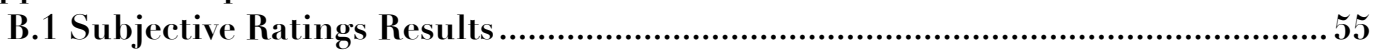

B.2 Isometric Contraction Electromyogram Results ........................................56

B.2.1 Isometric Contraction Results for the Horizontal Condition........................... 56

B.2.2 Isometric Contraction Results for the Vertical Condition .............................. 58

B.3 Electromyogram ANOVA Results .........................................................................60

B.3.1 Electromyogram ANOVA Results for the Horizontal Condition ...................... 60

B.3.2 Electromyogram ANOVA Results for the Vertical Condition.......................... 61

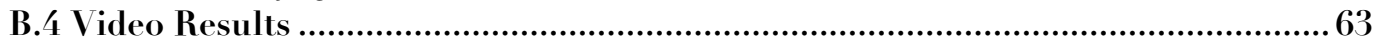




\section{List of Figures}

Figure 5-1: the right panel shows the ScatterPuzzle developed by Microsoft and left panel shows the modified application used in the study

Figure 5-3: the experimental setup for the horizontal (right) and the vertical (left) conditions

Figure 5-4: the placements of the electrodes for the middle deltoid (right), biceps brachii

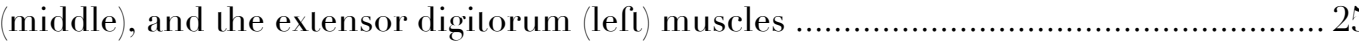

Figure 5-5: adopted postures to record isometric contractions for the middle deltoid (left), biceps brachii (middle), and the extensor digitorum (right) muscles ...................... 25

Figure 6-1: normalized MPF for the isometric contractions recorded for the six muscles after the task; shown as a percentage of the isometric contraction recorded before the task.

Figure 6-2: normalized RMS measured for the middle deltoids during the experimental task as a function of block ....

Figure 6-3: normalized RMS measured for the biceps brachii during the experimental task as a function of block ....

Figure 6-4: normalized RMS measured for the extensor digitorum during the experimental task as a function of block

Figure 6-5: the resting postures adopted during the experimental task. The top panel shows the left (non-dominant) arm resting on the side of the tabletop. The bottom panel shows both arms resting on top of the tabletop while interactions continues to ensue 33

Figure 7-1: normalized MPF for the isometric contractions recorded for the six muscles after the task shown as a percentage of the isometric contraction recorded before the task.

Figure 7-2: normalized RMS measured for the middle deltoids during the experimental task as a function of block

Figure 7-3: normalized RMS measured for the biceps brachii during the experimental task as a function of block ....

Figure 7-4: normalized RMS measured for the extensor digitorum during the experimental task as a function of block

Figure 7-5: the posture adopted by 3 of the 6 subjects participating in the experiment, where the crossed leg is used as a resting place for the left (non-dominant) arm ........ 40

Figure B-1: the subjective ratings collected before and after the experimental task for the horizontal condition

Figure B-2: the subjective ratings collected before and after the experimental task for the vertical condition 


\section{List of Tables}

Table 5-1: the time blocks and their designated time during task, all of a length of 1 minute

Table B-1: paired t-test results for the left middle deltoid isometric contraction pre- and

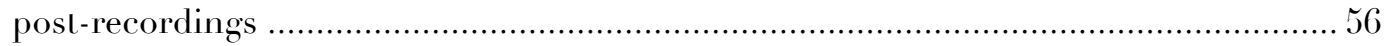

Table B-2: paired t-test results for the right middle deltoid isometric contraction pre- and

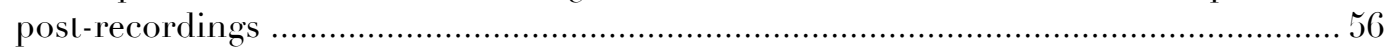

Table B-3: paired t-test results for the left biceps brachii isometric contraction pre- and

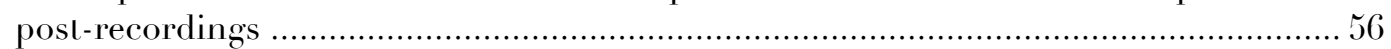

Table B-4: paired t-test results for the right biceps brachii isometric contraction pre- and

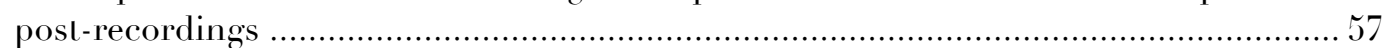

Table B-5: paired t-test results for the left extensor digitorum isometric contraction pre- and

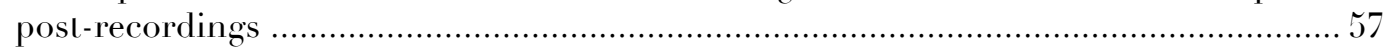

Table B-6: paired t-test results for the right extensor digitorum isometric contraction pre-

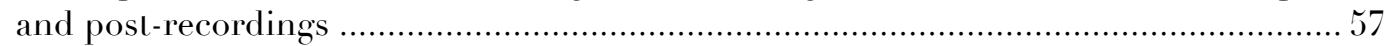

Table B-7: paired t-test results for the left middle deltoid isometric contraction pre- and

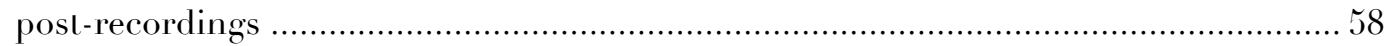

Table B-8: paired t-test results for the right middle deltoid isometric contraction pre- and

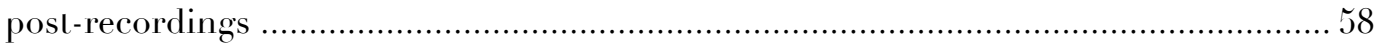

Table B-9: paired t-test results for the left biceps brachii isometric contraction pre- and

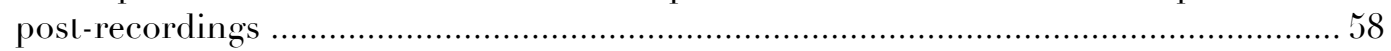

Table B-10: paired t-test results for the right biceps brachii isometric contraction pre- and post-recordings

Table B-11: paired t-test results for the left extensor digitorum isometric contraction pre-

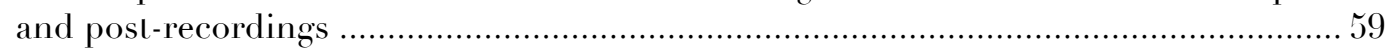

Table B-12: paired t-test results for the right extensor digitorum isometric contraction pre-

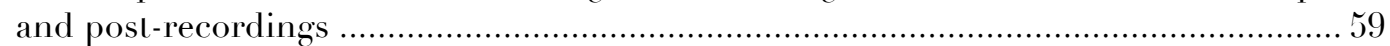

Table B-13: ANOVA results for the left middle deltoid electromyograms recorded during the task

Table B-14: ANOVA results for the right middle deltoid electromyograms recorded during the task

Table B-15: ANOVA results for the left biceps brachii electromyograms recorded during the task.

Table B-16: ANOVA results for the right biceps brachii electromyograms recorded during the task

Table B-17: ANOVA results for the left extensor digitorum electromyograms recorded during the task

Table B-18: ANOVA results for the right extensor digitorum electromyograms recorded during the task

Table B-19: ANOVA results for the left middle deltoid electromyograms recorded during the task

Table B-20: ANOVA results for the right middle deltoid electromyograms recorded during the task

Table B-21: ANOVA results for the left biceps brachii electromyograms recorded during the task.

Table B-22: ANOVA results for right biceps brachii electromyograms recorded during the task

Table B-23: ANOVA results for the left extensor digitorum electromyograms recorded

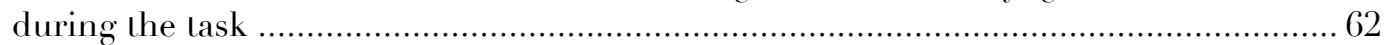

Table B-24: ANOVA results for the right extensor digitorum electromyograms recorded during the task 


\section{Chapter 1}

\section{Introduction}

In 1945, long before traditional workstations, Bush envisioned memex, an interactive electromechanical device for record production, storage and consultation. Memex was foreseen as "[...] a desk, and while it can presumably be operated from a distance, it is primarily the piece of furniture at which he works. On the top are slanting translucent screens, on which material can be projected for convenient reading. There is a keyboard, and sets of buttons and levers. Otherwise it looks like an ordinary desk." [1] In 1993, Wellner pioneered Bush's vision with DigitalDesk; a desk from which a computer display is projected and video cameras aimed at in order to detect tactical interactions [2]. The innovative design is able to detect input from fingers and pens, thus enabling touch interactions, and can also sense and recognize documents placed on it. The term tabletop has commonly been used in research to signify the location of the display: on a table or a desk.

Tabletops and touch interaction migrate their users from traditional desktop workstations with restricted interactive manipulations to more intuitive interactive techniques. This transition is exemplified commercially with multi-touch tabletops such as Microsoft's Surface and Mitsubishi's DiamondTouch [3]. Such displays incorporate a number of interaction techniques that allow users to interact either unimanually using one or multiple-fingers, or bimanually.

The novelty of multi-touch interaction techniques is appealingly portrayed in science fiction literature and movies with little scientific merit of its ergonomic feasibility. Although a considerable amount of research has gone into tabletops the numerous facets of tabletops, e.g. long-term use [4-6], most are conducted with minimal attention has been paid towards the ergonomics of its interaction techniques over an extended period of time.

This thesis presents investigative research of the ergonomic feasibility of horizontal and vertical tabletops and in particular, users' interaction technique preference.

\subsection{Research Goal}

The goal of this project is to evaluate the extended use of multi-touch unimanual and bimanual interaction techniques as well as the ergonomic convenience of existing interaction techniques when employed over an extended period of time for both horizontal and vertical large surfaces. The thesis aims to conduct the evaluation subjectively and objectively to allow for valid interpretation of fatigability and the subjective employment of the interaction techniques.

\subsection{Approach}

Few quantitative assessments, be they subjective or objective, of tabletop and multi-touch interaction technique fatigability and preference over time have been conducted in the human-computer interaction discipline. In pursuit of the thesis goal and ergonomic gap in tabletop literature, commonly employed unimanual and bimanual interaction techniques have been surveyed. An exploration of ergonomic subjective and objective measures for the 
assessment of fatigue and muscle activity was then ensued with justifiable selections. After the assessment criterion had been established and interaction techniques selected for this thesis, a testing implementation supporting rotation, translation and scaling of surface elements was developed. This was followed by the design of a study that aimed to evaluate extended use of the interaction techniques selected over a long period of time. Finally, insights were gathered, analyzed and discussed in both investigated conditions.

\subsection{Relevance}

Current workstation designs are not aimed at the ergonomics of use but the convenience of production. Work-related upper extremity musculoskeletal disorders (MSD) have surged due to the dissemination of traditional workstations imposing physical and financial disabilities to those afflicted Since then, ergonomic knowledge has expanded and careful consideration of the design of interaction techniques for traditional workstations has grown. As tabletops and multi-touch interaction techniques show promise in their enrichment of interaction in various fields, it is crucial to assess their effects on the musculoskeletal system to avoid work-related MSD. The research goal presented is partly aimed at objectively assessing predominant interaction techniques that have yet to disseminate to the larger population in everyday use. The second part of the research aims to evaluate the user preference of tabletop interaction techniques over an extended period of time. This preference provides insight into the long-term sustainability of the assessed interaction techniques. Ergo, the research goal is relevant to the field of human-computer interaction and ergonomics.

\subsection{Research Questions}

The experiments have been conducted with three questions to be answered:

I. What interaction techniques are adopted for tabletop interactions when unimanual and bimanual techniques are made available?

II. Are the adopted techniques, be they bimanual or unimanual, sustainable throughout the hour-long task?

III. Are tabletop interaction techniques fatiguing over an hour-long task?

In this thesis, we hope to improve the quality of interaction techniques by assessing their fatigability and sustainability over time through providing insight into their utilization for an hour-long activity in the form of means of subjective and objective measures.

\subsection{Outline}

In the following chapter, literature on bimanual and unimanual interaction techniques is reviewed. Chapter 3 gives an overview of objective measures of muscle activity and fatigue, a selection falls on electromyography as the measure of choice, therefore the physiological phenomena and its utilization over various fields is reviewed. Chapter 4 considers subjective measures for the assessment of perceived physical workload shadowed by the selection of an instrument for application in this thesis. Chapter 5 highlights the methodologies and materials chosen for the evaluation of tabletop interaction techniques in horizontal and vertical conditions. In chapter 6 and 7, two user studies investigating tabletop interaction techniques over an extended period of time are presented. The thesis generally concludes in chapter 8 . 


\section{Chapter 2}

\section{Interaction Techniques}

\subsection{Introduction}

This chapter offers a review of prior research, relevant to unimanual and bimanual interaction techniques. Firstly, a brief description of the historical background of multitouch interfaces is provided. Section 2.3 reviews unimanual interaction techniques research. An investigation of bimanual interaction techniques follows in section 2.4, which defines bimanual interaction based on the recruitment degree of both hands: symmetrical and asymmetrical. Section 2.5 reviews literature comparing unimanual and bimanual interaction techniques. The chapter concludes in section 2.6, with a critical discussion and summary of the preceding sections.

\subsection{A Brief History of Multi-touch Interfaces}

Human computer interaction research works towards facilitating communication between humans and computers through constrictive communication bandwidth. For instance, traditional desktop workstations restrict user interaction by limiting the scope of actions used to operate the workstation and reducing the user to an "eye and a finger" [7]. A great deal of time is spent on workstations, facilitating interactions with them can allow for a more fluid integration into everyday use, no longer hampering but supporting. An interaction technique, as defined by Jacob $[8]$, is " $[\ldots]$ a way of using a physical input/output device to perform a generic task in a human-computer dialogue." The dominant techniques for traditional workstations fall under several interaction styles: command line, menu selection, and direct manipulation; of which, direct manipulation is the most prominent since the introduction of graphical displays $[9]$. With direct manipulation, the translation of intention to manipulation is visible, for example, moving a document from one folder to another in a graphical user interface. Direct manipulation is continuously evolving and is extended to newer concepts such as multi-touch interfaces, tangible user interfaces, and virtual reality. Due to their germaneness to the thesis goal, multi-touch interfaces will be further elucidated.

As the name implies, multi-touch interfaces recognize two or more touches simultaneously, feasibly accommodating two or more people interacting with the touch system. This form of interaction is becoming of the user's intuitive behavior and the naturalness of contact and manipulation. For instance, a user may view an image on the touch display and then rotate it and translate to an opposite collaborator; such behavior is a digital facsimile of real-world interactions. Multi-touch technology dates back to 1982 with the Flexible Machine Interface developed by Nimish Mehta for his masters's thesis at the University of Toronto. In 1984 at Bell Labs, Boie developed the first multi-touch screen that enabled the manipulation of graphical objects permitted by a transparent array of capacitors overlaid over a cathode ray tube $[10]$. Similarly, Kasday patented a technological invention for touch sensing that uses optical methods in the same year [11]. A year later at the University of Toronto, a group that included William Buxton, presented a multi-touch tablet with different degrees of contact by dividing the screen into a grid of distinct points sensed by a scanning algorithm [12]. Later, in the early 1990's, Wellner [2] pioneered 
DigitalDesk, which used a projector to project a display onto a desk where cameras detect the users hand manipulations of the display elements. It was also noted by Saffer [7], that DigitalDesk is the first multi-touch interactive device that used some of today's familiar interactive gestures, such as pinch to shrink and spread to expand.

The 1990's and early 2000s saw an increase in multi-touch research carried out on handsets, tablets, and tables. In 1992, Buxton introduced flip board: a combined keyboard and touch tablet that aimed to extend the capabilities of existing direct manipulation systems [13]. In the same year, Wacom unveiled a series of digital tablets named UD that made it possible to sense multiple devices (stylus and puck) that facilitated bimanual interaction. Successive generations of the series, further extend the UD's multi-point capability for location, tilt, and rotation sensing for the stylus and puck [14]. In the mid 1990's Liu, as part of the Input Research Group from the University of Toronto, developed a prototype system of a drafting table with a projected display, which captures the position, pose, and orientation of the user's hands, as the dominant hand interacts with a stylus, thus enabling bimanual interaction. Buxton identifies Liu's development, Active Desk, as "[...] an early version of what has become known as tabletop or surface computing" [15]. In 2001, Mitsubishi unveiled DiamondTouch; their contribution distinguished between the different collaborators interactions [3]. Microsoft later introduced PlayAnywhere in 2005, an interactive tabletop, which uses a practical implementation of computer vision [16].

Multi-touch technology continued to evolve with little everyday use until recently. In 2007, Apple Inc. introduced the iPhone and iPod Touch, with a capacitive touch screen for multi-touch sensing. It was then that the notion of multi-touch displays was popularized commercially. The introduction of the iPhone to the market and its widespread adoption lead other chief handset manufacturers, such as Nokia and Sony Ericsson, to launch their own touchscreen handsets [7]. Microsoft later released MS Surface, a tabletop technology with a multi-touch surface that senses interactions made by bare hands and/or objects. Microsoft surface is further described in Chapter 5.

\subsection{Unimanual Interaction Techniques}

Unimanual interaction techniques involve one or more fingers on one hand for manipulations, such as navigating and selecting an icon using a mouse. Research in unimanual interaction mainly fall under two categories of comparison: between direct and indirect input, and unimanual and bimanual interaction techniques. The latter case is discussed in Section 2.5. For the former, Sears et al. [17] investigated touchscreen and mouse input for a high resolution selection task. And the study has shown that with selection targets at a width of 16 pixels or more, the touchscreen selection performed better than the mouse input, yet subjective measures showed a preference for mouse input over direct-touch. Sears et al. [17] concluded that a problem which has continually arisen with touchscreens is arm fatigue, which was not noted nor assessed in the study. In a similar study, Forlines et al. [18] conducted two experiments for selection and dragging tasks on a tabletop, only one of which is relevant to this section. The results of that study showed that direct-touch of targets with a size of $1.92 \mathrm{~cm}$ or higher outperformed mouse input, although with an increased error rate.

Meyer et al. [19] compared five input devices in a task that required dragging, pointing, zooming, text entry and menu selection on a desktop display, completed on the basis of written instructions given to subjects. The five input devices compared were: mouse, mouse pen, trackball, touchscreen, and stylus. Meyer et al. [19] reported mean completion time for all input devices where the mouse ranked the highest with performance and the touchscreen the lowest, with the longest completion time. The subjectively perceived performances of the mouse and touchscreen proved identical for mean completion time. Of the five devices, reports of fatigue were associated with the touchscreen and trackball. 
Multi-finger interaction can also be observed for unimanual interaction techniques. Moscovich et al. [20] explored multi-finger unimanual and bimanual interaction techniques on a touchscreen and touchpad with two experiments. The first experiment aimed to map finger movements against visual response to confirm compatibility using a technique that allows the translation, rotation, and scaling of an object simultaneously. Of the two techniques, multi-finger unimanual proved effective in translation and rotation of objects. The second experiment evaluated an object alignment task for the same interaction techniques from the first experiment for structure assessment. Unlike the first experiment, the multi-finger bimanual interaction technique outperformed its unimanual counterpart.

The next section discusses the second area of research: interaction design and empirical studies of symmetrical and asymmetrical bimanual interaction techniques.

\subsection{Bimanual Interaction Techniques}

Bimanual interaction techniques employ both hands to provide intention for manipulation. There has been substantial research in the area of bimanual interaction in terms of empirical studies and interaction design. One of the earliest empirical studies was that of Buxton et al. [21]. The bimanual investigation was carried out due to the general belief that bimanualism improves interaction performance, as it is evident in the employment of both hands in everyday tasks, such as changing gears while steering the car. Their hypothesis was tested with two controlled studies: the first involved positioning/scaling tasks, the second involved a comparative word selection assessment in which unimanual and bimanual techniques are compared. For the first study, bimanualism was intuitively used by all but one subject with no indication of additional load to the cognitive system. It was also noted that the tasks were carried out in parallel by most subjects positively correlating with their performances. The results of the second study showed that the bimanual technique increased performance rates as the task was divided between the two hands reducing the gap between expert and novice users; albeit less parallelism was observed compared to the first study which is attributed by the examiners to the difficulty of the task this study still resulted in better completion times. The observed results of the two experiments provided evidence of the increased efficiency of bimanual interactions in manipulation tasks with no additional cognitive load for the evaluation task.

The review of subsequent literature categorizes bimanual interaction research into two classes: bimanual asymmetric and symmetric interaction techniques.

Guiard's Kinematic Chain $(\mathrm{KC})$ model logically describes the division of labour that directs bimanual behavior. It assumes that the two hands are a pair of motors assembled to produce a uniform mechanical effect. Guiard states that three ways to assemble the two motors exist: orthogonally, in parallel and serially. An orthogonal assembly involves the two motors governing two separate motions that are orthogonal to one another. With a parallel assembly, the two motors are in synergy controlling the same motor proportion. Two motors that are assembled in a series work on the same motion, where one motor consumes the product of the other motor. He further explains that a serial assembly is what human hands mostly exhibit and thus models bimanual interaction asymmetrically. The assembly suggests that within the non-preferred hand frame of reference, the preferred hand can perform frequent actions as the non-preferred hand performs infrequent actions [22]. The model has since guided the investigation and evaluation of bimanual interaction techniques.

Toolglass, a bimanual interaction technique designed by Bier et al. [23], is a collection of semi-transparent widgets and sheets that are used bimanually and in coordination with Magic Lenses to perform tasks on a selected object highlighted by the sheet's selection. With the new style, the non-preferred hand can position the sheet over an object, thus setting a frame of reference, while the dominant hand manipulates. An empirical study 
carried out by Kabbash et al. [24] evaluated Toolglass, as one of the asymmetrical techniques, against other asymmetric, unimanual, and symmetric bimanual techniques. The task developed for evaluation required the drawing of colored line segments between dots by matching the chosen drawing color with the next dot to appear i.e. selection of color followed by a dragging operation from one dot to another to draw the line. The result of the study has shown that the Toolglass technique took less time to complete as it involved fewer motor operations and reduced cognitive load. The authors have also noted that bimanual techniques are not always better than unimanual ones, which was similarly observed by Zhai et al. [25]. However, appropriately designed bimanual techniques that utilize existing skills can prove to be superior to other techniques.

Subsequently, Balakrishnan et al. [26] designed a digital tape drawing system used for sketching on large vertical displays. In the automotive industry, tape drawing is used to conceptually sketch car designs using black photographic tape on large vertical displays. It was observed that the skill of tape drawing uses both hands asymmetrically, where the nondominant hand unrolls the tape and the dominant hand slides along the tape and then fastens it to the surface of the drawing display. Tape drawing artists from different automotive companies evaluated the digitized system over different periods of time, and the results have shown an intuitive adoption of the system by the artists as it emulated an existing skill. Grossman et al. [27] [28] later augmented the previously designed system [26] for tape drawing. One such extension was the ability to sketch a design unimanually, as bimanualism proved unsuitable for situations that were biomechanically limited. Their design and further extension addresses the importance of designing with the interactive task in mind, where the suitability of the interaction is closely linked to the technique.

Prior to the development of Balakrishnan et al. [26] digital tape drawing system, an asymmetrical bimanual system for free-form surface drawing was developed by Shaw et al. [29]. Similarly the system was designed for curvature drawing but unlike the digital tape drawing system [26], THRED consists of a traditional workstation with a screen, keyboard, and mouse with two additional 3D sensors. The system asymmetrically divided the effort of sketching to both hands according to Guiard's Kinematic Chain [22]; the non-dominant hand sets the framework where the dominant hand performs finer manipulations, mainly point selection and reshaping.

Research in bimanual input has mainly focused on asymmetric interaction techniques guided by Guiard's Kinematic Chain [22]. Nevertheless, there are activities, as pointed out by Guiard, that are not asymmetrical, but in fact symmetrical in nature. In symmetrical activities both hands work together with equal levels of importance at the same time, such as folding a sheet or skipping rope [30]. A few studies have examined the potential benefits of symmetric bimanual interaction techniques, for example, Leganchuk et al. [31] which observed the benefits of symmetrical techniques over asymmetric bimanual and unimanual techniques for an area sweeping task. The increased benefit was attributed to mental load reduction and the increased degrees of freedom. For rectangle editing and navigation, Casalta et al. [32], symmetrical techniques performed better with a higher degree of parallelism than the asymmetrical techniques. Owen et al. [33] empirically compared bimanual and unimanual interaction techniques for a curve matching task. The study has shown that the task took less time to complete with the symmetrical bimanual condition, which was attributed, by the examiners, to the difficulty of the task.

Several symmetric bimanual interaction techniques have been designed, and are reviewed in human-computer interaction literature. Hinckley et al. [34] described and evaluated bimanual input devices for map navigation, one of which is symmetrical. For 3D navigation, Zhai et al. [35] proposed a symmetrical bimanual bulldozer-like technique. An experimental evaluation was conducted on the proposed technique and has shown the objective and subjective outperformance of the designed technique over a traditional unimanual technique, which was, in part, attributed to the bimanualism of the technique. A symmetrical bimanual curve manipulation interaction technique, similar to the task used in 
[33] and its asymmetrical equivalent [26-28], symSpline is designed by Latulipe et al. [36]. An experimental evaluation of symSpline against bimanual and unimanual techniques was carried out and the results have shown that the symmetrical technique outperformed the other techniques, subjectively and objectively.

The suitability of symmetric or asymmetric bimanual interaction techniques is highly dependent on the manipulation task. For curve manipulation, both symmetrical [33][36] and asymmetrical [26-28] techniques were proposed, with positive adaptation and positive evaluated outcomes. Symmetrical techniques were also developed for 2D and 3D navigation [34][35] and selection/dragging [23-25], and visualization [37] tasks have had their fair share of asymmetrical techniques, proving superior over other unimanual and bimanual techniques.

\subsection{Bimanual vs. Unimanual Interaction Techniques}

Input efficiency of unimanual and bimanual techniques has been comparatively investigated in a number of studies carried out in the aforementioned literature. Section 2.4 detailed the work of Buxton et al. [21] which has shown bimanual interaction techniques to be superior over unimanual techniques in performance efficiency with no additional cognitive load for the evaluation task. In Section 2.4, the work of Kabbash et al. [24] was abridged, which outlined the evaluation of one unimanual technique against three bimanual techniques, one of which was Toolglass [23]. In their experiment, the task was performed by each of the four techniques and contrasted using two indicators: completion time and motor operations. The results show that the Toolglass technique outperformed the other three techniques as it had the least number of motor units and required less time to complete. Kabbash et al. [24] also noted that Toolglass was the only bimanual technique that outperformed the unimanual technique, which resolved that the design of the bimanual technique is essential to its efficiency.

Hinckley et al. [38] investigated the possible advantages that bimanual interaction techniques can bring to simulated manipulation. The task designed for evaluation involved the alignment of objects unimanually and bimanually using passive props designed by Hinckley et al. [37] for neurosurgical visualization. For the bimanual technique, the two props can be handled simultaneously, whereas in the unimanual technique only one prop was handled at a time. After task completion, the subjects were asked to reproduce the dominant hand placement for which the accuracy of the angle and distance between the remembered and ideal reference frames were then quantitatively analyzed. The study results have shown that the unimanual condition imposed difficulties that bimanual techniques overcame. Those difficulties included future anticipation of the next move and the absence of a reference frame; most subjects in the unimanual condition had to rely on the surface as a reference to complete the task.

In another study, Leganchuck et al. [31] compared two bimanual and one unimanual techniques in two separate experiments. The first, involved the translation and the scaling of a geometric shape to enclose other predefined shapes using three techniques: a traditional unimanual graphical user interface technique, Toolglass [23], and a stretching symmetric bimanual technique. The results of the first experiment have shown the superiority of the bimanual techniques over the unimanual technique, with significantly higher mean completion times. The second experiment replicated the first following a practice period and compared the unimanual technique against the Toolglass bimanual technique. The results have shown that the gap between the times it took to complete the task bimanually and unimanually increased as the manipulation was practiced before hand. The quantitative results have also supported Leganchuck et al. [31] hypothesis that the outperformance gap between the unimanual and bimanual techniques will widen as the task becomes more cognitively difficult. 
The work of Latulipe et al. [39] compared the performance of unimanual, symmetric bimanual, and asymmetric bimanual techniques in an image registration task. The task involved the alignment of one or more images to form a whole; the registration technique used, entails the rotation, scaling, and translation of the image parts in order to complete the whole. The mean completion times for the three techniques were analyzed and have shown that the time it took to complete the task bimanually was shorter than the unimanual condition. Latulipe et al. [39] compared the bimanual techniques and reports that it took longer for the task to be completed asymmetrically in the bimanual condition than for it to be completed in the symmetrical condition. Subjective preferences were also collected after task completion in the study and of the 24 subjects, 22 preferred the symmetric bimanual technique.

The latest differential investigation of a multi-target selection task, conducted by Kin et al. [40], compared the performance efficiency of indirect unimanual, direct-touch unimanual, direct-touch with two fingers bimanual, unconstrained direct-touch bimanual technique using a mouse for the first condition, and a touch workstation for the directtouch conditions. The results of the study, similar to those in previously discussed studies albeit on a multi-target selection task, have shown superior completion time for the bimanual conditions. A strong preference for using one or two index fingers was also observed despite multi-touch viability. In contrast, for the translation and rotation of images, Moscovich et al. [20] observed better performances for two-fingered unimanual over two-fingered bimanual interaction techniques. Kin et al. [40] concluded with a set of design guidelines for the development of multi-touch workstations, where target selection is principal to the task: superiority of direct over indirect input and two-finger detection improve performance on touch stations. However, the detection of more than two fingers is deemed unnecessary; the feasibility of using traditional interfaces for direct-touch where the target is at least of the same size as a fingertip; the use of unimanual techniques for gestures and degree of freedom control and not for target selection.

\subsection{General Discussion and Conclusion}

A wealth of research is aimed at assessing the effectiveness of bimanual and unimanual interaction techniques. The studies detailed in Section 2.5 examined the advantages of bimanual interaction techniques, be they symmetrical or asymmetrical, over unimanual interaction techniques. The outperformance of bimanual techniques was attributed to task cognitive difficulty [31][33], parallelism [21][32], fewer motor operations [24], and the adoption of existing skills [23][26-28]. Instances where the performance of unimanual interaction techniques surpassed bimanual techniques have also been noted in the literature for simple selection tasks, for example in a selection and navigation task using two joysticks carried out by Xia, Irani, and Wang [41].

The prime focus of this thesis is to investigate unimanual and bimanual interaction techniques on tabletops over an extended period of time; it will also address the fatigability of the interaction techniques. Nevertheless, few studies have investigated user behavior towards both interaction techniques over an extended period of time or their fatigability. The majority of the research required participants to preform some constrained task repeatedly for a short period of time, not fully capturing normal interaction. In the case of fatigue, Meyer et al. [19] reported on subjective perceptions of fatigue for the unimanual interaction techniques adopted in their study for the touchscreen condition. Sears et al. [17] similarly noted the fatigability of touchscreen interaction technique. Buxton et al. [21] for their comparative investigation into bimanual interaction techniques for a compound selection/positioning task against a unimanual condition did not report ergonomic fatigue as the task was completed without fault in the bimanual condition. For long term horizontal or vertical use, Morris et al. [6] highlighted the discomfort reported by most subjects when using the horizontal surface for office use, whereas Wigdor et al. [4] stated 
that despite the fact that the display chosen was rather large, no reports of arm fatigue were noted by the subject.

In the next chapter, objective techniques for measuring fatigue and muscle activity are surveyed in this thesis. The technique selected will be used to address the fatigability and employment of unimanual and bimanual interaction techniques on vertical and horizontal tabletops. 


\section{Chapter 3}

\section{Measuring Muscle Activity and Fatigue}

\subsection{Introduction}

This chapter offers a review of prior research that is relevant to measuring fatigue objectively. Firstly, a brief description of objective measures employed for measuring fatigue is surveyed. It is followed by a selection in Section 3.3, which reviews surface electromyography historically. In Section 3.4, processing techniques and application of the surface electromyograph are assessed. Finally, section 3.5 justifies the selection of this objective measure and aforementioned processing techniques and summarizes the chapter.

\subsection{Objective Measures}

Since the detection of electrical current in muscle contractions and the invention of the galvanometer, the application of electromyography has been well established for measuring skeletal muscle activity over static and dynamic tasks. Thus in this section only prior research relevant to measuring fatigue is reviewed.

Physical fatigue is defined as "any exercise-induced reduction in the capacity to generate force or power output" [42]. Vøllestad [42] discusses different techniques to measure fatigue; the techniques are categorized as direct or indirect assessments based on the analysis of behavior or recorded perception respectively. The direct reported assessments are listed as follows: maximal voluntary force generation, power output, tetanic force, and low frequency fatigue. Maximal voluntary force generation is the most common and can be achieved by an isometric contraction (MVIC) where the subjects are instructed to achieve the highest possible force where force measurements are obtained. However, the technique faces several limitations, such as the difficulty to obtain maximal force from certain muscles. The assessment of maximal power output is quantified using ergometers or isokinetic devices. Fatigue in maximal power output is witnessed in the decline of the output in successive periods. Several studies have compared between MVIC and maximal power output where the overall fatigue for both proved to be compatible. Another measure of fatigue stimulates the nerves of the investigated muscle to produce measurable tetanic force, however, overstimulation can produce faulty results. The final direct measure put forth by Vøllestad [42] assesses twitch force where a fast decline is indicative of lowfrequency fatigue. Although, the direct measurement techniques listed above have been proven to be reliable indicators of fatigue, their use is unsuitable to the goal of this thesis as they require either clinical training and/or are applicably invasive.

Vøllestad [42] continues to report on indirect measures of fatigue which include: twitch interpolation, endurance time and electromyography. Twitch interpolation, similar to tetanic force and low-frequency fatigue, entails neuron stimulation to measure the level of muscle activation in a voluntary task. Another form of assessment measures endurance time, which is the ability to sustain force at target intensity for a certain period of time, however, the relationship between endurance time and fatigue is unreliable. The invasiveness of twitch interpolation and the unreliability of endurance time would not be suitable to measure fatigue in this thesis. The final indirect measure cited by Vollestad [42] 
is electromyography. Electromyography involves the detection of physiological changes to the skeletal muscles, thus it objectively measures muscle force during a task or a compound of tasks.

The next section describes surface electromyography and the necessary analysis for force quantification and fatigue indexing in the time and frequency domains.

\subsection{Surface Electromyography}

Since the 1940s, surface electromyography has been used for the detection of muscular activity during dynamic exercise as surface electrodes proved practical during movement, whereas needle and fine-wire electrodes can prove to be painfully invasive. For instance, in 1944, Inman, Sander, and Abbot measured shoulder muscle activation using surface electromyography as the shoulder was dynamically exercised. Subsequently, surface electromyography was adopted in clinical research that involved movement, most prominently, the work of Floyd and Silver on the erector spinae muscles. The inception of biofeedback in the 1960s can be attributed to the work conducted by Basmajian for training motor units using fine-wire electrodes. It was later in that decade that Green, Walter, Green et al. modified Basmajian motor unit training concept for its application for general relaxation exercises. The biofeedback researchers adoption of surface electromyography allowed its application for the treatment of neuromuscular disorders. For example, Budzynski et al. used biofeedback for the treatment of headaches caused by muscle contraction and in 1973 Johnson and Garton applied surface electromyography for functional restoration of hemiplegic subjects. Surface electromyography's contribution to measuring fatigue was noted by the works of Kadefors et al. and Masuda et al. in the year 1979 and 1999 respectively. The widespread adoption of surface electromyography in numerous fields necessitated the creation of foundations and organization for regulating its application, for instance the European - established Surface Electromyography for NonInvasive Study of Muscles (SENIAM) [43] to regulate application and electromyograph technology.

The necessity of cautiously applying surface electromyography in research has been popularly exemplified with De. Luca's following statement: "Electromyography is a seductive muse because it provides easy access to physiological processes that cause the muscle to generate force, produce movement and accomplish the countless functions which allow us to interact with the world around us. The current state of Surface Electromyography is enigmatic. It provides many important and useful applications, but it has many limitations, which must be understood, considered and eventually removed so that the discipline is more scientifically based and less reliant on the art of use. To its detriment, electromyography is too easy to use and consequently too easy to abuse." [44] Prior to approving surface electromyography as the objective measure for muscle activity and fatigue, its advantages and disadvantages should be thoroughly investigated.

The obvious advantage of surface electromyography lies in its practical application and noninvasiveness compared to needle or fine-wire electrodes. It is also safe for application with little or no side effects. For clinical application, it has significantly improved treatment approaches where the data obtained can be given to patients, and thus the patients' can adjust their responses appropriately to verbal commands [45]. In the field of ergonomics, surface electromyography has proven to be appropriate for the investigation of suspected muscle groups affected by the work place due to light yet repetitive movement [46].

The weaknesses of surface electromyography can be categorized into three classes: anatomy, instrumentation and method. An anatomical limitation lies in the inability to measure all muscle sites; the deeper the muscle the harder it is to detect and record activity with surface electrodes. Another anatomical and instrumentation concern is crosstalk, 
where the energy from one muscle interferes with the recording of another thus introducing the difficulty of isolating the signals for analysis. Crosstalk can be remedied by the electrode's degree of specificity and optimal placement. A second disadvantage due to the instrumentation adoption, despite technological advances, is its obstruction of natural behavior and thus altering the subject's posture or interaction. Over interpretation of electromyograph is one of the main concerns in the category of methods of analysis. For example, comparing data detected from one muscle with one recorded from another, where the interpreted difference could be due to muscle mass rather than the muscle's level of activity. Such comparison should only be attempted after normalizing the activity as a ratio of a referenced contraction [45]. An electromyogram is influenced by physiological and technical interferences; an understanding of both is necessary for sound interpretation of the signal. Electrode distance, size, and type can affect the signal, while volume of subcutaneous fat has also been associated with the signal-noise ratio. Physiological changes to the muscle during dynamic exercise can alter the propagation of the electrical impulse. For example, as the muscle lengthens, most likely due to changes to the sarcolemma membrane, the propagation of the electrical impulses alters the amplitude of the action potential and shifts the power spectrum towards lower frequencies [47].

\subsection{Electromyography Application and Electromyogram Processing}

The information obtained from an electromyogram can be processed to provide analytical interpretation in three categories: temporal information, force and fatigue. Temporal information assesses the activation and resting modes of a muscle and is considered to be the most basic information gathered from an electromyogram. The second category, force, attempts to quantify the muscle's electrical activity that is particular to that muscle's contraction, commonly as a percentage of a MVIC. The identification of localized muscular fatigue is the third category from which information can be obtained from an electromyogram $[46]$.

The subsequent subsections review the history of the application of electromygraphs and the processing that has ensued to obtain force and fatigue information from an electromyogram. Temporal information will not be discussed due to its irrelevance to the goal of this thesis.

\subsubsection{Force Information}

There exists a relationship between the electromyogram and muscle force, however, several factors are influential to that relationship such as acquisition procedure, kinematic movement and analytical processing. Lippold [48] carried out the first investigation into the relationship between the electromyogram and force by determining the manifestation of activity in certain muscles. Using a constructed dynamometer to produce voluntary isometric flexion of the calf and an electromyograph to record muscle activity during those contractions, Lippold [48] was then able to integrate three random action potential recordings to determine the relationship between the signal and force. The results have shown a linear relationship between the integrated electromyogram and the isometric tension with correlation coefficients varying from 0.93 and 0.99. Similarly, Close [49] suggested a linear relationship between the integrated electromyogram and muscle force. However, there exists instances where non-linear relationships were noted, as for example Kuroda et al. [50] who cited a linearity until maximum force is reached at which point the relationship between the averaged signal and the isometric femoral force sharply increases. Lawrence et al. [51] also cited a non-linear relationship for the bicep and deltoid muscle and a quasi-linearity for the first dorsal interosseous. 
The cited inconsistencies of the relationship between the electromyogram and isometric force have been attributed to several physiological and experimental effects [46]. MilnerBrown et al. [52] discussed possible explanations for the linearity of the relationship between force and the electromyogram, attributing to a constant number of active motor units and force induced by increased motor unit firing rates. The root mean square (RMS) of the electromyogram has also been noted by Milner-Brown et al. [52] to increase linearly to the squared root of the firing motor units. Physiological causes of the quasi-linear and non-linear observations of three muscles experimented on by Lawrence et al. [51] have included firing rate properties, crosstalk, and viscoelastic muscle properties.

The literature review in this section thus far has experimented with isometric contractions for different muscle groups; research into the relationship between force and isotonic contraction electromyograms has also been notable in the literature, as for example Arslan et al. [53] investigated the electromyogram force relationship recorded from the pectorialis major muscle under quasi-isotonic and isometric contractions in an effort to establish a new processing technique for electromyogram-force relationship analysis. Despite the wealth of literature relating to isometric and isotonic contraction signal and force relationship, the thesis's interest lies in the measurement of force during dynamic exercise. Nevertheless, such a relationship, as exhibited from the static contractions, does not exist with dynamic movement unless the task is restricted, as shown by Bigland et al. and Bouisset et al. [54].

Electromyography has previously been used in ergonomics to measure muscle activity and effort for static mouse tasks carried out by Agarabi et al. [55]. Their investigation assessed the design of four variations of a computer mouse: two standard designs, a slanted mouse and an ergonomic mouse, whilst measuring the activity from seven muscles that had previously been documented to be active during manipulation of a mouse. Agarabi et al. [55] concluded that the applied method for measuring muscle activity could be objectively utilized for the assessment of non-keyboard devices in the field of ergonomics. For gesture interactions, physical strain and effort was assessed by Riemer et al. [56]. The data collected from six muscles whilst performing eight gestures was analyzed by calculating the RMS and such calculation has shown the different gestures required varying degrees of effort. Riemer et al. [56] concluded that careful consideration of the physiological activity of muscles during hand gestures should be applied for interactive hand gesture selection.

\subsubsection{Fatigue}

In 1909 and 1912, Piper [57] was the first to notice the decrease in the electrical current as the muscle fatigued during a static contraction. Subjects were instructed to maintain their grip on a dynamometer for as long as possible while the electrical currents of the forearm muscles were observed using an electromyograph. An increase of signal amplitude was later noted by Cobb et al. [57] during isotonic and isometric contractions of the flexor carpi radialis, a muscle contributing to wrist flexion. Due to imperfect instrumentation, the work of Piper and Cobb et al. [57] remained in the lab. It was not until the 1950-60s that the experimental results systematically proved the influence of fatigue on the myoelectric signal recorded by an electromyograph. In 1951, Knowlton et al. [58] observed the increase of the signal's amplitude as the muscle fatigued.

There have also been instances reported of amplitude decrease during a fatiguing contraction [59]. Such unstable changes to the amplitude have lead scientists to conclude amplitude changes unreliable for the measurement of fatigue [60]. In 1977, Lindstrom et al. [61] developed a new method for the quantification of localized muscular fatigue based on the analysis of the power spectrum to provide information of the excitation of the muscle fiber's membrane. Lindstrom et al. [62] also concluded that the decrease in frequency and increase in amplitude are of the same physiological origin due to the excitation of the 
membrane; however, the usefulness of the power and time spectrum are not equal and the spectral analysis due to their stability through force changes.

Kwatny et al. [62] explored the power spectral density of electromyograms captured from the flexor pollicis brevis (thumb extension) and the extensor digitorum (fingers extension). Their experimentation method recorded electromyograms during isometric contractions, the signal was later digitally processed and the mean power frequency (MPF) and averaged amount of variability were computed before and during fatiguing contractions. Stulen et al. [63] have proved the median power frequency (also with the acronym MPF) to be reliable and consistent in the estimation of localized muscular fatigue due to muscle fiber conduction velocity. Zero crossing is another measure of frequency that is similar to the median and mean power frequencies [64][58].

Despite the majority of the aforementioned studies analyzing electromyograms for fatigue indexing during static contractions, fatigability in a natural setting is most often caused by dynamic exercise [65]. Bonato et al. [58] processed electromyograms, recorded during dynamic contractions, using six different distributions, based on experimentation ChoiWilliam transform of the time-frequency distribution was suggested as the most suitable compared to five other distributions. A later study carried out by Karlsson et al. [66] compared the application of Choi-William, continuous wavelet, Wigner-Ville and shorttime Fourier transforms finding the continuous wavelet transform to be more accurate in the detection of fatigue in dynamic exercise. Nevertheless, dynamic exercise poses complication for fatigue indexing compared to static contractions, as demonstrated by the relative movement of the electrode and the questionable assessment of electromyograms obtained from that electrode [67][68].

In the human-computer interaction discipline, surface electromyography has been used to assess the fatigability of interaction techniques [58][69][70] and software [59][71]. Murata et al. [72] evaluated the shoulder's muscular fatigue during video display terminal (VDT) tasks and assessed several techniques appropriated for biomechanics and ergonomic research. Electromyograms were recorded during isometric contractions collected before and at the end of four tasks. The signals were processed in the time and frequency domain using RMS and MPF (mean) respectively. Of the two processing techniques MPF proved more sensitive to localized fatigue. In contrast, a similar experiment carried out by Niu et al. [70] did not show any significant changes to the processed signal of isometric contraction and sampled recording during VDT tasks. For multi-touch interaction techniques, Lozano et al. [59] assessed the fatigability of a series of multi-touch gestures on a tablet in different conditions. The signals obtained during the gestures were processed using RMS and evaluated as a percentage of MVICs collected pre- and post-gesture tests. Lozano et al. [69] concluded that multi-finger gestures result in greater muscle activation and therefore encouraged the careful design of multi-touch technology for safer practice.

\subsection{General Discussion and Conclusion}

In this chapter, techniques for the assessment of muscle activity and fatigue have been briefly described and assessed for application in this thesis. Electromyography was chosen as the objective measure of muscle activity and fatigue in this thesis; an early selection has been made due to the invasiveness of some popular alternative techniques or a professional clinical requirement for application. A wealth of research can been found on the applicability of the electromyograph in various fields and tasks. In biomechanics, the relationship between isometric force and an electromyogram has been noted to be linear by Lippold [48], Close [49], and Kuroda et al. [50], where as Lawrence et al. [51] reported on a nonlinear relationship. The discrepancy of the results has been attributed to physiological and experimental effects, as stated by Milner-Brown et al. [52] and Lawrence et al. [51]. The popular processing techniques for extracting force information from the raw 
electromyogram are the integration of a rectified electromyogram [48][51] and RMS [50][52][55] of a signal. Electromyography has also been employed to index fatigue. A noted shift in the power spectrum to the left during a fatiguing contraction has been reported by Piper (via [57]), Kwatny et al. [63], Stulen et al. [64], and Murata et al. [70]. In the time domain, the amplitude has been noted to increase by Cobb et al. [57], Knowlton et al. [58], Murata et al. [70], and Lozano et al. [69], and to decrease by Niu et al. [59]. Due to this discrepancy in the analysis of fatigue in the time domain, the median power frequency of isometric contractions has been adopted for this thesis.

The next chapter considers techniques for the subjective assessment of physical workload for application in this thesis, in which the overall tabletop interaction task is assessed for overall fatigability over an extended period of time. 


\section{Chapter 4}

\section{Subjective Physical Workload Assessment}

\subsection{Introduction}

In this chapter, a review of prior research that is relevant to the subjective measurement of fatigue is given. Section 4.2 provides an outline of prominent subjective measures used for workload assessment. The subsequent subsections selectively assess the reviewed workload measure for use in this thesis, listing potential for application and limitations where admissible. The chapter then concludes in Section 4.3.

\subsection{Subjective Physical Workload Measures}

Subjective ratings are commonly used in the field of ergonomics to measure mental and physical workload, usability, fatigue, and many other aspects that can be quantifiably perceived. DiDomenico et al. [73] define subjective workload measures as " [an] attempt to quantify the effort exerted during task performance, using numerical ratings that do not directly measure either task performance or physiological responses to work." Hence, subjective measures are based on a person's self-perception and judgment of their exerted effort when performing a task or a combination of tasks. Even though objective measures are inherently preferred over their subjective counterpart in most fields, they are often adopted to supplement the objective assessment. The appeal of subjective measures lies in their practical administration, ease of use, simplicity, and their flexible adaptation to the differences between people and their abilities. Nevertheless, Annett [74] assessed the limitation of subjectivity for scientific investigation and reports on the influence of the goals, motives, and intentions of the assessed individual on the reported score.

The following subsections evaluate three of the most commonly used subjective physical workload measures in literature: visual analog scales, Borg scales, and NASA task load index $($ TLX). For each subsection, the measure is briefly explained. This is then followed by a survey of prominent applications for that measure. Lastly, the benefits and limitations of each measure are assessed in their ability to aid the achievement of the thesis goal.

\subsubsection{Visual Analog Scales}

Visual Analog Scales (VAS) are subjective instruments for used to measure a characteristic levels on a scale of none to extreme. The validity of the scale for chronic and experimental pain was investigated by Price et al. [75] and was found to be simple and has enabled easier responses as well as proving to be reliable for the assessment of the magnitude and intensity of both categories of pain examined. Psychological researchers have extensively adopted VAS as mood scales, for example Zealley et al. [76] found the scale to be effective and reliable for the assessment of depressed patients moods. VAS application has also been noted in human computer interaction research for technological ergonomic [77] and usability [78] evaluation. Furthermore, VAS, as the continuous assessment scale of choice for Belz et al. [79], proved as beneficial as the lone Neuro-linguistic programming (NLP) 
subjective scale and Verbal Descriptor Scales (VDS), with NLP raters favoring VAS over VDS in the study.

\subsubsection{Borg Scales}

An individual's perception of a task's exertion level is a good indication of the level of physical strain [80]. The Borg scales refer to a set of three perceived exertion scales developed by Gunnar Borg: Rating of Perceived Exertion (RPE) [81][82], Category Ratio 10 scale (CR10) [82], and a Category Ratio 100 scale “centiMax" (CR100) [83]. Borg's RPE scale is a 15 -grade verbally anchored scale ranging from 6 to 20 , where the starting point is indicative of the lowest heart rate estimate for adults and the last point represents an unlikely level of exertion to ever occur in most people. In 1982, Borg developed CR10 to meet the demands of supplying a ratio scaling method that directly estimates the level of exertion and also determines the ratio relationship between perceptual responses [82]. The ratio category scale is composed of verbally anchored scales ranging from 1 to 10 , however, unlike the RPE scale there is no ceiling value and decimal scores can be used to describe the level of exertion perceived. CR100 was later developed as a finer graded scale compared to CR10, which contains subjective dynamic ranges with values ranging from 0 to 120 annotated with verbal anchors [83]. Similar to the CR10 scale, the structure encouraged decimal ratings with no upper limit.

In human-computer interaction, the Borg scales have been applied to quantitatively evaluate mobile augmented reality [84][85], wearable systems [86], and software ergonomics [71][72]. Arvanitis et al. [85] applied Borg's RPE scale to evaluate the application of augmented reality in science education. The mobile augmented reality system, CONNECT, aimed to support physically disabled learners; its use was evaluated for perceived exertion and localized discomfort and/or pain for both able-bodied and disabled learners. The mobile system proved to be laborious for both learners based on data obtained using the RPE scale. For the evaluation of wearable systems, Weller et al. [86] assessed the use of a wearable system designed for use in intensive care units (ICU). With the use of existing scales, RPE, CR10, and Rapid Entire Body Assessment (REBS), Knight et al. [84] developed a methodology for determining the wearability of a mobile augmented system which was used by Weller el al. [86]. The results of the methodology carried out on the ICU wearable system found it to be wearable and suitable for real-time patient status information update.

Comparison between Borg scales and VAS, briefly described in subsection 4.2.1, have been carried out by Neely et al. [87], Grant [88], and Capodagilo [89] for the evaluation of leg exertion, treadmill exercise, and arm-cranking exercise respectively. Neely et al. [87] reported the high correlation between CR10 and VAS, and stated that the advantage of CR10 over VAS lies in its differential perception between very extreme and maximal forces. During a treadmill exercise, Grant [88] assessed the sensitivity of three subjective scales: VAS, CR10, and a Likert scale (LS) to breathlessness and general fatigue. Of the three, VAS and LS proved more sensitive to the measure of breathlessness, whereas CR10 proved significantly sensitive to the overall fatigability of the task. Grant concluded that the VAS is the best scale for steady-state exercises. Conversely, Capodagilo [89] found CR10 as positively sensitive to breathlessness, as linear as VAS was in the arm-cranking exercise. The CR10 scale was also found to be responsive to two physiological variables: heart rate and blood lactate.

A comparative study of Borg's three scales was carried out on a bicycle ergometer test by Borg et al. [90] in two experiments: the first compared Borg's RPE and CR10 scales and the second compared the three scales. The results of the first study have shown that the heart rate measures collected during the task highly correlated with the RPE scale, whereas the CR10 correlated with both physiological variables collected for the heart rate and blood lactate. The comparison carried out between CR10 and CR100 did not show a significant 
difference between the two scales. Borg et al. [90] concluded that the benefits of the RPE scale lie in its simplicity and ease of use but points out the advantages of applying psychophysical scales in the area of perceived exertions. In spite of insignificant differences between the CR scales, the association between the CR100 scale and a percentage scales with finer graded ranges can prove beneficial for measuring perceived exertion.

\subsubsection{NASA TLX}

The NASA TLX is a multidimensional scale that is used to measure mental workload. It consists of six dimensions: mental demand, physical demand, temporal demand, performance, effort, and frustration, each with a twenty step scale with annotated end points ranging from "low" to "high". A global score is obtained by combining weighted averages of the scores from each dimension [91]; the weighted averages are specific to the user's input of the dimension's relevance. The NASA TLX scale has been applied in various human-machine environments, for example, in the evaluation of simulated flights [92] or driving performances [93]; in the human-computer interaction discipline it has been used to evaluate assistive technology [94], ubiquitous technology [95], software ergonomics [71][72], and interaction techniques [96][97].

Due to its germaneness to the subjective measure selection to achieve the thesis goal, NASA TLX application for the evaluation of interaction techniques is briefly described. Shoemaker et al. [96] assessed a mid-air text input technique for very large vertical displays. Three techniques, whereby a handheld device is manipulated in mid-air, were developed: Circle, QWERTY, and Cube. Data from a NASA TLX based questionnaire assessing physical and mental workload were collected for each technique. The results proved that the QWERTY keyboard setting was the easiest in comparison with the Circle and Cube techniques. Subsequently Pedersen et al. [97] evaluated a set of interaction techniques that utilized active motorized tangibles on a tabletop, also known as tangible bots; the evaluated techniques included haptic feedback, indirect and imitative tangible movement and rotation, and group interactions. A set of tasks, employing active and passive tangible bots, were evaluated using the NASA TLX to measure perceived subjective mental and physical task loads where active tangible bots proved superior to passive tangibles.

The benefits of NASA TLX are well established in comparative literature. In 1985, Vidulich et al. [98] found NASA TLX had a smaller between-subject variability compared to the Subjective Workload Assessment Technique (SWAT). Hill et al. [99] later carried out a similar comparison between NASA TLX, SWAT, and Overall Workload (OW); the sensitivity of NASA TLX and OW compared to SWAT when measuring blood lactate blood lactate was evidenced to be superior. Rubio et al. [100] compared three subjective measures, one of which was NASA TLX, and found that its scores have a better correlation with performance compared to Workload Profile Methods and SWAT. Nevertheless, one major concern in this thesis is the applicability of NASA TLX for sole physical workload assessment, despite similar published use [71][72].

\subsection{General Discussion and Conclusion}

Section 4.2 gave a brief overview of three subjective measures that have been popularly grounded in various disciplines such as psychology, ergonomics, and human-computer interaction. Due to their simplicity and supplementary status for this thesis, two unidimensional scales as opposed to one multidimensional scale have been assessed. The first unidimensional scale VAS is appealing due to its versatile application, as it is possible to scale any form of characteristic using VAS, and its simplicity. Price et al. [75] and Zealley et al. [76] have recognized the simplicity of VAS in its application for the subjective assessment of pain and mood respectively. The second surveyed unidimensional scales, the Borg scales, are designed to give precise reflections of subjectively perceived exertion during any form of dynamic or static exercise. Borg's RPE and CR10 scales have been extensively applied to prior experimentation, for example in human-computer interaction it 
has been applied for the subjective evaluation of hardware [85] and software [72]. Comparisons between the unidimensional scales surveyed: the two Borg scale, RPE and CR10, and VAS, have been carried out by Neely et al. [87], Grant [88], and Capodaglio [89]. All three studies have acknowledged the superiority of the Borg scales over the VAS scales as they have positively correlated with physiological responses to the dynamic exercises used for evaluation.

The third scale reviewed was NASA TLX, a multidimensional scale for the assessment of overall workload. Unlike the other two scales reviewed above, NASA TLX takes into consideration mental demands, frustration, effort, performance, and temporal demand in addition to physical demand. The focus of this thesis is to assess the physical demand and the fatigability of tabletop interaction techniques, thus the application of NASA TLX could be excessive when considering the goal of the thesis. It should be noted that instances of NASA TLX adoption for evaluation has been found in prior literature [71][72]. Due to the outperformance of Borg scales over VAS and the complexity and excessive application of NASA TLX, in this thesis, the selection of the Borg scales proved preliminarily suitable.

Section 4.2.2 reviewed Borg's scales: RPE, CR10, and CR100. In accordance to our thesis goal, the Borg scales were inherently used to quantify perceived exertion. The application of the RPE and CR10 scales is well documented in literature, for example in human-computer interaction RPE was used to subjectively evaluate augmented reality systems $[84]$ and to develop, in combination with CR10 and REBS, a methodology for the assessment of a wearable system's wearability $[86]$. Of the three scales, the CR scales outperformed the RPE scale in a dynamic evaluative exercise carried out by Borg et al. [90], however, no significant differences between the CR scales were noted. In spite of insignificant differences between the CR scales, the association between the CR100 scale and a percentage scale with finer graded ranges can prove beneficial for the measurement of the level of perceived exertion. A second appeal in its application in this thesis is its novel application for the evaluation of interaction techniques.

Chapter 5 now moves on to account for the materials and methodologies applied in this thesis for the assessment of tabletop interaction techniques and their fatigability over an extended period of time. 


\section{Chapter 5}

\section{Experimental Methodology and Materials}

\subsection{Introduction}

This chapter describes and explains the methods and materials employed for the achievement of the thesis goal. Firstly, the experiments' rationale and goals are further elaborated from Chapter 1. Section 5.2 describes the apparatuses and materials used, when applicable details of implementation are also given. The section also states subjective instrumentation used in the experiments. This is followed by the necessary details of the subjects participating in both experiments. In Section 5.4 the techniques used to conduct both experiments are elaborated. The analysis techniques used for the assessment of the results of the experiments are listed and described in Section 5.5. The chapter then concludes with an overall summary in Section 5.6.

\subsubsection{Experiments Rationale}

It was in 1945 that Bush [1] envisioned an interactive electromechanical device for the manipulation of records, which he named memex. His vision was later pioneered by Wellner [2] with the implementation of DigitalDesk; the technology is now familiarly known as tabletops to signify the location of the display. It was also noted by Saffer [7], that DigitalDesk was the first multi-touch interactive device that used some of today's familiar interactive gestures, such as pinch to shrink and spread to expand. Despite early attempts to introduce multi-touch technology, at a commercial level, with phones [10] and tablets [31], dating back to 1992, its diffusion into people's every day lives was marked by the introduction of the iPhone and iPod touch in 2007. In the same year, Microsoft unveiled their multi-touch tabletop, MS Surface. Interacting using touch-screen phones, tablets and tables are proving very popular in the modern world and have yet to lose their novel appeal.

Bimanualism, the act of using both hands to perform a task, is a naturally intuitive behavior adopted in various tasks. For example, writing on paper by positioning the paper with the non-dominant hand while writing with the dominant one, and throughout the writing task the non-dominant hand continues setting a frame of reference for the dominant hand to refer to when performing its assigned task. The countless benefits of bimanual interaction techniques, both symmetrical and asymmetrical, have included but are not limited to: adaption of existing skills [23][26][28], parallelism [21][32], and outperformance of bimanual techniques over unimanual ones as the difficulty of the task increases [31][33].

Most studies carried out for the evaluation of interaction techniques have addressed the benefits of one technique over another at certain tasks or traditional and novel interaction techniques. For example, the benefits of bimanual interactions over unimanual ones or the benefits of direct touch over traditional human-computer interactive technologies [17][18]. Investigation into the ergonomics of traditional techniques has also been noted in the literature [59][70-72][77]. However, only a few addressed the ergonomics and fatigability of multi-touch gestural interactions [69][56]. The novelty of those techniques, despite its appealing portrayal in science fiction literature and movies, and its effect on the 
musculoskeletal system must be addressed in order to facilitate healthier everyday adoption.

\subsubsection{Experimental Goal}

The studies were performed with three goals in mind. The first goal was to understand user's preference using natural interaction techniques over an extended period of time on horizontal and vertical tabletops in a normal work setting. Secondly, to further understand that preference by objectively measuring physiological changes to the muscles prior to the hour-long interactive task and at the end of each condition's task. The comparisons carried out between the pre- and post-recordings should be indicative of the fatigability of the task on the three muscles assessed on each arm, a similar technique was carried by Murata et al. [70] and Niu et al. [59]. A further addition to the second goal is to measure the electrical activity of each muscle at 5-minute intervals during the evaluation task. This technique should clarify which muscles are being used, how extensively the muscles are being used, and whether they are being used unimanually or bimanually. The third and final goal was to collect subjective ratings of the overall perceived exertion from the interactive task on the horizontal and vertical tabletop.

\subsection{Materials and Instruments}

The following subsections discuss and describe the materials and instruments employed in this thesis. The apparatus used are described in Section 5.2.1 this is then followed by the instrument adopted. Section 5.2.3 elaborated on the task adapted to experiment's requirements. Finally, the experimental setup for both horizontal and vertical conditions is portrayed in Section 5.2.4.

\subsubsection{Apparatus}

The tabletop of choice was Microsoft's Surface, a vision-based, multi-touch surface with a 30 -inch rear-projected display at $1024 \mathrm{X} 768$ resolution that is 22 inches high, 21 inches deep, and 42 inches wide. The custom software running is Windows Vista with Ethernet, wireless, and Bluetooth connectivity. The tabletop is acrylic with an interior powder-coated steel frame [101]. Finger and object contacts with the display surface are tracked with diffused infrared lights, which also track $2 \mathrm{D}$ visual markers. The experimental task developed for the MS Surface used Microsoft Surface SDK for input and XNA library for graphic rendering.

The electromyograph used for the experiments was ZeroWire, a wireless electromyograph system with 8 surface channels. It operates using light autonomous signal processing and power transmission units, each weight 10 grams. Each channel provides a bandwidth of 10-1000 $\mathrm{Hz}$ for a signal sampled at 2000 sample/sec [102]. The wireless technology enables the acquisition of electromyograms without restricting normal interaction. The electrodes used with the ZeroWire electromyograph were Biosense's biologic disposable press-stud electrodes $(\mathrm{Ag}-\mathrm{AgCl})$. The transmitters, through $\mathrm{WiFi}$, transfer the signals captured with the electrodes to the main unit, which is directly connected to a MacBook running the ZeroWire software. Matlab and Microsoft Excel were later used to process the electromyograms and statistically analyze the results respectively. 


\subsubsection{Subjective Instrument}

Subjective ratings are commonly used as supplements to objective measures to assess perceived physical exertion in various disciplines. For the purpose of the experiments, VAS, Borg's scales, and NASA TLX have been critically assessed for application.

VAS ratings have been used to assess pain [75], depressive mood swings [76], technical evaluation [77], usability assessment [78], and NLP [79]. The obvious appeal of VAS is its simple and versatile application where the scores reported were valued between two extremes of a characteristic. Price et al. [75] reported that VAS enticed simpler responses for the subjective assessment of pain and its reliability has been proven by a positive correlation with physiological pain measures. A similar report is noted by Zealley et al. [76] that found the scale to be effective and reliable for the assessment of depressed patients moods. Despite its reported simplicity and reliability for the assessment of pain and mood swings, VAS was negatively compared against Borg's CR10 scale in various dynamic exercises [87-89]. Borg's CR10 is a category ratio scale designed to meet the demands of supplying a rating method that directly estimates the level of exertion and the ratio relationship between perceptual responses; both demands have not been met in Borg's previous scale, RPE [82]. As previously stated, Borg's CR10 outperformed VAS as the ratings were compared against physiological responses and the attributed benefits were: differential perception between very extreme and maximal forces [87] and positive correlation to breathlessness [89] and blood lactate [88].

The outperformance of Borg's scale over VAS led to preliminarily selecting Borg's scales for possible application in this thesis. A second survey carried out between NASA TLX and Borg's scales was required. NASA TLX has been chosen as the assessment technique for overall workload, including both physical and mental workload among other dimensions, listed in section 4.2.3. Nevertheless, NASA TLX was critically assessed as being complex [91] and excessive for application in this thesis. A decision to use Borg's scale lead to further investigation into Borg's three scales: RPE, CR10, and CR100. A comparative study carried out by Borg et al. [90] has found the CR10 to have a better correlation with physiological exertion responses, whereas RPE correlated breathlessness alone. No differences were noted between CR10 and CR100; however, CR100 had a better association with the percentage scale with finer graded scores for the assessment of perceived exertion. On the basis of the above analysis, Borg's CR100 was the subjective instrument used for the experiments.

\subsubsection{Task}

For the experimental task design, three conditions had to be fulfilled. Firstly, the task should reflect practical interactions and interaction techniques on tabletops. This included gestures for bimanual and unimanual rotation, scaling, and translation. Secondly, the task should be engaging and be able to induce almost constant interactions with the tabletop. Thirdly, the task should be easily adapted for use on horizontal and vertical tabletops.

ScatterPuzzle is an MS Surface application that implements ScatterView and ScatterViewItem to create a puzzle. The sample application is part of the Surface toolkit for Windows touch beta, where the source code can be obtained [103]. ScatterView and ScatterViewItem are control classes that encapsulate and bind an item to enable any type of manipulation of that item on the touch display; the manipulation included rotation, scaling, and translation that can be set to true or false for customization. In the case of ScatterPuzzle, the controls encapsulate geometric images representing the different puzzle pieces and allowed the rotation and translation of those pieces across the display. Two other controls, SurfaceListBox and SurfaceSlider, were implemented in ScatterPuzzle that allowed the selection of an image to be used as a puzzle for a list box and the selection of a difficulty level to control the number of puzzle pieces for a selected image. Figure 5-1 left 
panel) shows a run-time illustration of ScatterPuzzle. Thus far, ScatterPuzzle fulfilled the second and third conditions, and two thirds of the first condition. Nevertheless, since ScatterViewItems and ScatterView can be set to be scaled integrally, ScatterPuzzle was adopted for this thesis's experimental task was made.
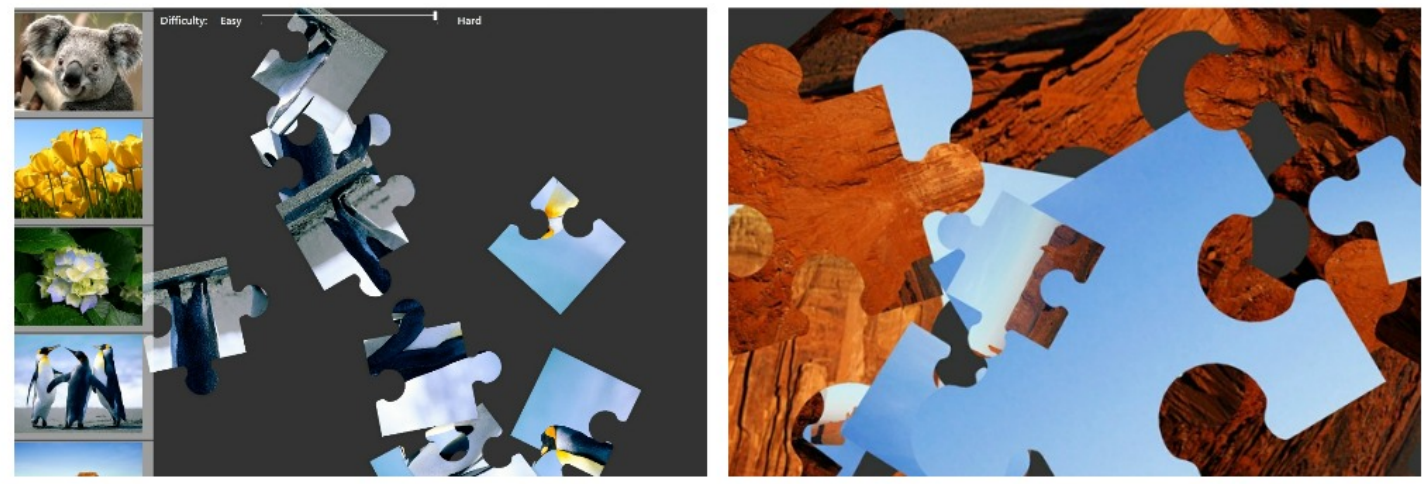

Figure 5-1: the right panel shows the ScatterPuzzle developed by Microsoft and left panel shows the modified application used in the study

The main concern with the ScatterPuzzle application was the inability to scale the pieces; therefore, the first step was to enable the scaling of the pieces. The purpose of providing larger and smaller pieces was to entice bimanual interaction for larger pieces for which multiple unimanual gestures would otherwise have to be employed. In the original ScatterPuzzle, all pieces were of the same scale and are merged into one if two pieces matched. Despite enabling the scaling of individual pieces, once two pieces matched, even if they were different scales, those pieces merged into one. This behavior proved unsuitable for the experimental task, since a user might disregard scaling if a match can be made with different sized pieces. Additional changes were made to overcome this problem and to allow only approximately same-sized pieces to be merged into one, thus requiring scaling manipulations to match the pieces.

A SurfaceSlider was used to determine the level of difficulty of the puzzle, i.e. number of pieces; the number of pieces ranged from 4 to 9 . To make the task more difficult with longer engagement times, the number of puzzle pieces was increased to 25 . It was hypothesized that as the difficulty of the task increases, the more likely it is that more manipulation will ensue in attempts to find the matching pieces. The choice to remove the SurfaceSlider was also made to standardize the task for all participants. It was for the same reason that the SurfaceListBox was removed and replaced by a static number of puzzles with a fixed order so that once a puzzle is completed another replaces it. The adapted ScatterPuzzle is shown in Figure 5-1 (right panel).

The experimental task now involved the manipulation of 25 puzzle pieces to construct an image. The manipulation of the individual pieces included: pinch to shrink and spread to expand using two or more fingers from one or two hands, rotate using two or more fingers from one hand or two hands, translating using one or more fingers using one or two hands, and an integral rotate, scale, and translate manipulation using one or more fingers from one or two hands. Two matching pieces can only connect to form one larger piece if they are approximately the same size and orientation with a threshold of 0.2 and $30^{\circ}$ respectively. On completion the puzzle is then replaced with another until the allotted experimental time is over.

\subsubsection{Experimental Setup}

For the horizontal condition, the MS Surface did not prove ideal at 22 inches, thus it was heightened to 26 inches using four, approximately 1 inch long wood panels, raising it to the reported ideal table top height for chairs at an 18-inch height. The purpose was to set up 
the tabletop comfortably in a way that allows the legs of the participant to slide under the tabletop display. Nevertheless, this was not completely possible since the MS Surface has a bulky frame beneath the display. In the second experiment, the MS Surface was vertically propped up vertically on top of a work desk. Both experimental setups are shown in Figure 5 -2. Both experiments were conducted while being seated on a chair of 18 inches height.
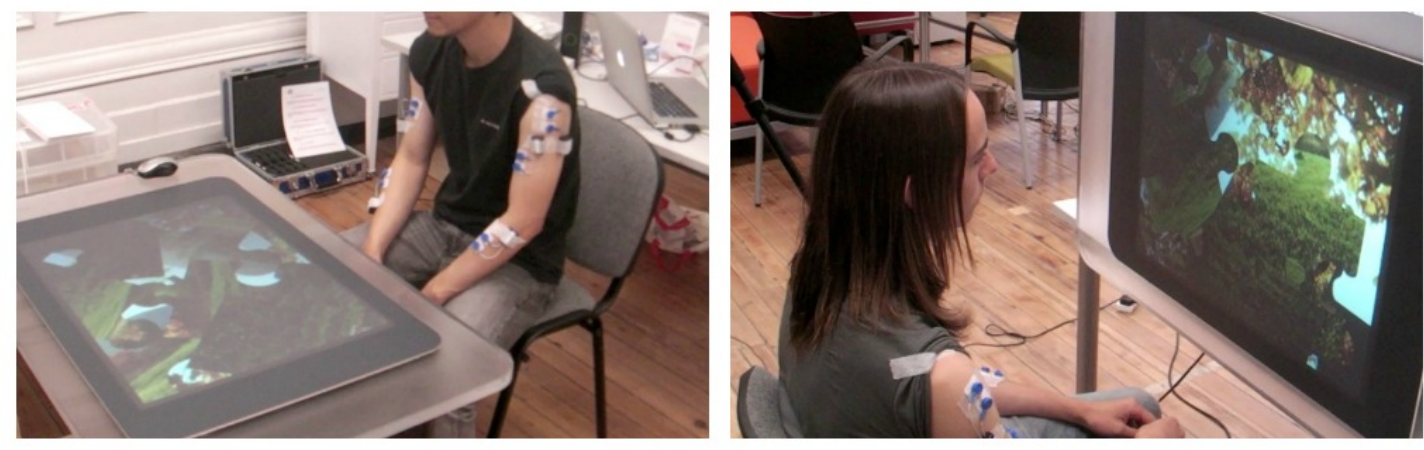

Figure 5-2: the experimental setup for the horizontal (right) and the vertical (left) conditions

\subsection{Participants}

The first experiment, documented in Chapter 6, was carried out with the participation of six subjects (5 males and 1 female), however, the data from two of the six muscles measured for one of the subjects was lost due to technical difficulties and thus the data from that subject is not reported here. The mean age of the five participants $(4$ males and 1 female) from whom data was obtained is $26 \pm 5$.4, all of which reported verbally to be right-handed and free of musculoskeletal disorder in their upper extremities. Although, all subjects reported to be familiar with multi-touch technology, only one has worked with the MS Surface before. For the second experiment, reported in Chapter 7, six participants 5 males and 1 female) volunteered with an average age of $25.3 \pm 5.9$ of whom none reported musculoskeletal disorder. Similar to the first experiment, all subjects were right handed with no prior experience with the MS surface but familiar with multi-touch technology. All subjects from both experiments have verbally consented to being filmed during the task and to have the areas where the electrodes will be placed to be shaved, if necessary.

\subsection{Procedure}

The three muscular activities recorded for each experiment from each arm were the middle deltoid, biceps brachium, and extensor digitorum. Lozano et al. [69] observed those muscles for measuring the fatigability of gestural interactions on a multi-touch tablet while also using an electromyograph. A review of those muscle actions proved suitable for adoption in this thesis, with slight alteration to the deltoid placement from anterior to middle so as to consider shoulder abduction during the vertical condition and at the same time still be able to measure shoulder flexion.

The middle deltoid muscle is a specific placement that detects a signal once the arm is abducted or flexed. The location of the muscle was detected by palpating the lateral aspect of the upper arm as the participants abducted their arms. The bicep brachium specific placement was detected by palpating the flexed bicep in the dorsal aspect of the upper arm, which is used to detect forearm flexion, forearm supination, and shoulder flexion. The placement of the extensor digitorum is quasi-specific, which means the muscle lies in close proximity to other muscles with a possibility of crosstalk from those other muscles. While palpating halfway through the forearm as the participants extended their fingers, the 
placement of the extensor digitorum was located for monitoring muscle activity of finger extension. Once the location was determined for each muscle, the skin was shaved and wiped with alcohol, thus eliminating possible interference to the signal. Then, the electrodes were placed parallel to their prospective muscles and $2 \mathrm{~cm}$ apart. The placement of the electrodes and parallelism for all three muscles was achieved by following guidelines provided by Criswell [45] and are shown in Figure 5-3.
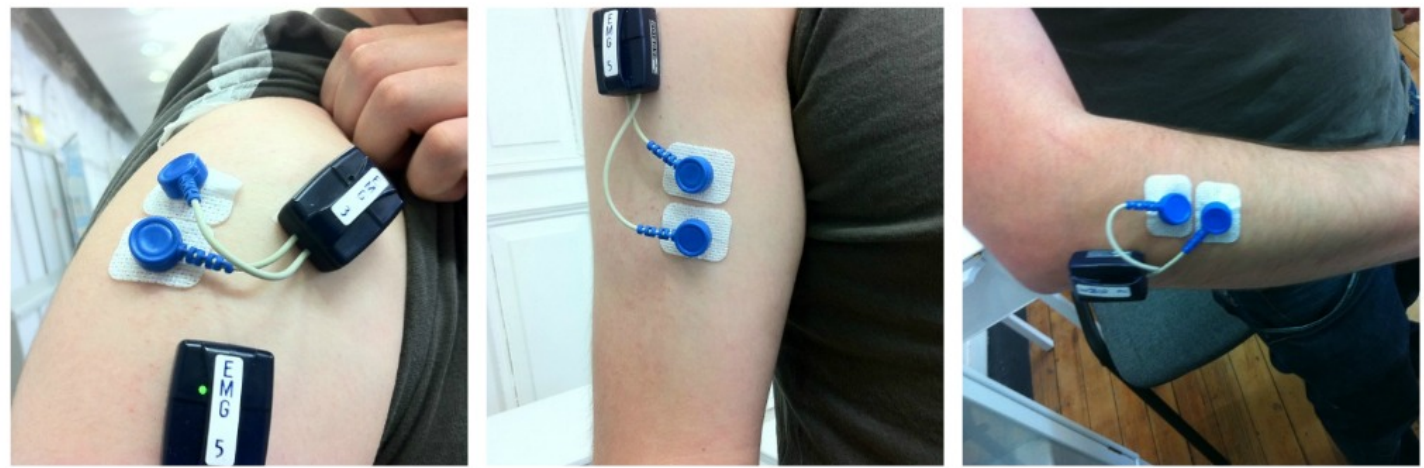

Figure 5-3: the placements of the electrodes for the middle deltoid (right), biceps brachii (middle), and the extensor digitorum (left) muscles

The first electromyograms recorded were achieved by asking participants to maintain a certain posture for each specific muscle so that the signal obtained is maintained under the same isometric contraction. For the middle deltoid muscles, participants were asked to elevate their arms at $90^{\circ}$ abduction in the frontal plane for 10 seconds while holding on to a $2.5 \mathrm{~kg}$ kettlebell (Figure 5-4-left panel illustrates the posture using a hanging weight for the right arm's middle deltoid). The isometric contraction for the biceps brachium was captured by asking the participants to keep that arm close to the body while elevating the forearm at $90^{\circ}$ abduction in the sagittal plane while holding on to a $2.5 \mathrm{~kg}$ kettlebell (Figure 5-4-middle panel shows the posture performed for the right biceps brachium). For the extensor digitorum muscle, participants were asked to place their forearm on a desk with the wrist resting on the edge of the table and holding on a $2.5 \mathrm{~kg}$ kettlebell (Figure 5-4-right panel shows the posture performed for the right extensor digitorum). All postures were performed while the participants were seated.
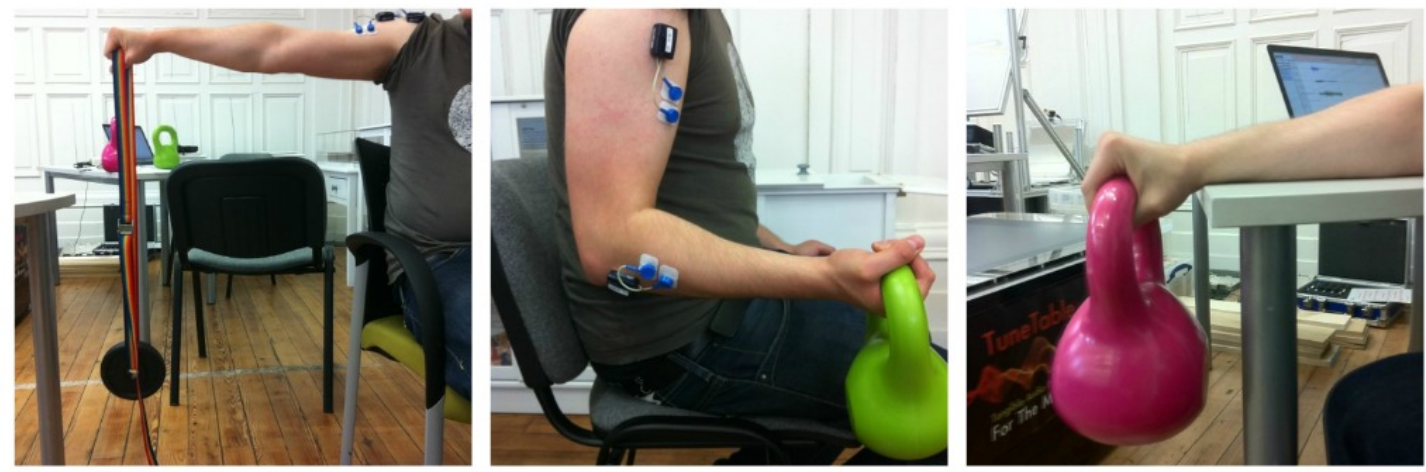

Figure 5-4: adopted postures to record isometric contractions for the middle deltoid (left), biceps brachii (middle), and the extensor digitorum (right) muscles

After the isometric contractions were collected, participants were asked to report their overall perceived exertion using the Borg CR100 scale; a brief description of the scale was also provided for reference. The scores were collected before and after the hour-long task. The participants were then asked to seat themselves comfortably at the tabletop and to familiarize themselves with the task for two minutes as the list of possible manipulations were stated verbally. After this, they were asked to wait for the start and the end of the 
session to be announced. During the session, ten electromyograms were recorded for muscle activity analysis.

\subsection{Data Analysis}

Four different datasets are obtained from each session, each with their own analysis requirements. The following subsections describe in detail the analysis method applied to the subjective ratings, isometric contractions, electromyograms, and film records.

\subsubsection{Subjective Ratings Analysis}

The first dataset to be analyzed compared the average of both scores collected before and after the experimental task. To signify the results statistically a paired t-test was carried out between the subjective overall perceived exertion ratings recorded before (set 1) and after (set 2) the experimental task. A null hypothesis was stated where

$$
H_{0}: \mu_{1}=\mu_{2}
$$

Also an alternative hypothesis was stated where it is believed that $\mu_{1}$ is smaller than $\mu_{2}$

$$
H_{1}: \mu_{1}<\mu_{2}
$$

The difference between the observations on each pair was calculated where $x$ is a value from set 1 and $y$ is a value from set 2

$$
d_{i}=y_{i}-x_{i}
$$

From which the mean difference is calculated $\mu_{d}$. Afterwards, the standard deviation $(\mathrm{STD})$ of the difference is calculated, where it is used to calculate the standard error (SE) of the mean difference where $n$ is the size of the set

$$
S E\left(\mu_{d}\right)=\frac{s_{d}}{\sqrt{n}}
$$

The t-statistic is then calculated using the following equation

$$
T=\frac{\mu_{d}}{S E\left(\mu_{d}\right)}
$$

Finally, under the null hypothesis the statistic follows a t-distribution with $n-1$ degrees of freedom, which is then looked up in the $t$-distribution table that gives a $p$-value determining the truthfulness of the null hypothesis.

\subsubsection{Isometric Contraction Electromyogram Analysis}

The second dataset to be analyzed is the isometric contractions collected before and after the experimental task. First, the 10-second electromyogram was divided into 10 segments with a $50 \%$ overlap that led to 3000 data points for each segment from an original 2000. The last segment was excluded from the analysis as a consequence of the overlap. Each segment was then rectified and passed through a low pass fourth-order Butterworth filter with a cutoff frequency of $500 \mathrm{~Hz}$. A fast Fourier transform was then performed to calculate the power spectrum of each segment from which the median power frequency is obtained. Matlab was used for the processing of the electromyogram with the set of functions provided by the Signal Processing Toolbox (DSP) and the Biomechanics et al. Toolbox (BEAT) [104]. The Matlab function employed for isometric contraction analysis can be found in Appendix A.1.

For normalization, the isometric contractions collected before the task were averaged and used as a reference value for the isometric contraction recorded after the task. This 
normalization procedure eliminates inter-individual variations between the participants. The normalized values were then averaged to produce singular values representing the median frequencies of each muscle.

A paired t-test was also carried out between the isometric contraction electromyograms recorded before (set 1) and after (set 2) where the null hypothesis stated that the means of the two sets were equal

$$
H_{0}: \mu_{1}=\mu_{2}
$$

And an alternative hypothesis that stated that the mean of set 1 is larger than set 2 , since the mean power frequency decreases with fatigue during an isometric contraction

$$
H_{1}: \mu_{1}>\mu_{2}
$$

The steps illustrated in Section 5.5.2 were followed to establish a p-value to determine the validity of the null hypothesis.

\subsubsection{Electromyogram Analysis}

Ten 1 minute electromyograms were collected during the experimental task at 5 minute intervals. Table 1 illustrates the time blocks and their designated time in the task. Each electromyogram was passed through a low-pass fourth-order Butterworth filter with a cutoff frequency of $500 \mathrm{~Hz}$. The averaged RMS was then calculated using the RMS method from the Biomechanics et al. Toolbox (BEAT) [104] which was then averaged. Similar to Section 5.5.2, normalization was carried out using the averaged isometric contraction collected before the start of the task. The normalized values were then averaged to represent the muscle activity as a percentage of the isometric contraction. The Matlab functions employed for isometric contraction analysis can be found in Appendix A.2.

\begin{tabular}{|c|c|}
\hline Time Block & Time in Task \\
\hline 1 & $4-5$ \\
\hline 2 & $10-11$ \\
\hline 3 & $16-17$ \\
\hline 4 & $22-23$ \\
\hline 5 & $28-29$ \\
\hline 6 & $34-35$ \\
\hline 7 & $40-41$ \\
\hline 8 & $46-47$ \\
\hline 9 & $52-53$ \\
\hline 10 & $58-59$ \\
\hline
\end{tabular}

Table 5-1: the time blocks and their designated time during task, all of a length of 1 minute

A one-way repeated measure analysis of variance (ANOVA) was later carried out on the processed data where the null hypothesis stated that the means of the ten sets were equal and an alternative hypothesis that states that at least one mean is different. The ANOVA test was carried out using Microsoft Excel's analysis tool pack.

\subsubsection{Video Analysis}

The experimental task was filmed for all participants. The films were analyzed and noted for any significant manipulation relevant to this thesis. The observations carried out included determining the techniques employed throughout the task, any changes to technique employment over time, and postural constraints of the tabletop setting. 


\subsection{Conclusion}

This chapter rationalized the experimental goals and described the materials and instruments employed in this thesis. The apparatuses used on evaluated tested setting and for objectively measuring muscle activation is listed in Section 5.2.1, Microsoft Surface and ZeroWire electromyograph respectively. The following section explained and rationalized the use of the selected subjective measure selections. Section 5.2.3 described the task used for evaluation, which included brief implementation details. This was followed by a description of the experimental set up for the two evaluated conditions. Section 5.3 gave details of the participants for the two tabletop conditions, this included age, gender, handedness, and upper extremity disorders. Section 5.4 illustrated and detailed the experimental procedure, which was then followed by the analysis employed for the assessment of the collected data in Section 5.5. 


\section{Chapter 6}

\section{Experiment I}

\subsection{Introduction}

The results of the experimental procedure carried out for the horizontal tabletop is described and later analyzed in this chapter. Section 6.2 identifies the purpose of this experiment and its relevance to the thesis goal. This is followed by a list of hypotheses of the expected results. In Section 6.4 the results are described in subsections corresponding to the dataset analyzed. Lastly, the chapter concludes by discussing the results of the experiment against the hypotheses while summarizing the findings.

\subsection{Purpose of the Experiment}

The purpose of the experiment is to get insight into the interaction techniques adopted during tabletop interactions for an extended period of time. Also, an ergonomic perspective is captured for interactive tabletop experience where the six muscles assessed are objectively analyzed for muscle activation during the experimental task and fatigability of interaction at the end of that task. With such materials better and less harmful interactions can be designed for upcoming technology with the true potential of traditional workstation substitution.

\subsection{Hypotheses of Expected Results}

The following are the hypotheses of the expected results for the datasets obtained from the experimental session for the horizontal tabletop condition. The hypothesis for the subject ratings collected before and after the experimental task is that the averaged values collected after the task will be greater than that of the averaged before values, thus indicating the fatigability of the tabletop interaction. Similar hypotheses are stated for the recorded isometric contraction of the six assessed muscles, where the MPF calculated for the contractions recorded after the task is smaller than the one captured and processed before the task, in turn proving the fatigability of that muscle. The hypothesis further states that fatigue is noted for the right (dominant side) extensor digitorum, middle deltoid, and biceps brachii, however none or less fatigue is noted for their left counterparts.

With respect to the recordings collected during the task at 5-minute intervals and the film analysis, it is hypothesized that as time progresses subjects will fall back on bimanual interaction techniques, as they are more convenient than unimanual techniques. The results of the film analysis should be evident in the recordings captured during the task, where a normalized activation is higher for the assessed right muscles than that of their left counterparts further along the task. 


\subsection{Results}

The following subsections review the results of the analysis of the four datasets described in Section 5.5: subjective ratings, isometric contraction electromyograms, electromyograms, and videos.

\subsubsection{Subjective Ratings Results}

The averaged values of the subjective ratings that were collected after the task proved higher than that of those collected before the task. Statistical significance was calculated using a paired t-test carried out between the two datasets of overall perceived exertion recorded for the horizontal condition. The results have shown, in regards to a null hypothesis that stated that the two means of the before and after sets are equal, that with $p=0.0773$, there exists slight evidence against the null hypothesis, but not enough to reject it. The detailed results are included in Appendix B.1.

\subsubsection{Isometric Contraction Electromyogram Results}

Isometric contraction were recorded before and after the experimental task, which was then processed by rectifying the signal and then passing it through a low-pass filter to remove traces of noise. The signal was then transformed from the time to the frequency domain from which MPF for each before and after recording was calculated. To overcome interindividual variations between the subjects, the data collected after the task was normalized as a percentage of the isometric contraction recorded prior to the task. Thus, each percentile is representative of the MPF of each recorded muscle contraction. The results of the analysis are found in Figure 6-1.

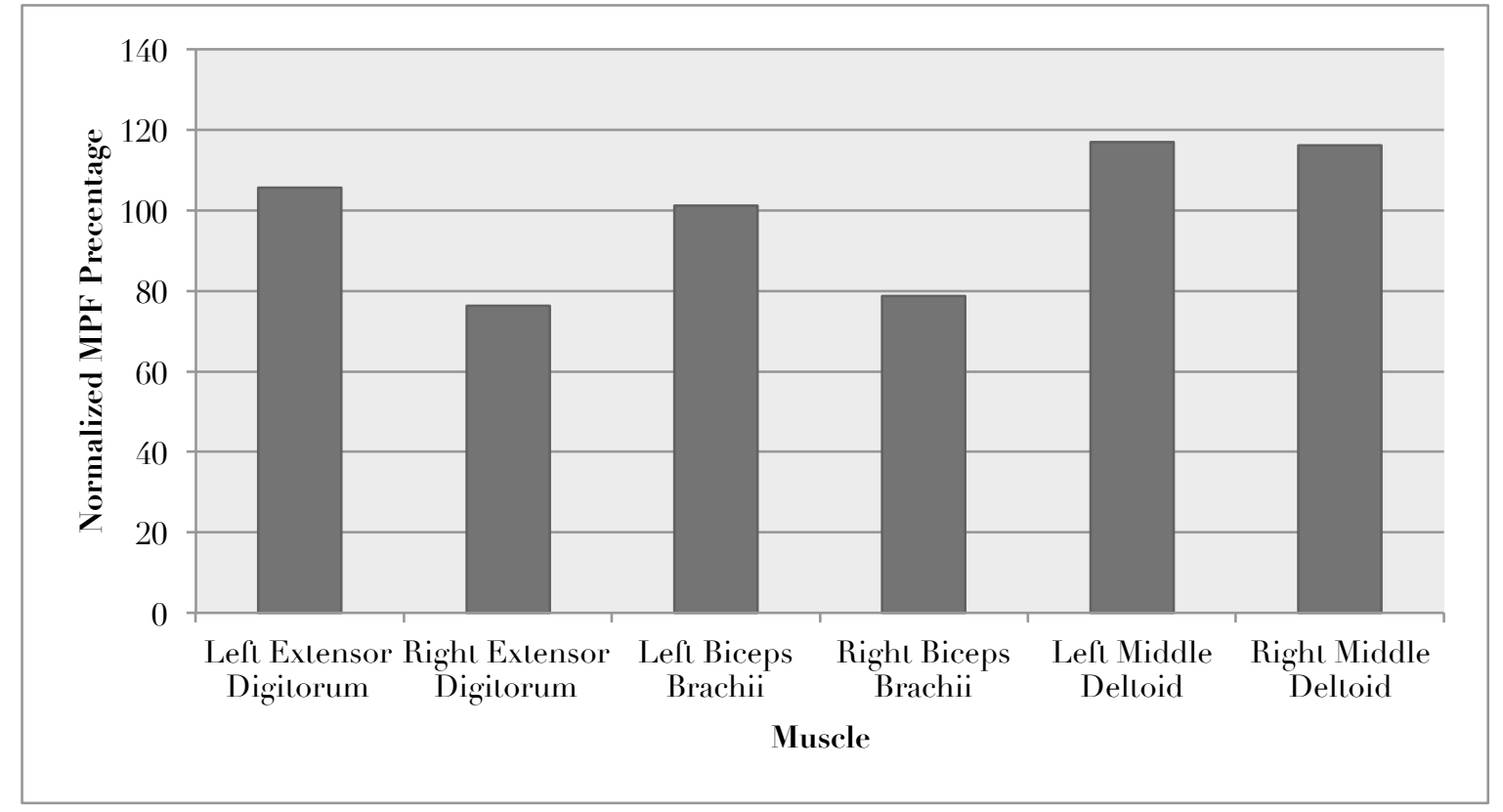

Figure 6-1: normalized MPF for the isometric contractions recorded for the six muscles after the task; shown as a percentage of the isometric contraction recorded before the task

As the figure illustrated, the lowest MPF percentage was noted for the right extensor digitorum at $76 \%$, followed by the right biceps brachii at $78 \%$. The middle deltoids were calculated with a $17 \%$ and $16 \%$ increase to the previously recorded isometric contraction. A slight decrease can be noticed for the left biceps brachii and extensor digitorum. A discussion of the results ensues in Section 6.5. 
The paired t-test was carried out between the isometric contractions recorded before and after the experimental task where a null hypothesis stated that the two means for the recorded contraction are equal. The p-values calculated did not show any evidence against the null hypothesis for any muscle, except for the left middle deltoid at 0.0635, which have shown slight evidence against the null hypothesis but not enough to reject it. Therefore, the difference between the captured electromyograms is statistically insignificant. The computed MPFs and the results of the paired t-test carried out for the six assessed muscles in the vertical condition are included in Appendix B.2.1.

\subsubsection{Electromyogram Results}

Data was also collected during the experimental task at 5 minutes intervals, where electromyograms were recorded for one minute. The data collected was passed through a low-pass filter and then the averaged RMS for each recording was calculated. The averaged RMS values were then normalized as a percentage of the isometric contraction recordings, which were collected prior to starting the task and processed in the same manner. Figure 6 2 shows the muscle activation for the right and left middle deltoids, grouped according to their muscle group, the time blocks are associated with their times in task and are found in Table 1 .

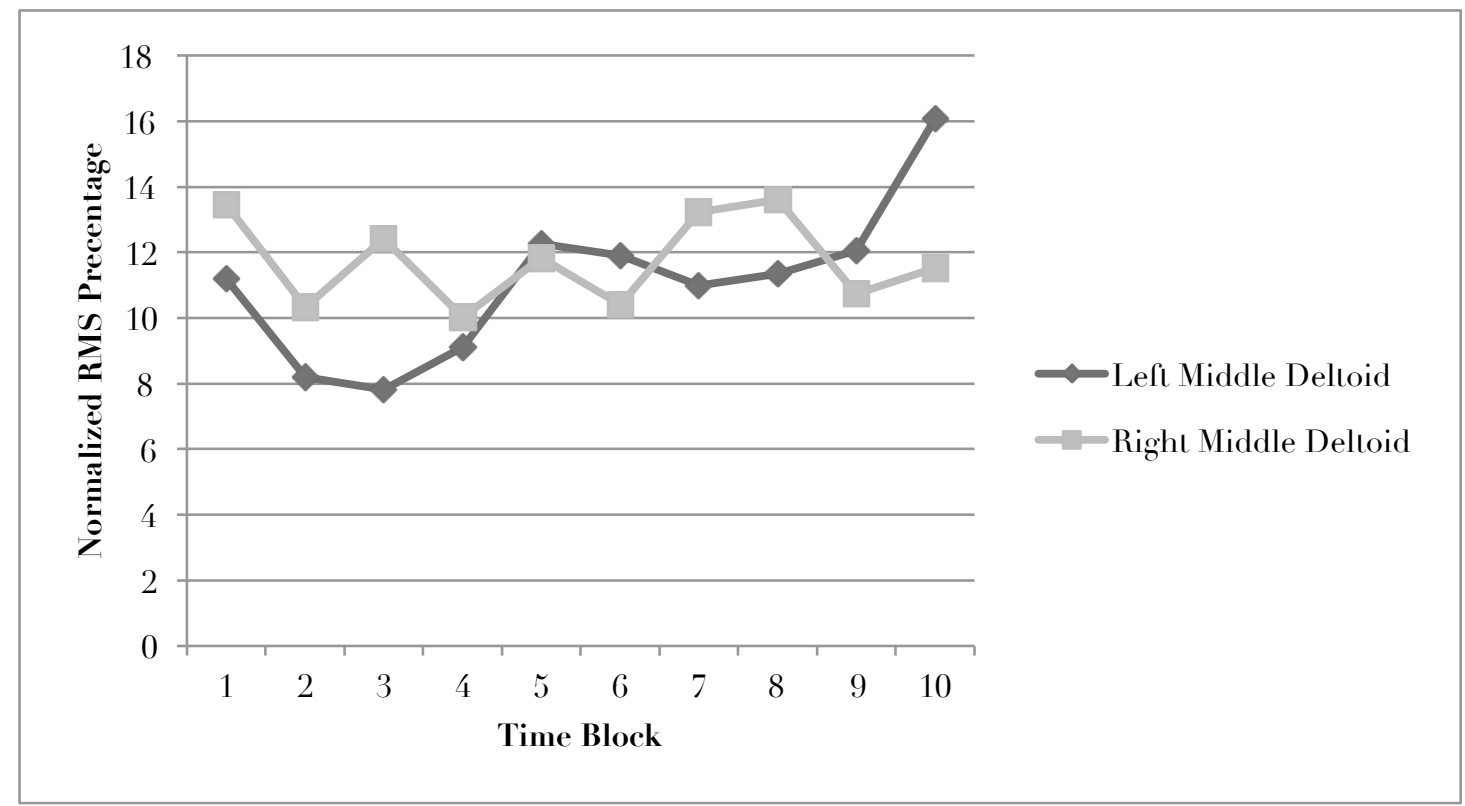

Figure 6-2: normalized RMS measured for the middle deltoids during the experimental task as a function of block

As it is shown in figure 6-2, the right and left middle deltoids are calculated to have similar levels of activations at $13 \%$ and $11 \%$ respectively. This is then followed by an equal decrease of $3 \%$ at the $10^{\text {th }}$ minute of the task. While the activation of the right middle deltoid increased, the left decreased by $1 \%$ but is then followed by an incline in the next two time blocks. At the $40^{\text {th }}$ and $46^{\text {th }}$ minute the activation stabilized at $13 \%$ for the right middle deltoid and $10 \%$ for the left. In the last recorded time block, higher muscle activation for the left middle deltoid is noted at $16 \%$, higher than its right counterpart at $11 \%$. 


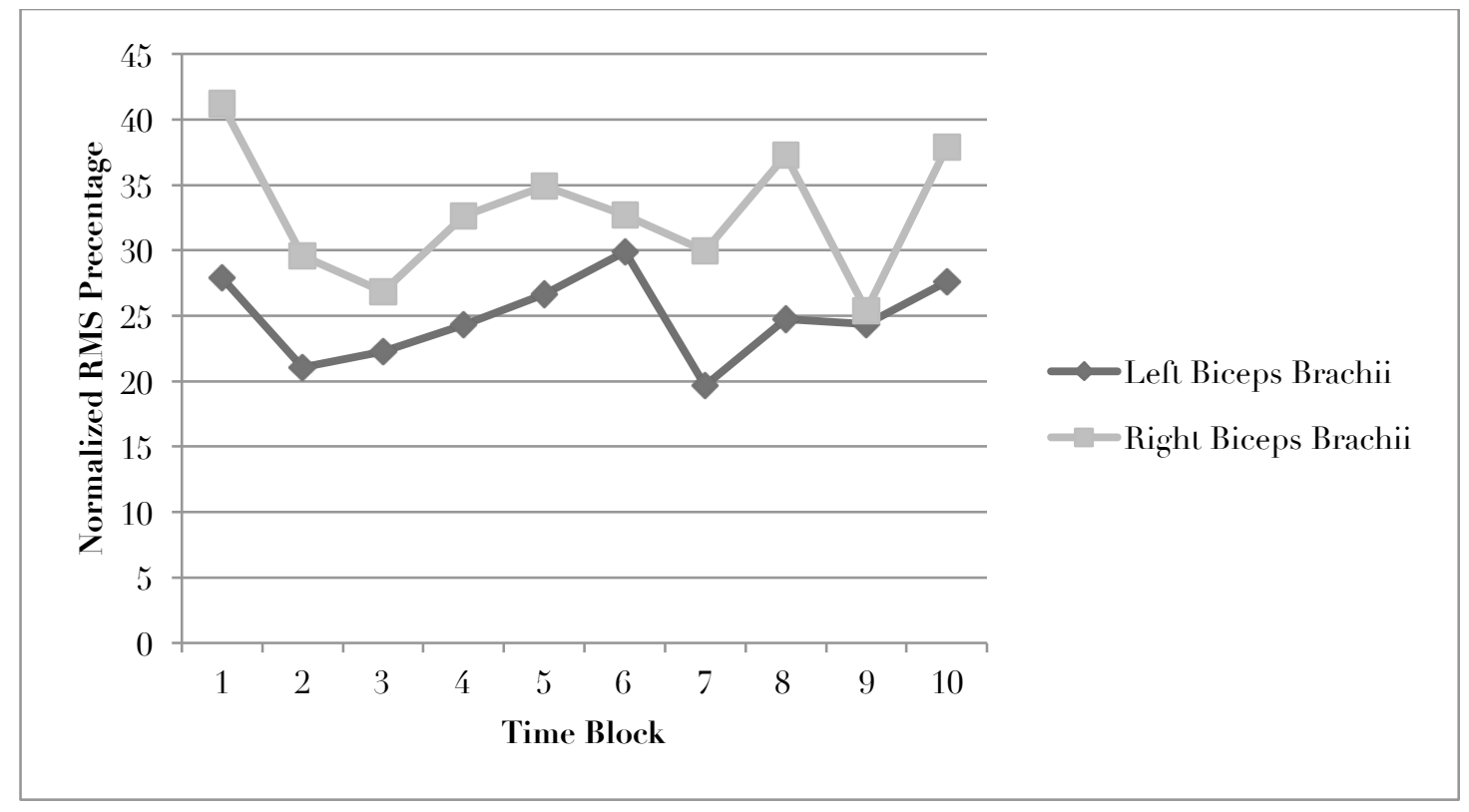

Figure 6-3: normalized RUS measured for the biceps brachii during the experimental task as a function of block

Figure 6-3 illustrates the RMS muscle activation percentages for the right and left biceps brachii starting at $41 \%$ and $27 \%$ respectively. After a slight decline at the $10^{\text {th }}$ minute, the left middle deltoid's activation level rose steadily until it reached $29 \%$ at the $34^{\text {th }}$ minute of the task; this is then followed by a sharp $10 \%$ decline in the next time block. A similar slope is noted for the right biceps brachii but at higher percentages, ranging from $25 \%$ and $37 \%$.

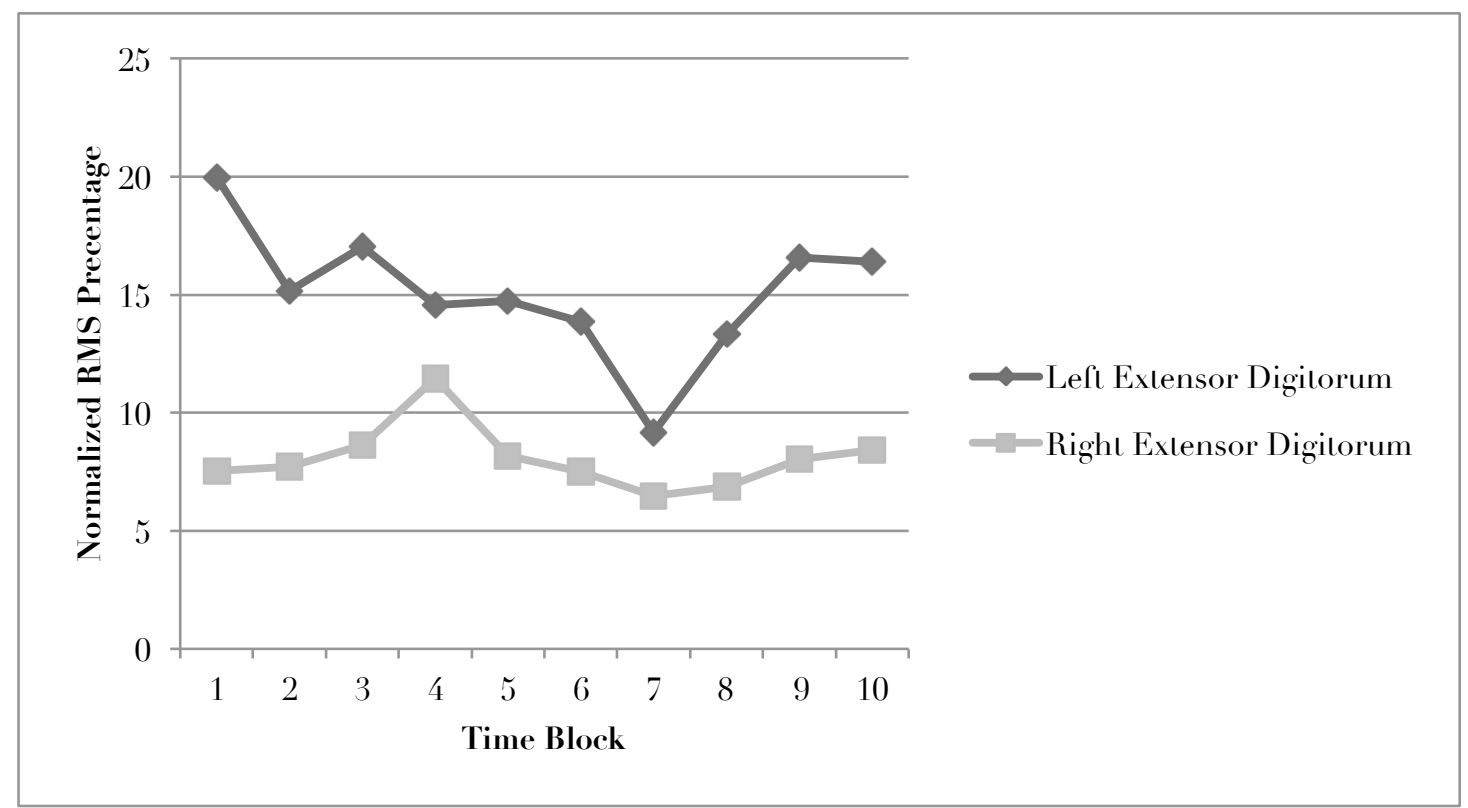

Figure 6-4: normalized RMS measured for the extensor digitorum during the experimental task as a function of block

The muscular activation of the right and left extensor digitorum is shown in Figure 6-4, with percentages starting at $7 \%$ and $19 \%$. Throughout the task the right middle deltoid activation levels are relatively stabilized with values ranging from $6 \%$ and $8 \%$, excluding the $22^{\text {nd }}$ minute of the task where the activation slightly rose to $11 \%$. Unlike its right counterpart, the left middle deltoid proved inconsistent with activation percentages ranging from $9 \%$ and $19 \%$. 
A one-way repeated ANOVA carried out between the ten-recorded measures in 5 minutes intervals have shown no evidence against the null hypothesis that states that the means of the ten recordings are equal for the extensor digitorum, biceps brachii, and the middle deltoid, thus proving statistically insignificant. The results of the ANOVA calculations carried out for the six assessed muscles in the vertical condition are included in Appendix B.3.1.

\subsubsection{Video Results}

The interactions with the experimental task on the tabletop were videotaped throughout for further analysis of preference of interaction techniques over time. At the beginning of the task, the posture adapted was that of a seating posture with the body slightly leaning forwards, however as time progressed changes were noted. Figure 6-5 shows the postures adopted by 4 of the 5 subjects with the palm of the left (non-dominant) hand resting on the side of the tabletop with the elbow locked straight or both elbows resting on the edge of the table closest to the subject without restricting the hands. Both postures were noted throughout the task, one instance of each posture was adapted early on with constant application, another instance showed posture adoption at the last third of the hour.
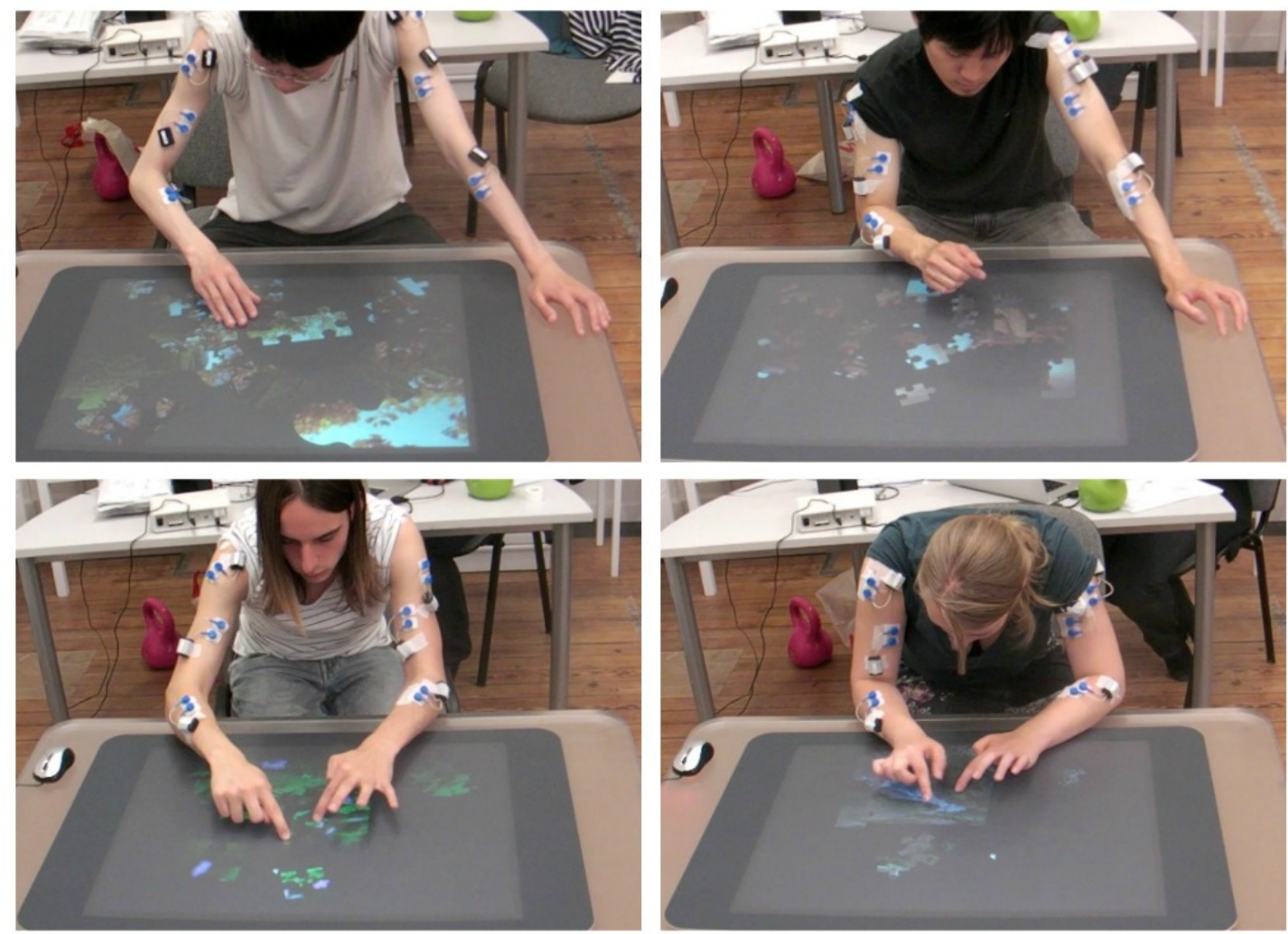

Figure 6-5: the resting postures adopted during the experimental task. The top panel shows the left (nondominant) arm resting on the side of the tabletop. The bottom panel shows both arms resting on top of the tabletop while interactions continues to ensue

A review of the videos has also shown that translations were carried out unimanually with multiple-fingers or one-finger mostly by the right (dominant) hand throughout the task. In two of the five cases, the right and left hand alternated the performance of translations; most importantly the hand carried out the piece translation closest to it. For some of the subjects, simultaneous unimanual multiple-fingers or one-finger translations were carried out by both hands, however, instances of occurrence were small. For scaling transformation a preference of symmetric bimanualism was noted for most subjects throughout the task with one-finger from each hand. In some cases, a puzzle piece was translated to a resting 
left hand to be scaled asymmetrically bimanually where the piece is locked into position with one-finger on one hand while the finger from the other hand applied the transformation. Nevertheless, instances of the previous case are fewer than that of removing the resting hand from the resting position to be used bimanually. No decrease of bimanualism is noted for scaling. The puzzle piece size was sometimes deterministic of bimanual or unimanual behavior on scaling transformations, where two subjects were noted to adopt bimanualism as the pieces got larger and unimanual interactions for smaller pieces.

Unimanual one-finger or multiple-finger rotation was frequently adopted throughout the task with occasional bimanual one-finger or multiple-finger from each hand rotation. The multiple-finger unimanual rotations were carried out with the two fingers acting symmetrically, which were also noted for bimanual one-finger or multiple-finger rotation where both fingers were moved while rotating the wrist to transform the puzzle piece. In one subject, bimanual one-finger rotations were adopted for the first tenth of the task but later dropped for its unimanual equivalent, unimanual multi-fingers rotation. The experimental task also allowed simultaneous rotation, scaling and translation (RST) transformations; most subjects using one-finger or multiple-fingers from each hand carried out RST bimanually in a symmetrical manner. Despite the adoption of multiple-finger transformations either unimanually or bimanually, most subjects resorted to one-finger interactions by the end of the task.

\subsection{Summary and Discussion}

As expected the subjective assessments, carried out before and after the task have proven the fatigability of the tabletop interaction over an hour-long period, however, no significant difference was noted statistically. The objective analysis of fatigue results has shown a decrease of the MPF for the electromyograms reordered before the task for the right extensor digitorum and biceps brachii. Thus the fatigability of those muscles is indicated due to a decrease in muscular discharge; however no decrease was noted for the right middle deltoid as was hypothesized. A paired t-test carried out afterwards has proven the difference to be statistically insignificant. The video analysis has shown the right side muscles were employed more extensively than that of the left side since unimanual translations and rotations were mostly performed by the right hand in addition to being used bimanually for scaling and RST. Nevertheless, bimanual scaling was not dropped for its unimanual counterpart as it was expected. In fact, the techniques adopted from the beginning of the task were maintained until the end.

The results of the recordings taken during the experimental task have correlated with the video analysis and the isometric contraction results. The latter showed a similar level of activation for both the left and right side muscles, indicating the maintainability of the technique obtained from the beginning until the end of the task, and more specifically bimanualism for scaling and RST. As it is shown in Figure 6-4, the percentage of muscle activation for the right extensor digitorum throughout the task is relatively stabilized which can be attributed, as the video results have shown, to a constant employment of the right extensor digitorum for the transformations used. This in turn explains the inconsistent activation of its left counterpart, where its employment is sporadic, changing from one interval to another depending on how far along the task; noticed behavior with the puzzle involved bimanually scaling the puzzle and then unimanually translating and rotating when looking for matching pieces and finally applying RTS transformation to connect the pieces. The muscle activation levels for middle deltoid and biceps brachii are shown in Figures 6-2 and 6-3 respectively, where a similar level of activation is maintained.

The sustainability of the techniques employed, as noted in the video results were also evident in the ANOVA test carried out between the different time blocks. Results of which 
have shown the statistical insignificance of the difference between the averaged RMS at the recorded intervals. It should be noted that the sensitivity of the electromyograph is not specific to the interactions with the tabletop, thus the measured values also include other none-interaction muscle activators. The recordings collected during the task and the video analysis have proven the sustainability of the adopted interaction techniques over the hourlong task. 


\section{Chapter 7}

\section{Experiment II}

\subsection{Introduction}

This chapter describes and analyzes the results of the experimental procedure performed for the vertical tabletop condition. The purpose of the experiment is defined in Section 7.2 followed by a list of hypothesized expectations. Section 7.4 describes the results, which are divided in subsections relevant to the dataset analyzed. Finally, the results are summarized and discussed in relation to the hypotheses stated in Section 7.3.

\subsection{Purpose of the Experiment}

The purpose of the experiment is to gain insight into the interaction techniques adopted during vertical tabletop interactions for an extended period of time. An ergonomics perspective is also captured where the muscles analyzed are objectively assessed for fatigue after the experimental task and muscle activation during that task. With such materials better and less harmful interactions can be designed for upcoming technology with the true potential of traditional workstation substitution.

\subsection{Hypotheses of Expected Results}

The following are the hypotheses of the expected results for the datasets obtained from the experimental session for the vertical tabletop condition identical to those listed in Section 6.1.2. The hypothesis for the subject ratings collected before and after the experimental task is that the averaged values collected after the task will be greater than that of the averaged before value, thus indicating the fatigability of the vertical tabletop interaction. Similar hypotheses are stated for the recorded isometric contraction of the six assessed muscles where the MPF calculated for the contractions recorded after the task are smaller than the ones captured and processed before the task, in turn proving the fatigability of that muscle. The hypotheses also state that fatigue is noted for the right (dominant side) extensor digitorum, middle deltoid, and biceps brachii, however none or less fatigue is noted for their left counterparts.

With respect to the recordings collected during the task at 5-minute intervals and the film analysis, it is hypothesized that as time progresses subjects will fall back on bimanual interaction techniques, as they are more convenient than unimanual techniques. The results of the video analysis should be evident in the recordings captured during the task, where a normalized activation is higher for the assessed right muscles than that of their left counterparts further along the task. 


\subsection{Results}

The following subsections review the results of the analysis of the four datasets described in Section 5.5: subjective ratings, isometric contraction electromyograms, electromyograms, and videos.

\subsubsection{Subjective Ratings Results}

The averaged values of the subjective ratings that were collected after the task proved higher than that of those collected before the task. A paired t-test was then carried out between the two datasets of overall perceived exertion recorded for the horizontal condition to prove statistical significance. The results have shown in regards to a null hypothesis that stated that the two means of the before and after sets are equal, that with $p=0.0127$, there is moderate evidence against the null hypothesis, and therefore it is rejected for the alternative hypothesis that states that the mean of the initial dataset is smaller than the later dataset. The detailed results are included in Appendix B.1.

\subsubsection{Isometric Contraction Electromyogram Results}

The isometric contraction electromyograms recorded before and after the experimental task were processed by means of rectification and filtering, the signal was then transformed from the time domain to the frequency domain where the MPF was calculated. The data captured after the task was then normalized as a percentage of the isometric contraction recorded prior to the task, where each value is representative of a MPF percentage for each muscle recorded. The results of the process briefly described above can be found in Figure $7-1$.

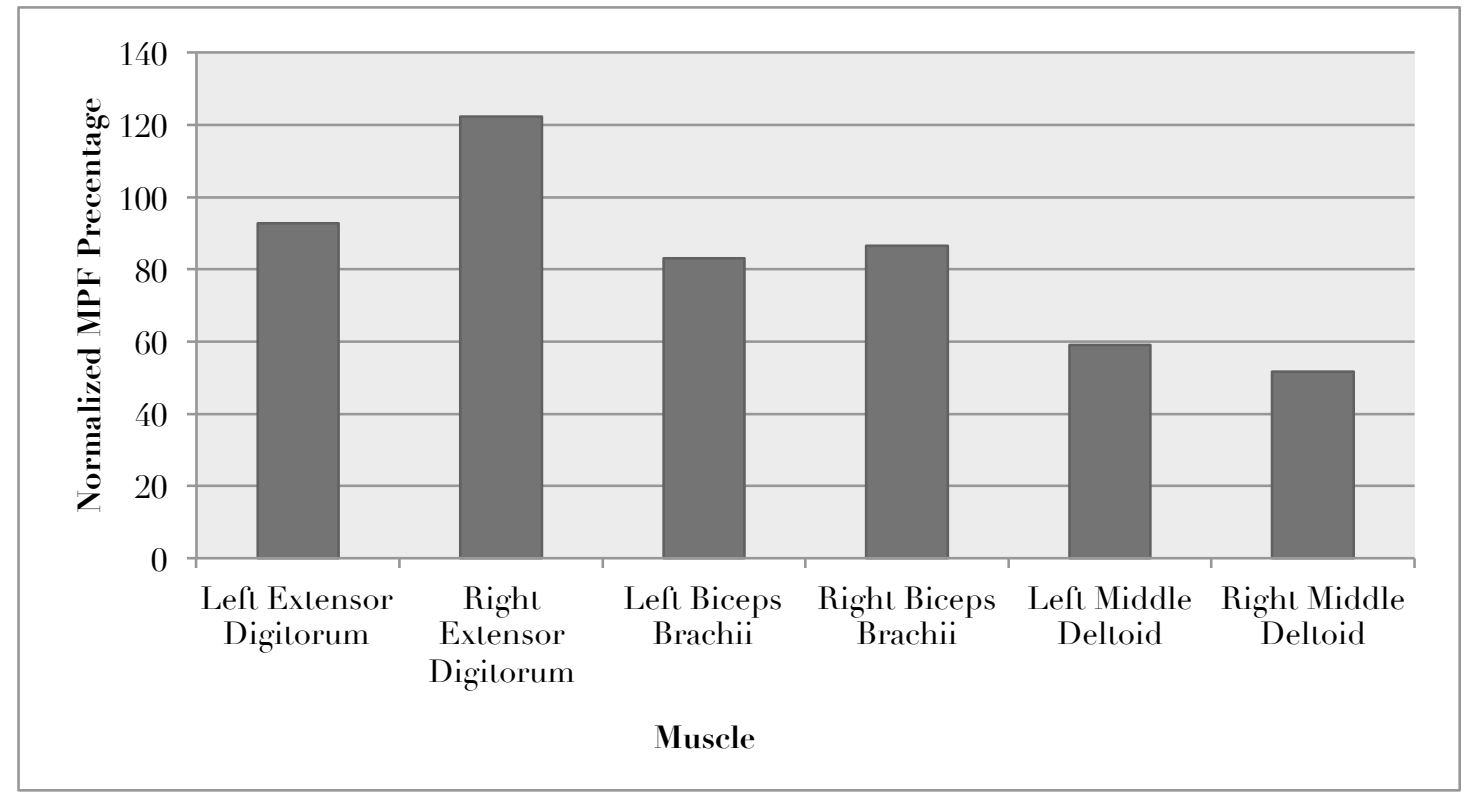

Figure 7-1: normalized MPF for the isometric contractions recorded for the six muscles after the task shown as a percentage of the isometric contraction recorded before the task

As a percentage of the isometric contraction recorded prior to the experimental task, the middle deltoids were recorded with the lowest MPF percentage at $51 \%$ and $58 \%$ for the right and left arms respectively. An increase is only noted for the right extensor digitorum with an increase of $22 \%$ that the original isometric contraction. Approximately similar 
percentages at $83 \%$ and $86 \%$ can be noticed for the left and right biceps brachii. An $8 \%$ decrease is also shown for the left extensor digitorum.

A paired t-test was then carried out between the isometric contractions recorded before and after the experimental task. The null hypothesis specified for the test stated that the two means for the recorded contractions are equal. The calculated p-values for all measured muscles did not show evidence against the null hypothesis, thus proving the changes statistically insignificant. The computed MPF and the results of the paired t-test carried out for the six assessed muscles in the vertical condition are included in Appendix B.2.2.

\subsubsection{Electromyogram Results}

The data collected during the experimental task was passed through a low-pass filter and from the time domain the averaged RMS calculated. The values were then normalized as a percentage of the isometric contraction recordings collected prior to the experimental task. The isometric contraction electromyograms were also processed in the same manner. The muscle activation of the six measured muscles, at 5 minute intervals during the task is shown in Figures 7-2 to 7-4 where they are grouped according to muscle type. Table 1 in Section 6.4.3 displays the numbered intervals and the times frames they represent.

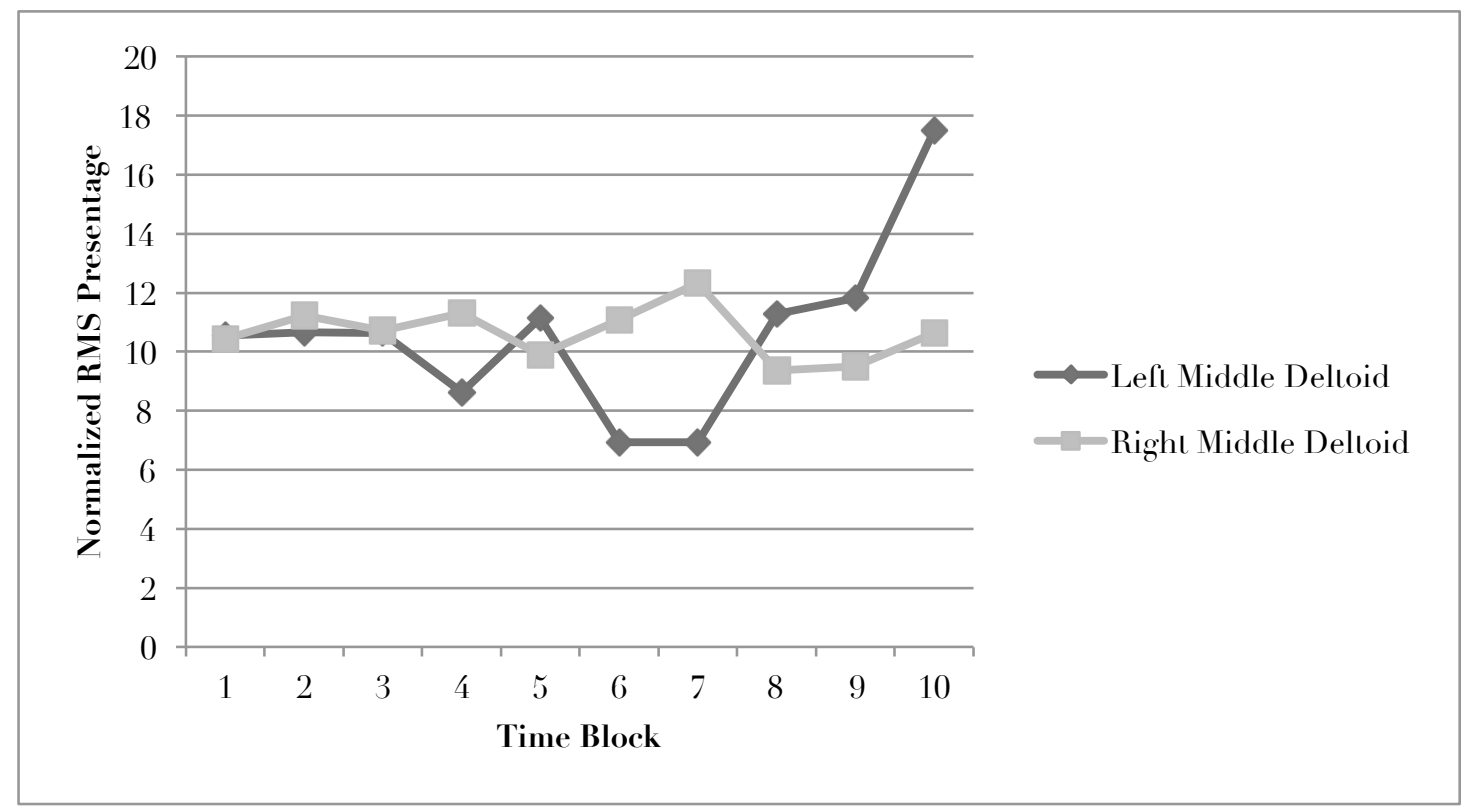

Figure 7-2: normalized RMS measured for the middle deltoids during the experimental task as a function of block

Figure 7-2 shows the activation level of the right and left middle deltoids calculated as a percentage of isometric averaged RMS. A similar level of activation is noted for the right and left middle deltoid for the first half of the task with values ranging from $8 \%$ to $11 \%$. At the $34^{\text {th }}$ minute of the task a $5 \%$ decline is noted for the left middle deltoid where it maintained for the next time blocks followed by a relatively steady incline until the end of the task where it reached $17 \%$ activation. Unlike its left counterpart, the right middle deltoid maintained a relatively stable activation with values ranging from $9 \%$ and $12 \%$. 


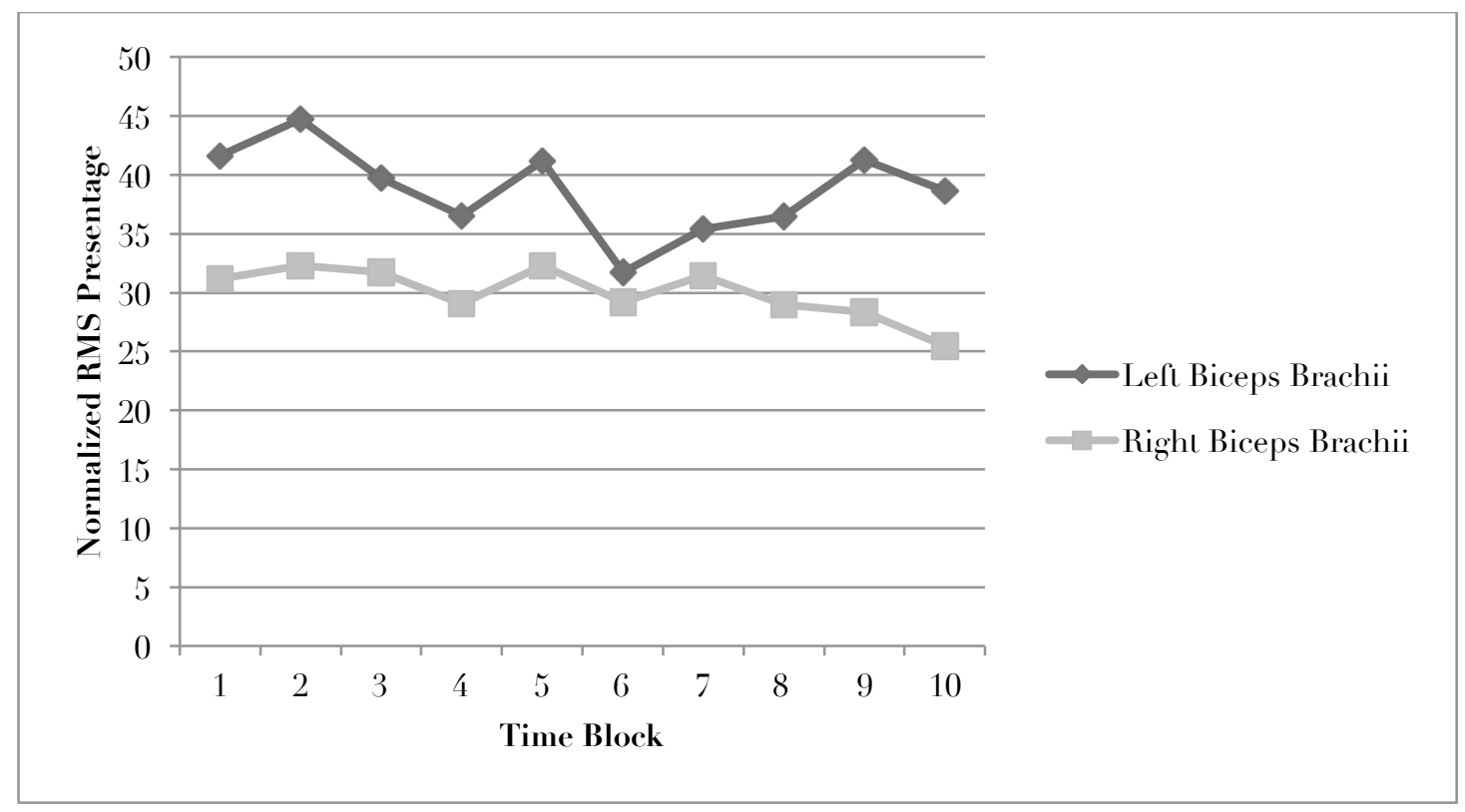

Figure 7-3: normalized RMS measured for the biceps brachii during the experimental task as a function of block

Figure $7-3$ shows the activation level of the right and left biceps brachii muscles starting at $41 \%$ and $31 \%$ respectively. The right biceps brachii is relatively stable throughout the task with $30 \%$ activation give or take $2 \%$. Its left counterpart, however, is shown to be inconsistent with a $3 \%$ decline between the $10^{\text {th }}$ and $22^{\text {nd }}$ minute of the task, which then slightly increased but is then followed by a further $2 \%$ decline in activity at the $40^{\text {th }}$ minute. It later maintained a relatively steady incline until a slight drop is noted at the last recorded time block.

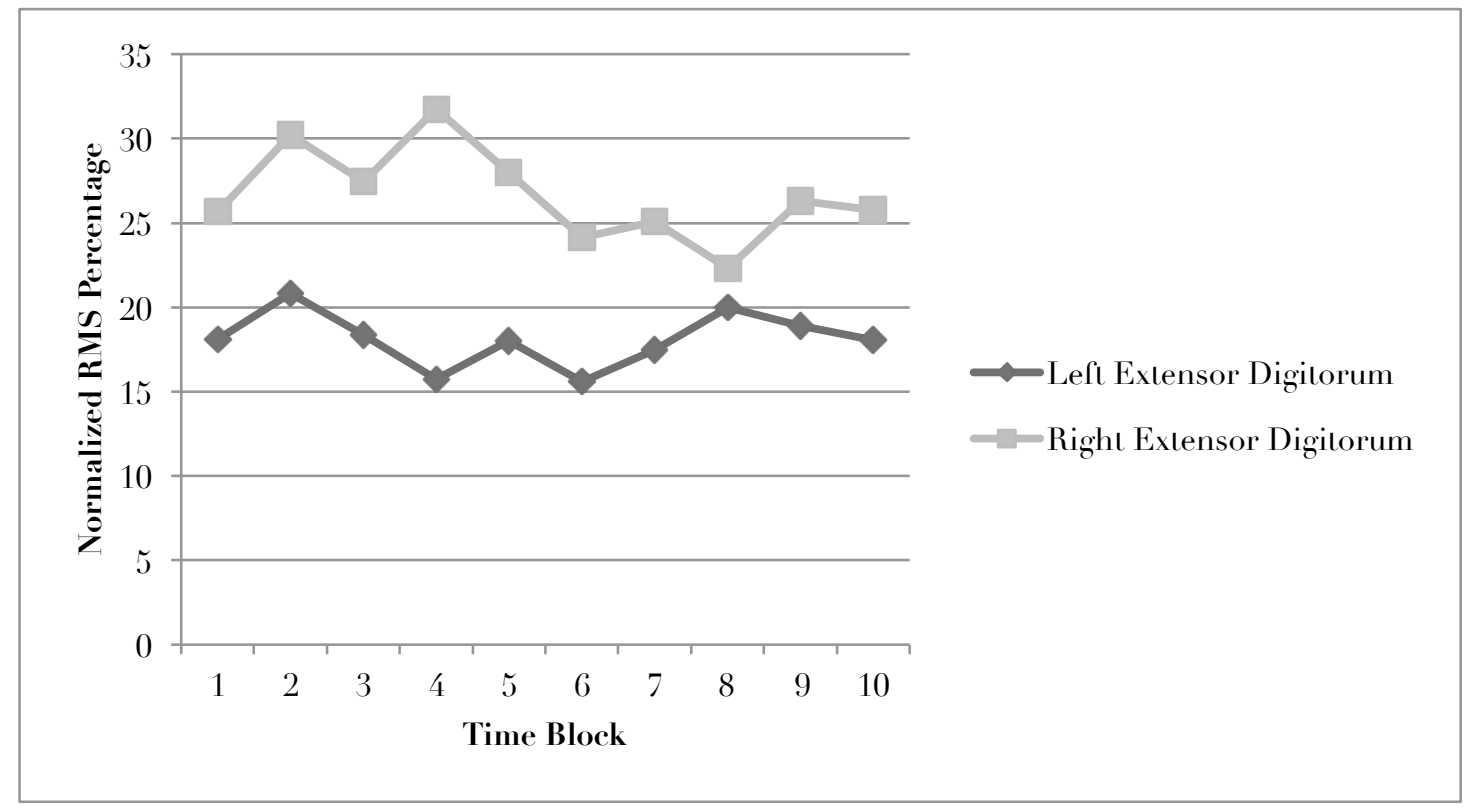

Figure 7-4: normalized RMS measured for the extensor digitorum during the experimental task as a function of block

As it is shown in Figure 7-4, a 10\% gap is noted between the right and left extensor digitorum where the right muscle maintains higher activation rates than its left counterpart. The activation levels of the left extensor digitorum ranges between $15 \%$ and $20 \%$ activation throughout the task where the highest decline is noticed between $10^{\text {th }}$ and $22^{\text {nd }}$ minute of the task and similarly a higher incline between the $34^{\text {th }}$ and $46^{\text {th }}$ minute is noted. The right extensor digitorum activation values are maintained between the $25 \%$ and $30 \%$ in the first 
half of the task. However the range then declined at the second half with activation levels ranging from $22 \%$ and $26 \%$, with a last recorded activation at the $58^{\text {th }}$ minute of $25 \%$.

A one-way repeated ANOVA was then carried out between the ten-recorded measures in 5 minutes intervals. No evidence was shown against the null hypothesis that stated that the means of the ten recording are equal for the biceps brachii, right middle deltoid, and both extensor digitorum. However, a statistically significant difference was noted for the left middle deltoid thus the null hypothesis is rejected for the alternative for that muscle. Looking at the means it is noted that the interactions carried out on the sixth recording in the $34^{\text {th }}$ minute $\left(6^{\text {th }}\right.$ block) of the experimental task is the lowest of all activation, thus attributing to the rejection of the hypothesis. The results of the ANOVA calculations carried out for the six assessed muscles in the vertical condition are included in Appendix B.3.2.

\subsubsection{Video Results}

For the vertical tabletop sessions, the subjects were videotaped during their interactions with the experimental task on a vertical tabletop. A review of those videos has shown a unimanual one-finger or multiple-finger adoption for translation by both hands, where the hands alternated translation transformations while the unused hand rested on the subject's lap. A posture commonly adopted by half of the subjects was that of resting the left foot on the right knee providing an elevated resting area for the left hand. This posture is shown in Figure 7-5. For scaling transformations, bimanual one-finger conditions were prominently used by most subjects and maintained throughout the experimental task. Instance of bimanual multiple-finger from each hand scaling was noted for two of the subjects but was later dropped for bimanual one-finger interactions. Occasional use of unimanual multiplefingers scaling was also noted but not as frequent as its bimanual counterpart. As a true representation of the scaling gesture it was carried out symmetrically. The size of the puzzle piece did not hold significance to the type of interaction adopted. For one subject, scaling transformations were carried out bimanually at the beginning of each puzzle where all puzzle pieces were scaled bimanually with one-finger form each hand, and unimanually with either the left or right hand when matching the pieces together.
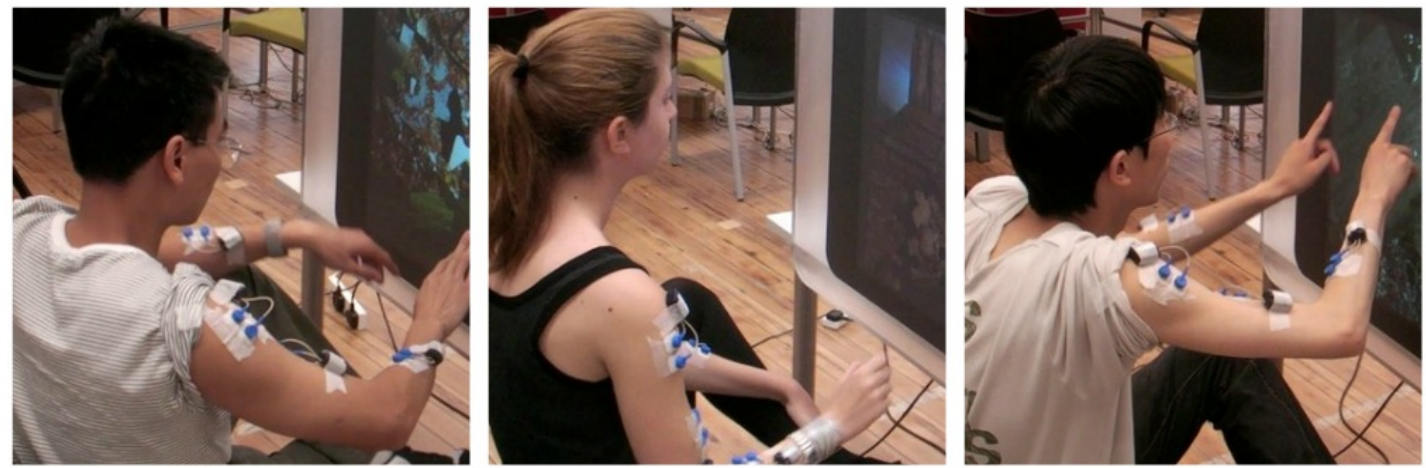

Figure 7-5: the posture adopted by 3 of the 6 subjects participating in the experiment, where the crossed leg is used as a resting place for the left (non-dominant) arm

Of the six subjects, half adopted bimanual one-finger or multiple-finger rotations and maintained that behavior with small instances of unimanual rotations throughout the task. The other half of the participants, adopted unimanual one-finger or multiple-finger rotations for their interactions with the vertical tabletop and maintained that performance until the end of the task. The unimanual section of the group has also shown occasional adoption of bimanualism for scaling but the adoption was not maintained at the last third of the allotted experimental time. The multiple-finger unimanual rotations were carried out with the two fingers acting symmetrically, which were also noted for bimanual one-finger or 
multiple-finger rotation where both fingers are moved while rotating the wrist to transform the puzzle piece.

Similar to the behavior adopted for scaling transformations, RST was performed bimanually using one-finger or multiple-fingers from each hand. Multiple-finger unimanual RTS adoption was only noted for two of the subject at the first half of the experimental task, after which bimanualism proved highly adoptive for RTS transformation throughout the task. For all subjects little or no behavioral change was noticed between the first and last half of the task, however, as was mentioned above postural changes were noted for three subjects as is shown in Figure 7-5.

\subsection{Summary and Discussion}

It was expected that the subjective ratings collected before and after the experimental task would differ and the before average value would prove higher than the before value. The calculated average of those two recordings have met expectations. The difference was statistically proven significant with a paired t-test that has shown moderate evidence against the equality of those values sufficient to adopt the alternative hypothesis that states an increased average for the electromyograms recorded after the task. Similar to the subjective ratings, isometric contractions were recorded before and after the task to assess the fatigability of the vertical tabletop interactions. The results have shown decreases in the normalized MPF percentages for both middle deltoids, both biceps brachii, and the left extensor digitorum, whereas a slight increase in the calculated percentage for the right extensor digitorum was seen. In Section 7.3, a decrease was expected for the assessed muscles located on the right side with little or no decrease for their left counterparts; however, the results only hold that expectation for the right extensor digitorum, not the right biceps brachii and extensor digitorum. The lowest MPF values were calculated for both middle deltoids and both biceps brachii, which signifies the fatigability of the vertical tabletop interaction over time for those two muscles. A paired t-test carried out to provide for statistical significance of the difference between the average did not give any evidence against the null hypothesis that stated their equality.

The video analysis has shown the adoption of bimanual techniques for scaling, RST and in some subjects for rotation as well, however, no decline in their behavior was noticed as it was expected. For rotation transformation those who did not adopted bimanualism but unimanual techniques, occasionally incorporated bimanualism but later dropped it for its unimanual counterpart. The more extensive adoption of unimanual technique, as opposed to the horizontal condition, can be noted in the activation levels shown in Figure 7-4 for the extensor digitorum where the activation level of right side is relatively higher than the left side throughout task. However, this is note noted for the biceps brachii where the right side is activated at a relatively stable level whereas its left counterpart is considerably inconsistent. This in turn can account for the stable adoption of the right hand throughout the task. The one-way repeated ANOVA, calculated for the right middle deltoid, extensor digitorum and the biceps brachii, proved statistically insignificant thus indicating the sustainability of the activation levels throughout the experimental task for those muscles. A statistical significance, however, was noted for the left middle deltoid, which signifies that the activation levels for the muscle were not maintained throughout the task. Thus meeting this thesis's expectation of a decline in bimanualism as the task progresses. It should be noted here, as it was in Section 6.5, that the sensitivity of electromyograph is to all forms of activities, including tabletop interactions, but not limited to it. 


\section{Chapter 8}

\section{Conclusion}

\subsection{Introduction}

This chapter provides a summary of the research and experimentation conducted by answering the three research questions in the following subsections that was presented in Section 1.4. Critical remarks on the conducted experiments are also put forth in Section 8.2. The chapter concludes by summarizing the answered research questions and considers future research relevant to the thesis goal.

\subsection{Answering the Research Questions}

\subsubsection{Question I}

The first research question posed in Chapter 1 asks: what interaction techniques are adopted for tabletop interactions when unimanual and bimanual techniques are made available?

An experimental task was developed utilizing bimanual and unimanual interaction techniques for scaling, translation, and rotation. During the experiments, the subjects were informed of such techniques and were only asked to complete the task any way possible. The video analysis carried out for the horizontal condition, showed a default adoption of bimanualism for scaling and RST, whereas unimanual techniques were used for translation and rotation. The scaling technique adopted was inherently symmetrical, however, it was noted for some incidents that asymmetrical application had been adopted as noted in Section 6.4.4. The other techniques were adopted as they were intended to be used. The analysis of the videos recorded for the vertical session have shown similar results but with bimanualism adopted by half of the six subjects for rotation as well. These results prove the naturalness of bimanualism for application as is noted in bimanual literature, but still presents unimanual techniques as indispensable.

\subsubsection{Question II}

The second research question asks: are the adopted techniques, be it bimanual or unimanual, sustainable throughout the hour-long task?

In the experiments, the sustainability of the techniques is assessed by viewing video recording of the experimental session and analyzing objective electromyograms of muscle activity during the task. The video analysis have proven the sustainability of the techniques adopted for the duration of the task for the horizontal condition, however, resting postures were adopted during the task in one case at the $10^{\text {th }}$ minutes of the hour-long task. The recorded muscle activation during the task prove the employment of bimanualism throughout the experimental task where activation measures are viewed for both the right and left sides, however, the recording can be misread as the electromyograph was sensitive to any form of activity not only to the interaction techniques. Nevertheless, a one-way 
repeated ANOVA was carried out to determine the equality or inequality of the averaged muscle activation levels calculated as mean RMS. The results of the statistical analysis found no significant difference between the averaged values collected over time, thus further providing insight into the sustainability of the techniques over time.

For the vertical sessions, the sustainability of the techniques was noted in the video analysis for scaling, translation, and RST. However, in the case of unimanual rotations adopted by three of the six subjects, an attempt at bimanualism was made for the first half of the task, where it was later dropped for its unimanual counterpart. A one-way ANOVA was also calculated, where the results have shown no evidence against the equality of the means of the different intervals for five of the six muscles, whereas the left middle deltoid showed a statistically significant difference between the means.

\subsubsection{Question III}

The third research question asks: are tabletop interaction techniques fatiguing over an hour-long task?

To assess fatigue, subjective and objective measures were used and the collected data was later analyzed for assessment in both conditions. Subjective ratings were collected before and after the experimental task where the means were calculated for both sets followed by a paired t-test to assess the difference's statistical significance. For the horizontal, despite there being a difference between the means of the before and after ratings (before < after) no statistical significance of that difference was noted. However, a statistical significance was identified for the vertical condition given the subjective ratings. Those results indicate the fatigability of the vertical condition but not the horizontal condition. Following a similar technique, the difference between the isometric recordings collected before and after the task proved statistically insignificant for both conditions.

\subsection{Critical Remarks on the Experiments}

A major concern lies within the recordings captured during the task, where 1-minute recordings were collected every 5 minutes. The purpose of the measurement was to objectively quantify muscle activation of the interaction techniques thus enabling the assessment of the sustainability of the different interaction techniques over time based on the activated muscles. However, the subjects were advised to interact with the tabletop as naturally as possible in order to not restrict behavior, this unfortunately introduced activations that are captured but not relevant to the measured interaction techniques, for example a subject brushing her hair behind her ear activates the extensor digitorum and middle deltoid muscles of that arm.

Technical difficulties relating to the hardware used may have interrupted the flow of interaction carried out by the subject. Two instance from each condition were noticed where subject have had difficulties with moving the puzzle pieces, i.e. touch sensing was lagging, and translation manipulations were employed more frequently in hopes of moving that piece. Another difficulty introduced by the software developed for the experimental task was when the pieces, despite being of the same orientation and size, failed to connect unless further manipulation was carried out. When faced with such difficulty the subjects were advised to attempt attaching the pieces together at a different scale.

The paired t-test and one-way repeated ANOVA results carried out for the subjective and objective data collected before and after the experimental task and also the recordings captured during the task have mostly proven insignificant. This can be due to the small sample size assessed, 5 and 6 subjects for the horizontal and vertical condition respectively. Therefore a power analysis using G*Power was conducted on both subjective and objective 
statistically analyzed data; the results of the power analysis on the collected subjective measure have calculated 6 and 12 subjects for the vertical and horizontal conditions respectively. A similar analysis carried out for the isometric contractions have shown that a sample size of 42 is needed to statistically signify the difference between the two means for the extensor digitorum and the middle deltoid. For the biceps brachii, a sample size as large as 400 is required to note any significant results.

A final concern to address is the evaluation methodology applied for the analysis of the video recording. The purpose of the analysis was to determine the techniques employed throughout the task. The methodology used in this thesis was to view the recordings while writing down general notes for every 5 -minute interval. The results of the methodology are included in Appendix B. Despite the effectiveness of the technique, the task proved tedious and time consuming, thus a better approach would have been suitable. For example, sampling the video at 5-minute interval might have produced similar results but have taken less time.

\subsection{Summary of Conclusion}

The general conclusions that can be drawn from the research and experimentation applied in this thesis are listed as follows:

1. The natural adoption of bimanualism using one-finger or multiple-finger from each hand for scaling and RST throughout an hour-long task

2. The natural adoption of unimanual one-finger interaction techniques for translation and rotation throughout an hour-long task

3. The sustainability of the interaction techniques adopted at the beginning of the task until the end of the hour-long task

4. The first experiment has shown evidence of physical workload increase after the experimental task, but it was proven statistically insignificant

5. The second experiment has shown evidence of physical workload increase after the experiments, which has proven to be statistically significant

6. The first experiment has shown evidence of fatigue for the right extensor digitorum and biceps brachii

7. The second experiment have shown evidence of fatigue for the middle deltoids, biceps brachii, and the left extensor digitorum

8. In the first experiment, the ANOVA test showed no significant evidence against the equality between the means of the different intervals for all six muscles. Thus proving the sustainability of the muscle activity over the one-hour long task

9. In the second experiment, the ANOVA test showed no significant evidence against the equality between the means of the different intervals for five of the six muscles. Statistically significant evidence is found for the left middle deltoid. Thus proving the sustainability of the muscle activity over the one-hour long task for five of the muscles

The aims and objectives of this project stated in Chapter 1 have essentially been met, and in the process have proven the applicability of electromyography for the objective assessment of fatigue for tabletop interaction techniques. The critical assessment carried out in Section 8.2 provides a number of ideas for future work. Most importantly, carrying out the analysis with a sample size that can provide for statistically significant difference between the means calculated for the before and after isometric contraction recordings. In the experiments, the same task was employed in both conditions, however the appropriateness of the task for vertical and horizontal application can be questioned. Thus future research that addresses the separate needs of the two condition and design a task appropriately can prove beneficial. Another interesting, yet tremendous, extension to this thesis would be to design and develop electromyograph transmitters that can be worn as 
accessories. This allows the recording of muscle activity in natural settings for longer periods time without hampering the task at hand. 


\section{Bibliography}

[1] V. Bush, “As we may think,” The Atlantic Monthly, vol. 176, no. 1, pp. 101-108, Jul-1945.

[2] P. Wellner, DigitalDesk, no. 330. Cambridge, United Kingdom: , 1994, p. 107.

[3] P. Dietz and D. Leigh, "DiamondTouch: a multi-user touch technology," in Proc. 14th Annu. ACM Symp. on User Interface Software and Technology, 2001, pp. 219-226.

[4] D. Wigdor, G. Penn, K. Ryall, A. Esenther, and C. Shen, "Living with a Tabletop: Analysis and Observations of Long Term Office Use of a Multi-Touch Table," in Proc. 2nd Annu. IEEE Int. Workshop on Horizontal Interactive Human-Computer Systems, 2007, pp. 60-67.

[5] A. Mazalek, M. Reynolds, and G. Davenport, "The TViews Table in the Home," in Proc. 2nd Annu. IEEE Int. Workshop on Horizontal Interactive Human-Computer Systems, 2007, pp. 5259.

[6] M. R. Morris, A. J. B. Brush, and B. R. Meyers, "A field study of knowledge workers\&\#39; use of interactive horizontal displays," in Proc.3rd Annu. IEEE Int. Workshop on Horizontal Interactive Human-Computer Systems, 2008, pp. 105-112.

[7] D. Saffer, Designing Gestural Interfaces: Touchscreens and Interactive Devices. O'Reilly Media, Inc., 2008.

[8] R. J. K. Jacob, "New human-computer interaction techniques," in Human-Machine Communication for Educational Systems design, ed. M.D. Brouver-Janse and T. K. Harrington, Springer-Verlag, 1994, pp.131-138

[9] B. Shneiderman, Designing the User Interface, vol. 3, no. 3. Addison-Wesley Reading, MA, 1987, p. 145.

[10] W. Buxton, "A Touching Story : A Personal Perspective on the History of Touch Interfaces Past and Future Lost Along the Way," Society for Information Display Symposium Digest of Technical Papers, vol. 41, no. 1, pp. 444-448, 2010.

[11] L. Kasday, “Touch position sensitive surface,” U.S. Patent 44841791984.

[12] S. K. Lee, W. Buxton, and K. C. Smith, "A multi-touch three dimensional touch-sensitive tablet," in Proc. SIGCHI Conf. on Human Factors in Computing Systems, 1985, no. April, pp. 21-25.

[13] W. Buxton, "Combined keyboard/touch tablet input device," XEROX Disclosure Journal, vol. 19, no. 2, pp. 109-111, 1994.

[14] W. Buxton, "Multi-touch systems that I have known and loved," 2007. [Online]. Available: http://www.billbuxton.com/multitouchOverview.html. [Accessed: 14-Jun-2011].

[15] W. Buxton, "The Active Desk," 2009. [Online]. Available: http://www.billbuxton.com/ActiveDesk.html. [Accessed: 28-Jun-2011]. 
[16] A. D. Wilson, "PlayAnywhere: a compact interactive tabletop projection-vision system," in Proc. 18th Annu. ACM Symp. on User Interface Software and Technology, 2005, pp. 83-92.

[17] A. Sears and B. Shneiderman, "High precision touchscreens: design strategies and comparisons with a mouse," International Journal of Man-Machine Studies, vol. 34, no. 4, pp. 593-613, 1991 .

[18] C. Forlines, D. Wigdor, C. Shen, and R. Balakrishnan, "Direct-touch vs. mouse input for tabletop displays," in Proc. SIGCHI Conf. on Human Factors in Computing Systems, 2007, pp. 647-656.

[19] S. Meyer, O. Cohen, and E. Nilsen, "Device comparisons for goal-directed drawing tasks," in Proc. Conf. Companion on Human Factors in Computing Systems, 1994, pp. 251-252.

[20] T. Moscovich and J. F. Hughes, "Indirect mappings of multi-touch input using one and two hands," in Proc. SIGCHI Conf. on Human Factors in Computing Systems, 2008, pp. 12751283 .

[21] W. Buxton and B. Myers, "A study in two-handed input," in Proc. SIGCHI conf. on Human Factors in Computing Systems, 1986, vol. 17, no. 4, pp. 321-326.

[22] Y. Guiard, "Asymmetric division of labor in human skilled bimanual sction: the kinematic chain as a model," Journal of Motor Behavior, vol. 19, pp. 486-517, 1987.

[23] E. A. Bier, M. C. Stone, K. Pier, W. Buxton, and T. D. DeRose, "Toolglass and magic lenses: the see-through interface," in Proc. 20th Annu. Conf. on Computer Graphics and Interactive Techniques, 1993, no. Mmm, pp. 73-80.

[24] P. Kabbash, W. Buxton, and A. Sellen, "Two-handed input in a compound task," in Proc. SIGCHI Conf. on Human Factors in Computing Systems, 1994, pp. 417-423.

[25] S. Zhai, B. A. Smith, and T. Selker, "Improving browsing performance: s study of four input devices for scrolling and pointing tasks," in Proc. IFIP TC13 Int.Conf. on Human-Computer Interaction, 1997, vol. 17, pp. 286-293.

[26] R. Balakrishnan, G. W. Fitzmaurice, G. Kurtenbach, and W. Buxton, "Digital tape drawing," in Proc. 12th Annu. ACM Symp. on User Interface Software and Technology, 1999, vol. 1, pp. 161-169.

[27] T. Grossman, R. Balakrishnan, G. Kurtenbach, G. W. Fitzmaurice, A. Khan, and W. Buxton, "Interaction techniques for 3D modeling on large displays," in Proc. 2001 Symp. on Interactive 3 D Graphics, pp. 17-23.

[28] T. Grossman, R. Balakrishnan, G. Kurtenbach, G. W. Fitzmaurice, A. Khan, and W. Buxton, "Creating principal 3D curves with digital tape drawing," in Proc. SIGCHI Conf. on Human Factors in Computing Systems, 2002, no. 4, pp. 121-128.

[29] C. D. Shaw and M. Green, "THRED: a two-handed design system," Multimedia Systems, vol. 5, no. 2, pp. 126-139, Mar. 1997.

[30] X. Jiao, H. Deng, and F. Wang, “An investigation of two-handed manipulation and related techniques in multi-touch interaction," in Proc. Int. Conf. on Machine Vision and Humanmachine Interface, 2010, pp. 565-568.

[31] A. Leganchuk, S. Zhai, and W. Buxton, "Manual and cognitive benefits of two-handed input: An experimental study," ACM Transactions on Computer-Human Interaction, vol. 5, no. 4, pp. 326-359, Dec. 1998. 
[32] D. Casalta, Y. Guiard, and M. Beaudouin-Lafon, "Evaluating two-handed input techniques: Rectangle editing and navigation," in Proc. SIGCHI Conf. on Human Factors in Computing Systems (Extended Abstracts), 1999, no. May, pp. 236-237.

[33] R. Owen, G. Kurtenbach, G. W. Fitzmaurice, T. Baudel, and W. Buxton, "When it gets more difficult, use both hands: exploring bimanual curve manipulation," in Proc. Graphics interface, 2005, pp. 17-24.

[34] K. Hinckley, M. Czerwinski, and M. Sinclair, "Interaction and modeling techniques for desktop two-handed input," in Proc. 11th Annu. ACM Symp. on User Interface Software and Technology, 1998, pp. 49-58.

[35] S. Zhai, E. Kandogan, B. a Smith, and T. Selker, "In search of the 'magic carpet': design and experimentation of a bimanual 3D navigation interface," Journal of Visual Languages \& Computing, vol. 10, no. 1, pp. 3-17, Feb. 1999.

[36] C. Latulipe, S. Mann, C. S. Kaplan, and C. L. A. Clarke, "symSpline: symmetric two-handed spline manipulation," in Proc. SIGCHI Conf. on Human Factors in Computing Systems, 2006, pp. 349-358.

[37] K. Hinckley, R. Pausch, J. C. Goble, and N. Kassell, "Passive real-world interface props for neurosurgical visualization," in Proc. SIGCHI Conf. on Human Factors in Computing Systems, 1994, pp. 452-458.

[38] K. Hinckley, R. Pausch, and D. Proffitt, “Attention and visual feedback: the bimanual frame of reference,” in Proc. 1997 Symp. on Interactive 3D Graphics, pp. 121-126.

[39] C. Latulipe, C. S. Kaplan, and C. L. A. Clarke, "Bimanual and unimanual image alignment: an evaluation of mouse-based techniques," in Proc. 18th Annu. ACM Symp. on User Interface Software and Technology, 2005, pp. 123-131.

[40] K. Kin, M. Agrawala, and T. D. DeRose, "Determining the benefits of direct-touch, bimanual, and multifinger input on a multitouch workstation," in Proc. Graphics interface, 2009, pp. 119-124.

[41] X. Xia, P. Irani, and J. Wang, "Evaluation of guiard's theory of bimanual control for navigation and selection," in Proceedings of the 2007 international conference on Ergonomics and health aspects of work with computers, 2007, pp. 368-377.

[42] N. K. Vøllestad, "Measurement of human muscle fatigue," Journal of Neuroscience Methods, vol. 74, no. 2, pp. 219-227, Jun. 1997.

[43] J. R. Cram, “The history of surface electromyography.," Applied Psychophysiology and Biofeedback, vol. 28, no. 2, pp. 81-91, Jun. 2003.

[44] C. J. De Luca, "The use of surface electromyography in biomechanics," Journal of Applied Biomechanics, vol. 13, no. 2, pp. 135-163, 1997.

[45] E. Criswell, Cram's Introduction to Surface Electromyography. Jones and Bartlett, 2011.

[46] G. L. Soderberg, Ed., Selected Topics in Suface Electromyography for Use in the Occupational Setting: Expert Perspectives, vol. 24, no. 2. US Dept. of Health and Human Services, Public Health Service, Centers for Disease Control, National Institute for Occupational Safety and Health, 1993, p. 189.

[47] G. Robertson, G. Caldwell, J. Hamill, G. Kamen, and S. N. Whittlesey, Research Methods in Biomechanics. Human Kinetics, 2004, p. 320. 
[48] O. Lippold, "The relation between integrated action potentials in a human muscle and its isometric tension," The Journal of Physiology, vol. 117, no. 4, pp. 492-499, 1952.

[49] R. Close, "Dynamic properties of mammalian skeletal muscles," Physiological Reviews, vol. 52, no. 1, pp. 129-197, 1972.

[50] E. Kuroda, V. Klissouras, and J. Milsum, "Electrical and metabolic activities and fatigue in human isometric contraction," Journal of Applied Physiology, vol. 29, no. 3, pp. 358-367, 1970 .

[51] J. H. Lawrence and C. De Luca, "Myoelectric signal versus force relationship in different human muscles," Journal of Applied Physiology, vol. 54, no. 6, pp. 1653-1659, 1983.

[52] H. Milner-Brown and R. Stein, "The relation between the surface electromyogram and muscular force.," The Journal of Physiology, vol. 246, no. 3, pp. 549-569, 1975.

[53] Y.Z. Arslan, M. A. Adli, and A. Akan, Investigation of the Relationship Between EMG Signals and the Force Applied to Human Arms. Bursa, Turkey: , 2005.

[54] J. Perry and G. A. Bekey, "EMG-force relationships in skeletal muscle," Critical Reviews in Biomedical Engineering, vol. 7, no. 1, pp. 1-22, 1981.

[55] M. Agarabi, P. Bonato, and C. J. De Luca, "A sEMG-based method for assessing the design of computer mice," in Proc. 26th Annu. Conf. on Engineering in Medicine and Biology Society, 2004, vol. 4, pp. 2450-3.

[56] R. Riemer, A. Ronen, H. Stern, and Y. Edan, "Evaluation of physical stress during hand gesture for human machine interaction," asbweb.org. pp. 1-2, 2008.

[57] S. Cobb and A. Forbes, "Electromyographic studies of muscular fatigue in man," American Journal of Physiology, vol. 65, no. 2, pp. 234-251, 1923.

[58] M. Cifrek, V. Medved, S. Tonković, and S. Ostojić, "Surface EMG based muscle fatigue evaluation in biomechanics.," Clinical Biomechanics, vol. 24, no. 4, pp. 327-340, May. 2009.

[59] H. Niu, R. Li, G. Liu, F. Pu, D. Li, and Y. Fan, "Using EMG to evaluate muscular fatigue induced during video display terminal keyboard use task," in Proc. 7th Asian-Pacific Conf. on Medical and Biological Engineering, 2008, pp. 329-332.

[60] W. Yao, R. J. Fuglevand, and R. M. Enoka, "Motor-unit synchronization increases EMG amplitude and decreases force steadiness of simulated contractions," Journal of Neurophysiology, vol. 83, no. 1, pp. 441-452, 2000.

[61] N. A. Dimitrova and G. V. Dimitrov, "Interpretation of EMG changes with fatigue: facts, pitfalls, and fallacies," Journal of Electromyography and Kinesiology, vol. 13, no. 1, pp. 13-36, Feb. 2003.

[62] L. Lindström, R. Kadefors, and I. Petersén, “An electromyographic index for localized muscle fatigue," Journal of Applied Physiology, vol. 43, no. 4, pp. 750-754, Oct. 1977.

[63] E. Kwatny, D. H. Thomas, and H. G. Kwatny, "An application of signal processing techniques to the study of myoelectric signals.," IEEE Transactions on Biomedical Engineering, vol. 17, no. 4 , pp. 303-313, Oct. 1970.

[64] F. B. Stulen and C. J. De Luca, "The relation between the myoelectric signal and physiological properties of constant-force isometric contractions.," Electroencephalography and Clinical Neurophysiology, vol. 45, no. 6, pp. 681-698, Dec. 1978. 
[65] D. A. Gabriel, S. M. Lester, S. A. Lenhardt, and E. D. J. Cambridge, “Analysis of surface EMG spike shape across different levels of isometric force.," Journal of Neuroscience Methods, vol. 159, no. 1, pp. 146-152, Jan. 2007.

[66] P. Bonato, G. Gagliati, and M. Knaflitz, "Analysis of myoelectric signals recorded during dynamic contractions,” IEEE Engineering in Medicine and Biology Magazine, vol. 15, no. 6, pp. 102-111, 1996.

[67] S. Karlsson, J. Yu, and M. Akay, "Time-frequency analysis of myoelectric signals during dynamic contractions: a comparative study.," IEEE Transactions on Biomedical Engineering, vol. 47 , no. 2, pp. 228-238, Feb. 2000.

[68] D. Farina, R. Merletti, M. Nazzaro, and I. Caruso, "Effect of joint angle on EMG variables in leg and thigh muscles," IEEE Engineering in Medicine and Biology Magazine, vol. 20, no. 6, pp. 62-71, 2001.

[69] C. Lozano, D. Jindrich, and K. Kahol, "The impact on musculoskeletal system during multitouch tablet interactions," in Proc. SIGCHI Conf. on Human Factors in Computing Systems, 2011, pp. 825-828.

[70] A. Murata, A. Uetake, S. Matsumoto, and Y. Takasawa, "Evaluation of shoulder muscular fatigue induced during VDT tasks," International Journal of Human-Computer Interaction, vol. 15 , no. 3, pp. 407-417, Jun. 2003.

[71] S. C. Peres, V. Nguyen, P. T. Kortum, M. Akladios, S. B. Wood, and A. Muddimer,, "Geophysical Software Ergonomics: Objective Measures for Evaluation," unpublished.

[72] S. C. Peres, V. Nguyen, P. T. Kortum, M. Akladios, S. B. Wood, and A. Muddimer, "Software ergonomics: relating subjective and objective measures," in Proc. 27th Int. Conf. Extended Abstracts on Human Factors in Computing Systems, 2009, pp. 3949-3954.

[73] A. DiDomenico and M. A. Nussbaum, "Interactive effects of physical and mental workload on subjective workload assessment," International Journal of Industrial Ergonomics, vol. 38, no. 11-12, pp. 977-983, Nov. 2008.

[74] J. Annett, "Subjective rating scales: science or art," Ergonomics, vol. 45, no. 14, pp. 966-988, 2002.

[75] D. D. Price, P. A. McGrath, A. Rafii, and B. Buckingham, "The validation of visual analogue scales as ratio scale measures for chronic and experimental pain.," Pain, vol. 17, no. 1, pp. 4556, Sep. 1983.

[76] A. K. Zealley and R. C. B. Aitken, "Measurement of mood," Proceedings of the Royal Society of Medicine, vol. 62, no. October, pp. 21-24, 1969.

[77] B. Gupta, "Ergonomic soft mouse and armrest mouse pad," Ubiquity, 2004.

[78] P. L. Weiss, C. P. Whiteley, J. Treviranus, and D. I. Fels, "PEBBLES: A Personal Technology for Meeting Educational, Social and Emotional Needs of Hospitalised Children," Personal and Ubiquitous Computing, vol. 5, no. 3, pp. 157-168, Aug. 2001.

[79] A. Belz and E. Kow, "Discrete vs. continuous rating scales for language evaluation in NLP," in Proc. 49th Annu. Meeting of the Association for Computational Linguistics: Human Language Technologies, 2011, pp. 230-235. 
[80] G. Borg, P. Hassmén, and M. Lagerström, "Perceived exertion related to heart rate and blood lactate during arm and leg exercise.," European journal of applied physiology and occupational physiology, vol. 56, no. 6, pp. 679-85, Jan. 1987.

[81] G. Borg, Physical performance and perceived exertion. C. W. K. Gleerup, 1962, p. 64.

[82] G. Borg, Borg's perceived exertion and pain scales. Human Kinetics, 1998, p. 104.

[83] G. Borg and E. Borg, "A new generation of scaling methods: Level-anchored ratio scaling," Psychologica, vol. 28, pp. 15-45, 2001.

[84] J. F. Knight, D. Williams, T. N. Arvanities, B. Chris, A. Wichmann, M. Wittkaemper, I. Herbst, and S. Sotiriou, "Wearability assessment of a mobile augmented reality system," in Proc. 11th Int. Conf. on Virtual Systems and MultiMedia, 2005.

[85] T. N. Arvanitis, A. Petrou, J. F. Knight, S. Savas, S. Sotiriou, M. Gargalakos, and E. Gialouri, "Human factors and qualitative pedagogical evaluation of a mobile augmented reality system for science education used by learners with physical disabilities," Personal and Ubiquitous Computing, vol. 13, no. 3, pp. 243-250, Nov. 2009.

[86] P. Weller, L. Rakhmetova, Q. Ma, and G. Mandersloot, "Evaluation of a wearable computer system for telemonitoring in a critical environment," Personal and Ubiquitous Computing, vol. 14, no. 1, pp. 73-81, Apr. 2009.

[87] G. Neely, G. Ljunggren, C. Sylven, and G. A. V. Borg, "Comparison between the Visual Analogue Scale (VAS) and the Category Ratio Scale (CR-10) for the evaluation of leg exertion," International Journal of Sports Medicine, vol. 13, no. 2, pp. 133-136, 1992.

[88] S. Grant, "A Comparison of the reproducibility and the sensitivity to change of visual analogue scales, Borg scales, and Likert scales in normal subjects during submaximal exercise," Chest, vol. 116, no. 5, pp. 1208-1217, Nov. 1999.

[89] E. M. Capodaglio, "Comparison between the CR10 Borg's scale and the VAS (visual analogue scale) during an arm-cranking exercise," Journal of Occupational Rehabilitation, vol. 11, no. 2, pp. 69-74, 2001.

[90] E. Borg and L. Kaijser, "A comparison between three rating scales for perceived exertion and two different work tests," Scandinavian Journal of Medicine \& Science in Sports, vol. 16, no. 1, pp. 57-69, Feb. 2006.

[91] S. G. Hart and L. E. Staveland, "Development of NASA-TLX (task load index): results of empirical and theoretical research," in Human Mental Workload, vol. 52, P. Hancock and N. Meshkati, Eds. Amsterdam, Holland: Elsevier Science Publishers, 1988, pp. 139-183.

[92] R. Shively, V. Battiste, J. H. Matsumoto, D. D. Pepitone, M. R. Bortolussi, and S. G. Hart, "Inflight evaluation of pilot workload measures for rotorcraft research," in Proc. 4th Int. Symp. on Aviation Psychology, 1987, pp. 637-643.

[93] T. Horberry, J. Anderson, M. a Regan, T. J. Triggs, and J. Brown, "Driver distraction: the effects of concurrent in-vehicle tasks, road environment complexity and age on driving performance.," Accident Analysis and Prevention, vol. 38, pp. 185-191, Jan. 2006.

[94] R. D. Stevens and A. D. N. Edwards, "An approach to the evaluation of assistive technology," in Proc. 2nd ACM Conf. on Assistive technologies, 1996, pp. 64-71. 
[95] K. Seewoonauth, E. Rukzio, R. Hardy, and P. Holleis, "Touch \& connect and touch \& select: interacting with a computer by touching it with a mobile phone," in Proc. 11th Int. Conf. on Human-Computer Interaction with Mobile Devices and Services, 2009, pp. 1-9.

[96] G. Shoemaker, L. Findlater, J. Q. Dawson, and K. S. Booth, "Mid-air text input techniques for very large wall displays," in Proceedings of Graphics Interface 2009, 2009, pp. 231-238.

[97] E. W. Pedersen and K. Hornbæk, "Tangible bots: interaction with active tangibles in tabletop interfaces," in Proc. SIGCHI Conf. on Human Factors in Computing Systems, 2011, pp. 29752984.

[98] M. A. Vidulich and P. S. Tsang, "Assessing subjective workload assessment: A comparison of SWAT and the NASA-Bipolar methods," Human Factors and Ergonomics Society Annual Meeting, vol. 29, no. 1, pp. 71-75, 1985.

[99] S. G. Hill, H. P. Iavecchia, J. C. Byers, A. C. Bittner, A. L. Zaklad, and R. E. Christ, "Comparison of four subjective workload rating scales," The Journal of the Human Factors and Ergonomics Society, vol. 34, no. 4, pp. 429-439, 1992.

[100] S. Rubio, E. Diaz, J. Martin, and J. M. Puente, "Evaluation of subjective mental workload: a comparison of SWAT, NASA-TLX, and workload profile methods," Applied Psychology, vol. 53, no. 1, pp. 61-86, Jan. 2004.

[101] Microsoft, "Microsoft Surface Fact Sheet," 2007. [Online]. Available: http://www.microsoft.com/presspass/presskits/surfacecomputing/docs/MSSurface2008FS.doc. [Accessed: 18-Jul-2011].

[102] Noraxon, “ZeroWire.” [Online]. Available: http://www.noraxon.com/products/zerowire.php. [Accessed: 16-Jul-2011].

[103] Microsoft, "Surface Toolkit for Windows Touch Beta," 2011. [Online]. Available: http://msdn.microsoft.com/en-us/library/ee957351(v=Surface.15).aspx. [Accessed: 18-Jun2011].

[104] NISMAT, "Biomechanics et al. Toolbox (BEAT).” [Online]. Available: http://www.nismat.org/research/software/beat/index.html. [Accessed: 10-Jul-2011]. 


\section{Appendix A}

\section{Experiment Materials}

\section{A.1 Isometric Contraction Electromyogram Analysis}

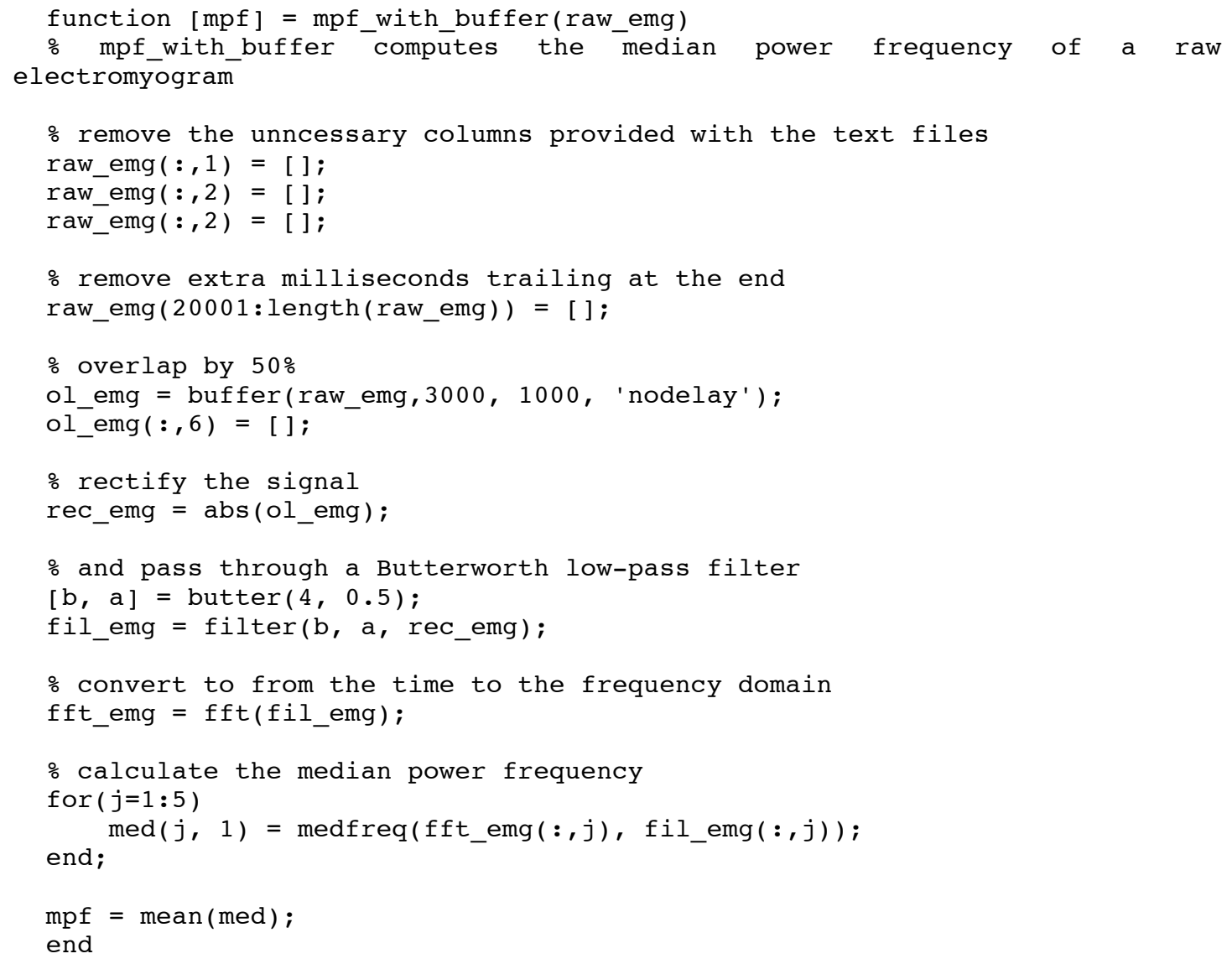

\section{A.2 Electromyogram Analysis}

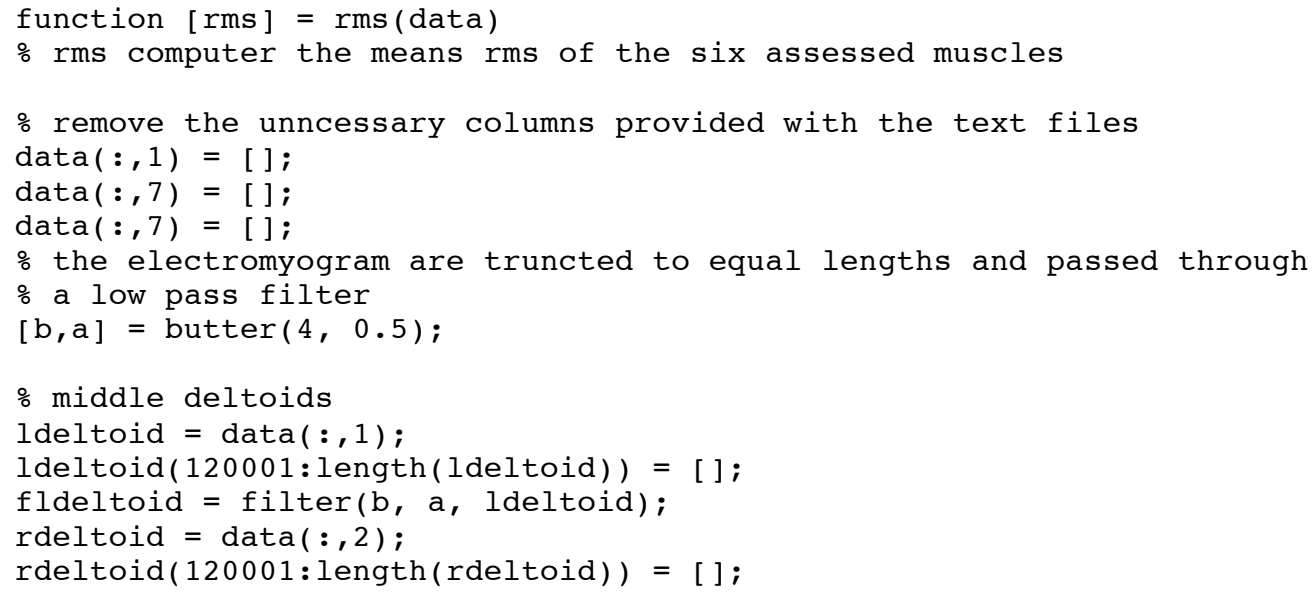




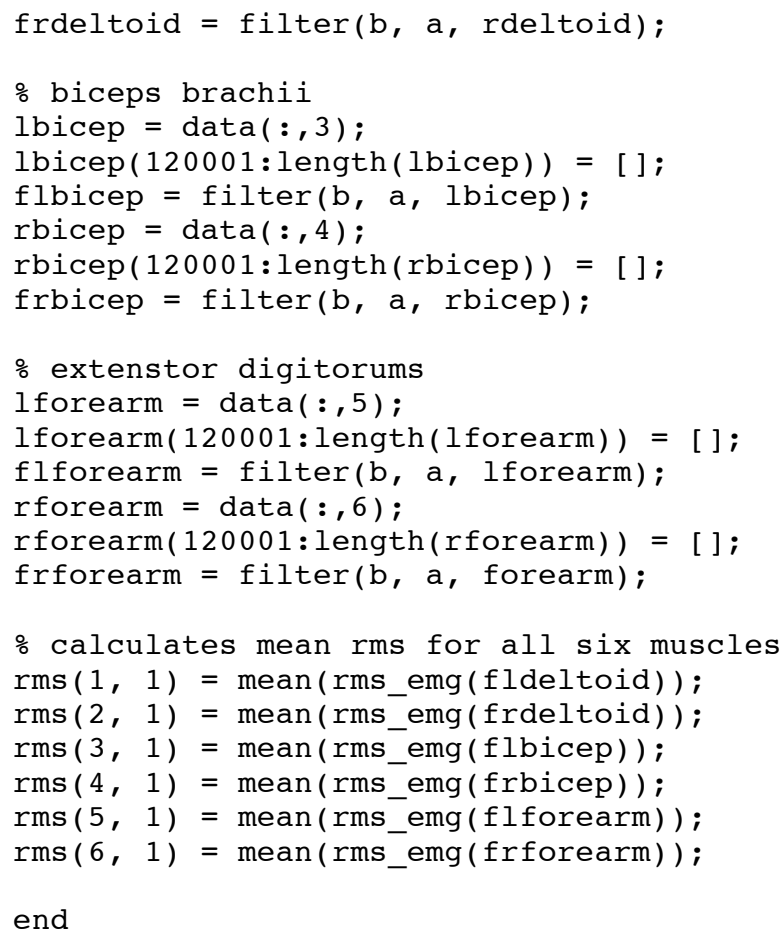




\section{Appendix B}

\section{Experiment Results}

\section{B.1 Subjective Ratings Results}

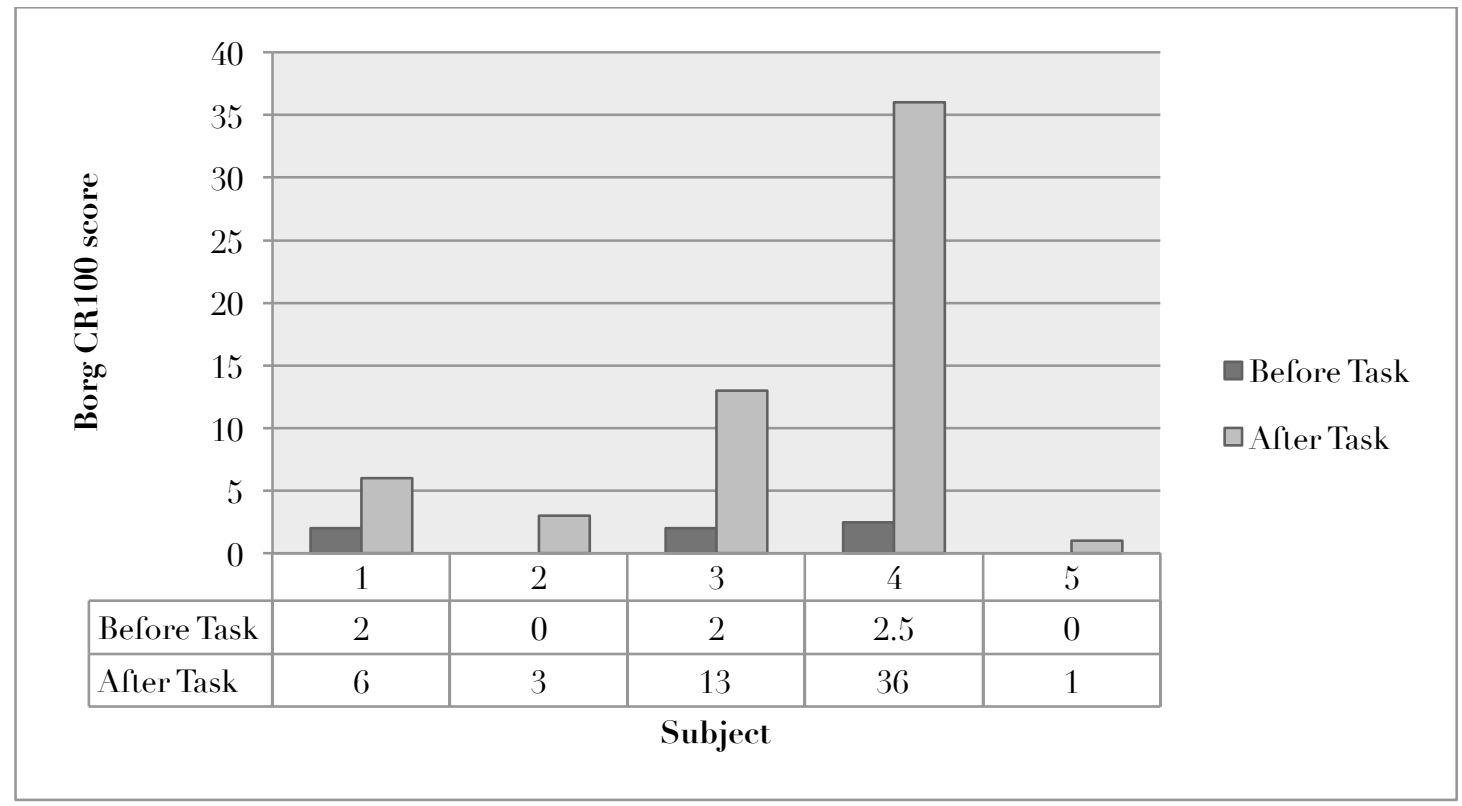

Figure B-1: the subjective ratings collected before and after the experimental task for the horizontal condition

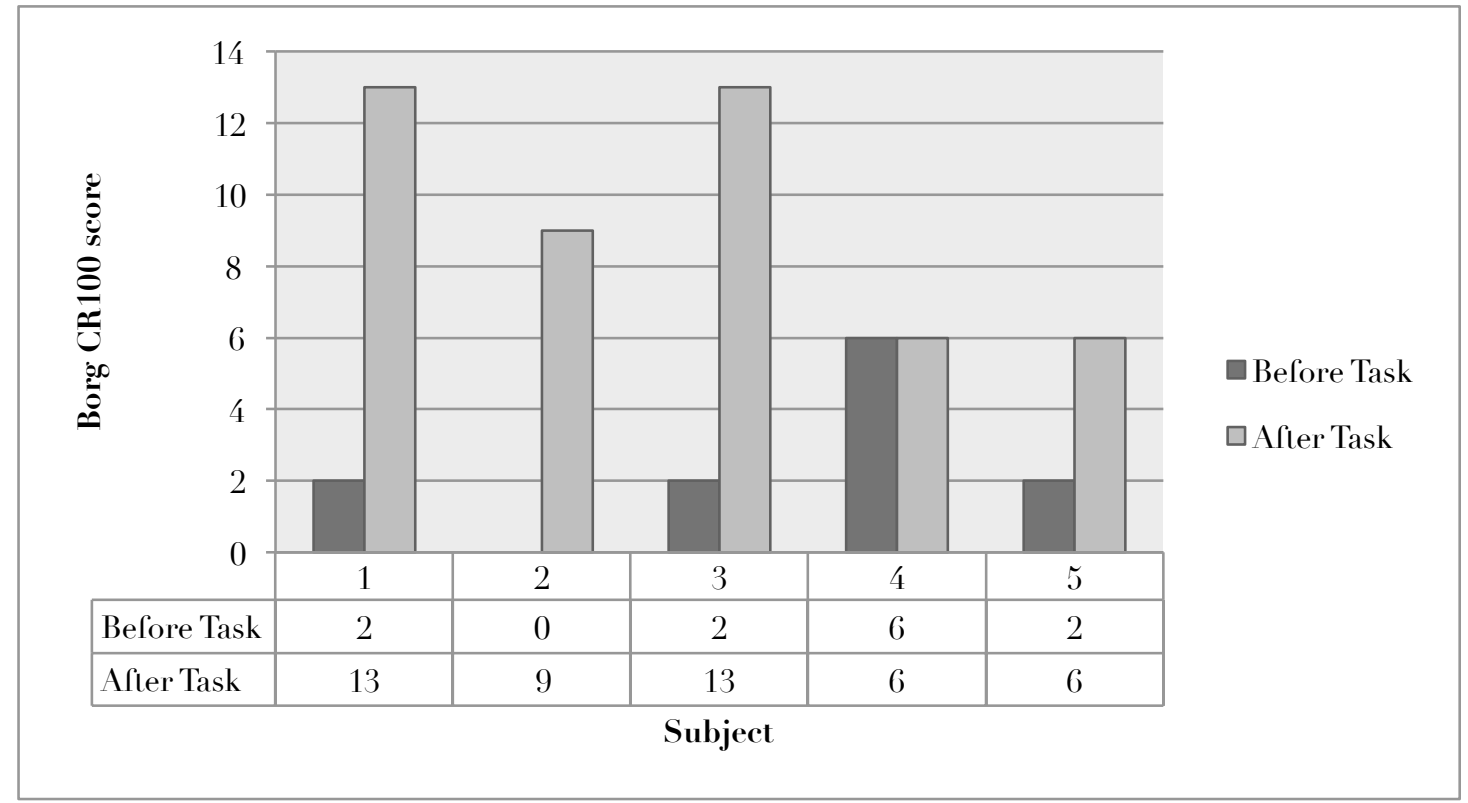

Figure B-2: the subjective ratings collected before and after the experimental task for the vertical condition 


\section{B.2 Isometric Contraction Electromyogram Results}

\section{B.2.1 Isometric Contraction Results for the Horizontal Condition}

\begin{tabular}{lrr}
\hline & Variable 1 & Variable 2 \\
\hline Mean & 11.72028 & 16.9975 \\
Variance & 33.09534711 & 58.88441636 \\
Observations & 5 & 5 \\
Pearson Correlation & 0.614688717 & \\
Hypothesized Mean Difference & 0 & \\
df & 4 & \\
$\mathbf{t}$ Stat & -1.92163025 & \\
$\mathbf{P}(\mathbf{T}<=\mathbf{t})$ one-tail & 0.06351644 & \\
$\mathbf{t}$ Critical one-tail & 2.131846782 & \\
\hline
\end{tabular}

Table B-1: paired t-test results for the left middle deltoid isometric contraction pre- and post-recordings

\begin{tabular}{lrr}
\hline & Variable 1 & Variable 2 \\
\hline Mean & 15.46232 & 25.34684 \\
Variance & 49.46435246 & 468.8330389 \\
Observations & 5 & 5 \\
Pearson Correlation & 0.930642285 & \\
Hypothesized Mean Difference & 0 & \\
df & 4 & \\
t Stat & -1.442256942 & \\
P $(\mathbf{T}<=\mathbf{t})$ one-tail & 0.111346106 & \\
$\mathbf{t}$ Critical one-tail & 2.131846782 & \\
\hline
\end{tabular}

Table B-2: paired t-test results for the right middle deltoid isometric contraction pre- and post-recordings

\begin{tabular}{lrr}
\hline & Variable 1 & Variable 2 \\
\hline Mean & 6.14014 & 6.84456 \\
Variance & 5.585563253 & 21.71412757 \\
Observations & 5 & 5 \\
Pearson Correlation & 0.906983547 & \\
Hypothesized Mean Difference & 0 & \\
df & 4 & \\
t Stat & -0.58208517 & \\
$\mathbf{P}(\mathbf{T}<=\mathbf{t})$ one-tail & 0.295869804 & \\
$\mathbf{t}$ Critical one-tail & 2.131846782 & \\
\hline
\end{tabular}

Table B-3: paired t-test results for the left biceps brachii isometric contraction pre- and post-recordings 


\begin{tabular}{lrr}
\hline & Variable 1 & Variable 2 \\
\hline Mean & 7.25332 & 8.3457 \\
Variance & 7.736647817 & 51.56615462 \\
Observations & 5 & 5 \\
Pearson Correlation & -0.122059429 & \\
Hypothesized Mean Difference & 0 & \\
df & 4 & \\
t Stat & -0.304903966 & \\
P $(\mathbf{T}<=\mathbf{t})$ one-tail & 0.387822802 & \\
$\mathbf{t}$ Critical one-tail & 2.131846782 & \\
\hline
\end{tabular}

Table B-4: paired t-test results for the right biceps brachii isometric contraction pre- and post-recordings

\begin{tabular}{lrr}
\hline & Variable 1 & Variable 2 \\
\hline Mean & 12.23784 & 11.92964 \\
Variance & 108.6942869 & 25.23346963 \\
Observations & 5 & 5 \\
Pearson Correlation & 0.594662407 & \\
Hypothesized Mean Difference & 0 & \\
df & 4 & \\
t Stat & 0.081420796 \\
P $(\mathbf{T}<=\mathbf{t})$ one-tail & 0.469509298 \\
t Critical one-tail & 2.131846782 & \\
\hline
\end{tabular}

Table B-5: paired t-test results for the left extensor digitorum isometric contraction pre- and post-recordings

\begin{tabular}{lrr}
\hline & Variable 1 & Variable 2 \\
\hline Mean & 8.58258 & 6.64932 \\
Variance & 5.183230277 & 4.703241547 \\
Observations & 5 & 5 \\
Pearson Correlation & -0.187891206 & \\
Hypothesized Mean Difference & 0 & \\
df & \multicolumn{1}{c}{4} \\
t Stat & 1.2615567 & \\
P $(\mathbf{T}<=\mathbf{t})$ one-tail & 0.137831745 & \\
t Critical one-tail & 2.131846782 & \\
\hline
\end{tabular}

Table B-6: paired t-test results for the right extensor digitorum isometric contraction pre- and post-recordings 


\section{B.2.2 Isometric Contraction Results for the Vertical Condition}

\begin{tabular}{lrr}
\hline & Variable 1 & Variable 2 \\
\hline Mean & 23.64928333 & 18.2009 \\
Variance & 249.1550426 & 205.0437084 \\
Observations & 6 & 6 \\
Pearson Correlation & 0.812368312 & \\
Hypothesized Mean Difference & 0 & \\
df & 5 & \\
$\mathbf{t}$ Stat & 1.431092646 & \\
$\mathbf{P}(\mathbf{T}<=\mathbf{t})$ one-tail & 0.105907318 & \\
$\mathbf{t}$ Critical one-tail & 2.015048372 & \\
\hline
\end{tabular}

Table B-7: paired t-test results for the left middle deltoid isometric contraction pre- and post-recordings

\begin{tabular}{lrr}
\hline & Variable 1 & Variable 2 \\
\hline Mean & 36.00811667 & 28.3754 \\
Variance & 967.5473717 & 547.458394 \\
Observations & 6 & 6 \\
Pearson Correlation & 0.853399827 & \\
Hypothesized Mean Difference & 0 & \\
df & 5 & \\
t Stat & 1.131967976 & \\
P $(\mathbf{T}<=\mathbf{t})$ one-tail & 0.154498329 & \\
t Critical one-tail & 2.015048372 & \\
\hline
\end{tabular}

Table B-8: paired t-test results for the right middle deltoid isometric contraction pre- and post-recordings

\begin{tabular}{lrr}
\hline & Variable 1 & Variable 2 \\
\hline Mean & 4.0381 & 4.413166667 \\
Variance & 3.567690452 & 7.061511631 \\
Observations & 6 & 6 \\
Pearson Correlation & -0.022647308 & \\
Hypothesized Mean Difference & 0 & \\
df & 5 & \\
t Stat & -0.278829164 & \\
$\mathbf{P}(\mathbf{T}<=\mathbf{t})$ one-tail & 0.395770239 & \\
$\mathbf{t}$ Critical one-tail & 2.015048372 & \\
\hline
\end{tabular}

Table B-9: paired t-test results for the left biceps brachii isometric contraction pre- and post-recordings 


\begin{tabular}{lrr}
\hline & Variable 1 & Variable 2 \\
\hline Mean & 6.46415 & 5.524233333 \\
Variance & 25.5970761 & 12.42421007 \\
Observations & 6 & 6 \\
Pearson Correlation & 0.919119291 & \\
Hypothesized Mean Difference & 0 & \\
df & 5 & \\
t Stat & 1.005811077 & \\
P $(\mathbf{T}<=\mathbf{t})$ one-tail & 0.180335864 & \\
t Critical one-tail & 2.015048372 & \\
\hline
\end{tabular}

Table B-10: paired t-test results for the right biceps brachii isometric contraction pre- and post-recordings

\begin{tabular}{lrr}
\hline & Variable 1 & Variable 2 \\
\hline Mean & 11.5535 & 11.3467 \\
Variance & 49.51029404 & 7.408871308 \\
Observations & 6 & 6 \\
Pearson Correlation & 0.845893507 & \\
Hypothesized Mean Difference & 0 & \\
df & 5 & \\
t Stat & 0.102303261 & \\
P $(\mathbf{T}<=\mathbf{t})$ one-tail & 0.461246083 & \\
t Critical one-tail & 2.015048372 & \\
\hline
\end{tabular}

Table B-11: paired t-test results for the left extensor digitorum isometric contraction pre- and post-recordings

\begin{tabular}{lrr}
\hline & Variable 1 & Variable 2 \\
\hline Mean & 9.647366667 & 11.06596667 \\
Variance & 26.39314935 & 43.93118113 \\
Observations & 6 & 6 \\
Pearson Correlation & 0.908572972 & \\
Hypothesized Mean Difference & 0 & \\
df & 5 & \\
t Stat & -1.19549775 & \\
P $(\mathbf{T}<=\mathbf{t})$ one-tail & 0.142747407 & \\
$\mathbf{t}$ Critical one-tail & 2.015048372 & \\
\hline
\end{tabular}

Table B-12: paired t-test results for the right extensor digitorum isometric contraction pre- and post-recordings 


\section{B.3 Electromyogram ANOVA Results}

\section{B.3.1 Electromyogram ANOVA Results for the Horizontal Condition}

\begin{tabular}{|c|c|c|c|c|c|c|}
\hline $\begin{array}{l}\text { Source of } \\
\text { Variation }\end{array}$ & $S S$ & $d f$ & $M S$ & $F$ & P-value & $F$ crit \\
\hline Rows & 2316.728073 & 4 & 579.1820183 & 33.82057013 & $9.74863 \mathrm{E}-12$ & 2.633532094 \\
\hline Columns & 248.5406852 & 9 & 27.61563169 & 1.612578393 & 0.148724854 & 2.152607472 \\
\hline Error & 616.5050612 & 36 & 17.12514059 & & & \\
\hline Total & 3181.77382 & 49 & & & & \\
\hline
\end{tabular}

Table B-13: ANOVA results for the left middle deltoid electromyograms recorded during the task

\begin{tabular}{|c|c|c|c|c|c|c|}
\hline $\begin{array}{l}\text { Source of } \\
\text { Variation }\end{array}$ & $S S$ & $d f$ & $M S$ & $F$ & P-value & $F$ crit \\
\hline Rows & 7398.257335 & 4 & 1849.564334 & 14.11920825 & $5.05561 \mathrm{E}-07$ & 2.633532094 \\
\hline Columns & 765.3335951 & 9 & 85.03706612 & 0.649156141 & 0.747736645 & 2.152607472 \\
\hline Error & 4715.867551 & 36 & 130.9963209 & & & \\
\hline Total & 12879.45848 & 49 & & & & \\
\hline
\end{tabular}

Table B-14: ANOVA results for the right middle deltoid electromyograms recorded during the task

\begin{tabular}{|c|c|c|c|c|c|c|}
\hline $\begin{array}{l}\text { Source of } \\
\text { Variation } \\
\end{array}$ & $S S$ & $d f$ & $M S$ & $F$ & P-value & $F$ crit \\
\hline Rows & 1666.365815 & 4 & 416.5914538 & 15.8501284 & $1.43449 \mathrm{E}-07$ & 2.633532094 \\
\hline Columns & 305.4541141 & 9 & 33.93934601 & 1.291296274 & 0.275346483 & 2.152607472 \\
\hline Error & 946.1937443 & 36 & 26.28315956 & & & \\
\hline Total & 2918.013673 & 49 & & & & \\
\hline
\end{tabular}

Table B-15: ANOVA results for the left biceps brachii electromyograms recorded during the task

\begin{tabular}{|c|c|c|c|c|c|c|}
\hline $\begin{array}{l}\text { Source of } \\
\text { Variation }\end{array}$ & $S S$ & $d f$ & $M S$ & $F$ & P-value & $F$ crit \\
\hline Rows & 2694.438572 & 4 & 673.6096429 & 16.07918137 & $1.22191 \mathrm{E}-07$ & 2.633532094 \\
\hline Columns & 327.7103261 & 9 & 36.41225845 & 0.869167052 & 0.560591772 & 2.152607472 \\
\hline Error & 1508.15807 & 36 & 41.89327973 & & & \\
\hline Total & 4530.306968 & 49 & & & & \\
\hline
\end{tabular}

Table B-16: ANOVA results for the right biceps brachii electromyograms recorded during the task 


\begin{tabular}{lcrrrrr}
\hline \multicolumn{1}{c}{$\begin{array}{c}\text { Source of } \\
\text { Variation }\end{array}$} & \multicolumn{1}{c}{$S S$} & $d f$ & \multicolumn{1}{c}{$M S$} & \multicolumn{1}{c}{$\boldsymbol{F}$} & P-salue & F crit \\
\hline Rows & 21862.81191 & 4 & 5465.702978 & 53.12676821 & $1.29417 \mathrm{E}-14$ & 2.633532094 \\
Columns & 889.9156233 & 9 & 98.8795137 & 0.96111132 & 0.487103531 & 2.152607472 \\
Error & 3703.694274 & 36 & 102.8803965 & & & \\
& & & & & & \\
Total & 26456.42181 & 49 & & & & \\
\hline
\end{tabular}

Table B-17: ANOVA results for the left extensor digitorum electromyograms recorded during the task

\begin{tabular}{|c|c|c|c|c|c|c|}
\hline $\begin{array}{l}\text { Source of } \\
\text { Variation }\end{array}$ & $S S$ & $d f$ & $M S$ & $F$ & P-value & $F$ crit \\
\hline Rows & 17723.40008 & 4 & 4430.850019 & 51.44151582 & $2.11369 \mathrm{E}-14$ & 2.633532094 \\
\hline Columns & 676.7145015 & 9 & 75.19050017 & 0.872950627 & 0.557472906 & 2.152607472 \\
\hline Error & 3100.814549 & 36 & 86.13373748 & & & \\
\hline Total & 21500.92913 & 49 & & & & \\
\hline
\end{tabular}

Table B-18: ANOVA results for the right extensor digitorum electromyograms recorded during the task

B.3.2 Electromyogram ANOVA Results for the Vertical Condition

\begin{tabular}{|c|c|c|c|c|c|c|}
\hline $\begin{array}{l}\text { Source of } \\
\text { Variation } \\
\end{array}$ & $S S$ & $d f$ & $M S$ & $F$ & P-value & $F$ crit \\
\hline Rows & 18998.87945 & 5 & 3799.775891 & 26.31947706 & $2.49903 \mathrm{E}-12$ & 2.422085459 \\
\hline Columns & 3202.113996 & 9 & 355.790444 & 2.464413349 & 0.022291717 & 2.095755094 \\
\hline Error & 6496.706401 & 45 & 144.3712533 & & & \\
\hline Total & 28697.69985 & 59 & & & & \\
\hline
\end{tabular}

Table B-19: ANOVA results for the left middle deltoid electromyograms recorded during the task

\begin{tabular}{|c|c|c|c|c|c|c|}
\hline $\begin{array}{l}\text { Source of } \\
\text { Variation }\end{array}$ & $S S$ & $d f$ & $M S$ & $F$ & P-salue & $F$ crit \\
\hline Rows & 69840.10855 & 5 & 13968.02171 & 24.09763481 & $1.04327 \mathrm{E}-11$ & 2.422085459 \\
\hline Columns & 6620.489901 & 9 & 735.609989 & 1.269074551 & 0.279819293 & 2.095755094 \\
\hline Error & 26083.92823 & 45 & 579.6428496 & & & \\
\hline Total & 102544.5267 & 59 & & & & \\
\hline
\end{tabular}

Table B-20: ANOVA results for the right middle deltoid electromyograms recorded during the task 


\begin{tabular}{|c|c|c|c|c|c|c|}
\hline $\begin{array}{l}\text { Source of } \\
\text { Variation } \\
\end{array}$ & $S S$ & $d f$ & $M S$ & $F$ & P-value & $F$ crit \\
\hline Rows & 2385.739653 & 5 & 477.1479306 & 34.57259652 & $2.4234 \mathrm{E}-14$ & 2.422085459 \\
\hline Columns & 213.8481006 & 9 & 23.76090007 & 1.721638005 & 0.111857293 & 2.095755094 \\
\hline Error & 621.0600023 & 45 & 13.80133338 & & & \\
\hline Total & 3220.647756 & 59 & & & & \\
\hline
\end{tabular}

Table B-21: ANOVA results for the left biceps brachii electromyograms recorded during the task

\begin{tabular}{|c|c|c|c|c|c|c|}
\hline $\begin{array}{l}\text { Source of } \\
\text { Variation }\end{array}$ & $S S$ & $d f$ & $M S$ & $F$ & P-salue & $F$ crit \\
\hline Rows & 8142.138478 & 5 & 1628.427696 & 46.66151022 & $1.06098 \mathrm{E}-16$ & 2.422085459 \\
\hline Columns & 393.0656817 & 9 & 43.67396464 & 1.251448347 & 0.289358035 & 2.095755094 \\
\hline Error & 1570.44309 & 45 & 34.89873534 & & & \\
\hline Total & 10105.64725 & 59 & & & & \\
\hline
\end{tabular}

Table B-22: ANOVA results for right biceps brachii electromyograms recorded during the task

\begin{tabular}{|c|c|c|c|c|c|c|}
\hline $\begin{array}{l}\text { Source of } \\
\text { Variation }\end{array}$ & $S S$ & $d f$ & $M S$ & $F$ & P-value & $F$ crit \\
\hline Rows & 28192.62601 & 5 & 5638.525202 & 40.230852 & $1.62025 \mathrm{E}-15$ & 2.422085459 \\
\hline Columns & 2130.683725 & 9 & 236.7426362 & 1.689157646 & 0.1197882 & 2.095755094 \\
\hline Error & 6306.9416 & 45 & 140.1542578 & & & \\
\hline Total & 36630.25134 & 59 & & & & \\
\hline
\end{tabular}

Table B-23: ANOVA results for the left extensor digitorum electromyograms recorded during the task

\begin{tabular}{|c|c|c|c|c|c|c|}
\hline $\begin{array}{l}\text { Source of } \\
\text { Variation } \\
\end{array}$ & $S S$ & $d f$ & $M S$ & $F$ & P-value & $F$ crit \\
\hline Rows & 23896.66452 & 5 & 4779.332903 & 33.57670368 & 4.04195E-14 & 2.422085459 \\
\hline Columns & 915.8332133 & 9 & 101.7592459 & 0.714898944 & 0.692377958 & 2.095755094 \\
\hline Error & 6405.333374 & 45 & 142.3407416 & & & \\
\hline Total & 31217.8311 & 59 & & & & \\
\hline
\end{tabular}

Table B-24: ANOVA results for the right extensor digitorum electromyograms recorded during the task 


\section{B.4 Video Results}

\begin{tabular}{|c|c|}
\hline \multicolumn{2}{|r|}{ Horizontal } \\
\hline \multicolumn{2}{|r|}{ Subject 1} \\
\hline 1 from $1-5$ & $\begin{array}{ll}\text { - } & \text { Unimanual multi-finger translation } \\
\text { - } & \text { Unimanual multi-finger rotation } \\
\text { - } & \text { Bimanual multi-finger scaling if piece very large } \\
\text { - } & \text { Bimanual one-finger scaling if pieces normal or small } \\
\end{array}$ \\
\hline 2 from $6-11$ & $\begin{array}{l}\text { - } \quad \text { Behavior from } 1 \text { maintained } \\
\text { A piece was bought to the left resting palm to be scaled one-finger } \\
\text { bimanually }\end{array}$ \\
\hline 3 from $12-17$ & $\begin{array}{l}\text { - } \text { Behavior changed from } 2 \\
\text { - Left hand resting with unimanual multi-finger or two finger (index } \\
\text { and thumb) scaling with the right hand } \\
\text { - Also rotation unimanual multi-finger or two finger (index and } \\
\text { thumb) } \\
\text { - Used bimanual scaling and rotation occasionally } \\
\text { Unimanual one-finger translation }\end{array}$ \\
\hline 4 from $18-23$ & $\begin{array}{ll}\text { - } & \text { Behavior from } 3 \text { maintained } \\
\text { - } & \text { Sat sideways } \\
\text { - } & \text { Unimanual scaling with two finger }(2 \text { and } 3+\text { was noted once } \\
& \text { to match two pieces together }\end{array}$ \\
\hline 5 from $24-29$ & $\begin{array}{l}\text { - } \quad \text { Behavior from } 4 \text { maintained } \\
\text { - Hand was occasionally rested on lap } \\
\text { At the end, rested right hand and used left hand unimanually one- } \\
\text { finger to translate }\end{array}$ \\
\hline 6 from $30-35$ & $\begin{array}{l}\text { - } \text { Behavior changed from } 5 \\
\text { More bimanual one-finger scaling and multi-finger and also one- } \\
\text { finger rotation } \\
\text { As right hand rested, left hand was used for translating } \\
\text { unimanually one-finger } \\
\text { After while, right hand was used unimanually }\end{array}$ \\
\hline 7 from $36-41$ & $\begin{array}{ll}\text { - } & \text { Behavior from } 6 \text { maintained } \\
\text { Alternated more between left and right hand for translations to be } \\
\text { used unimanually with one-finger }\end{array}$ \\
\hline 8 from $42-47$ & $\begin{array}{ll} & \text { Behavior from } 7 \text { maintained } \\
\text { - } & \text { Wiped hand with clothes when piece won't move }\end{array}$ \\
\hline 9 from $48-53$ & - $\quad$ Behavior from 8 maintained \\
\hline 10 from 54-59 & $\begin{array}{l}\text { - } \quad \text { Behavior from } 9 \text { changed } \\
\text { - } \quad \text { Unimanual one-finger right hand translation } \\
\text { - } \quad \text { Nimanual one-finger scaling } \\
\text { - No arm resting, but palm resting when left hand not used }\end{array}$ \\
\hline \multicolumn{2}{|r|}{ Subject 2} \\
\hline 1 from $1-5$ & $\begin{array}{l}\text { - Unimanual one-finger translation } \\
\text { - Unimanual two finger (index and thumb) scaling } \\
\text { - } \quad \text { Alternated arms and used whichever closest to piece, when not } \\
\text { - used resting on lap } \\
\text { - Unimanual two finger (index and thumb) rotation } \\
\text { Unimanual multi-finger noted for bigger pieces }\end{array}$ \\
\hline 2 from $6-11$ & $\begin{array}{l}\text { - } \quad \text { Behavior from } 1 \text { maintained } \\
\text { EXCEPT at } 9 \mathrm{~min} \text { where bimanual one-finger scaling was used } \\
\text { occasionally and only noted for bigger pieces }\end{array}$ \\
\hline 3 from $12-17$ & $\begin{array}{ll}\text { - } & \text { Behavior from } 2 \text { maintained } \\
\text { - } & \text { Also unimanual multi-finger translation noted }\end{array}$ \\
\hline
\end{tabular}




\begin{tabular}{|c|c|}
\hline 4 from $18-23$ & $\begin{array}{ll}\cdot & \text { Behavior from } 3 \text { maintained } \\
- & \text { Also noted unimanual } 3 \text { finger scaling }(2-3, \text {, and thumb) } \\
\text { - } & \text { And Unimanual } 2 \text { finger scaling }\left(2-\text { and } 3^{-}\right)\end{array}$ \\
\hline 5 from $24-29$ & $\begin{array}{l}\text { - } \quad \text { Behavior from } 4 \text { maintained } \\
\text { - } \quad \text { One instance of bimanual rotation with one-finger from each }\end{array}$ \\
\hline 6 from $30-35$ & - $\quad$ Behavior from 5 maintained \\
\hline 7 from $36-41$ & $\begin{array}{ll}\text { - } & \text { Behavior from } 6 \text { maintained } \\
\text { - } & \text { Also noted unimanual multi-finger rotation }\end{array}$ \\
\hline 8 from $42-47$ & $\begin{array}{ll}\text { - } & \text { Behavior from } 7 \text { maintained } \\
\text { - } & \text { At the end, rest both arms on table }\end{array}$ \\
\hline 9 from $48-53$ & - $\quad$ Behavior for 8 maintained \\
\hline 10 from $54-59$ & - $\quad$ Behavior from 9 maintained \\
\hline \multicolumn{2}{|r|}{ Subject 3} \\
\hline 1 from $1-5$ & $\begin{array}{ll}\text { - } & \text { Unimanual two finger }\left(2 \text { and } 3^{m}\right) \text { and (index and thumb) scaling } \\
\text { - } & \text { Bimanual one-finger scaling } \\
\text { - } & \text { Unimanual one-finger rotation } \\
& \text { Unimal one-finger translation }\end{array}$ \\
\hline 2 from $6-11$ & $\begin{array}{l}\text { - } \quad \text { Behavior from } 1 \text { maintained } \\
\text { - } \quad \text { More bimanual scaling as the pieces matched into bigger ones }\end{array}$ \\
\hline 3 from 12.17 & $\begin{array}{ll} & \text { Behavior from } 2 \text { maintained }\end{array}$ \\
\hline 4 from $18-23$ & $\begin{array}{ll}\text { - } & \text { Behavior from } 3 \text { maintained } \\
\text { - } & \text { Also unimanual two finger (index and thumb) was noted } \\
\end{array}$ \\
\hline 5 from 24-29 & $\begin{array}{ll}\text { - } & \text { Behavior from } 4 \text { maintained } \\
\text { - } & \text { Also unimanual two finger }(3 " \text { and thumb }) \text { scaling and rotation } \\
\text { - } & \text { Left palm resting on edge of table }\end{array}$ \\
\hline 6 from $30-35$ & - $\quad$ Behavior from 5 maintained \\
\hline 7 from $36-41$ & - $\quad$ Behavior from 6 maintained \\
\hline 8 from $42-47$ & $\begin{array}{l}\text { - } \quad \text { Behavior changed from } 7 \\
\text { - The pieces were bough to the left hand, which was resting on edge, } \\
\text { to be scaled down bimanually one-finger } \\
\text { - Unimanual one-finger translation and rotation }\end{array}$ \\
\hline 9 from $48-53$ & $\begin{array}{l}\text { - Behavior from } 8 \text { changed, now the arm is resting on side of table } \\
\text { and also alternated with palm resting } \\
\text { - Unimanual one-finger translation and rotation } \\
\text { - Bimanual one-finger scaling calls the left hand out of rest } \\
\text { Bimanual one-finger rotation }\end{array}$ \\
\hline 10 from $54-59$ & - $\quad$ Behavior from 9 maintained \\
\hline \multicolumn{2}{|r|}{ Subject 4} \\
\hline 1 from $1-5$ & $\begin{array}{ll}\text { - } & \text { Unimanual one-finger translation } \\
\text { - } & \text { Bimanual one-finger scaling } \\
\text { Bimanual one-finger rotation }\end{array}$ \\
\hline 2 from $6-11$ & $\begin{array}{l}\text { - } \quad \text { Behavior from } 1 \text { maintained } \\
\text { Left arm resting on table (pic) and when needed moved from } \\
\text { resting position }\end{array}$ \\
\hline 3 from $12-17$ & $\begin{array}{l}\text { - Occasional unimanual multi-finger scaling of the left hand but } \\
\text { bimanual one-finger still used } \\
\text { - Unimanual two finger (index and thumb) rotation } \\
\text { Arm continues resting on side of table unless needed for bimanual } \\
\text { scaling }\end{array}$ \\
\hline 4 from $18-23$ & $\begin{array}{l}\text { - Left arm no longer rest } \\
\text { - Bimanual one-finger scaling } \\
\text { Unimanual two finger (index and thumb) rotation and also } \\
\text { bimanual one-finger rotation }\end{array}$ \\
\hline 5 from $24-29$ & $\begin{array}{l}\text { - } \quad \text { Behavior from } 4 \text { maintained } \\
\text { Elbows resting edge of table for both sides at beginning then } \\
\text { return to left arm resting on side }\end{array}$ \\
\hline
\end{tabular}




\begin{tabular}{|c|c|}
\hline 6 from $30-35$ & $\begin{array}{ll}\text { - } & \text { Right elbow resting } \\
\text { - } & \text { Bimanual one-finger scaling } \\
\text { - } & \text { Unimanual one-finger rotation } \\
\text { - } & \text { Also bimanual one-finger translation } \\
\text { - } & \text { Also unimanual two finger (index and thumb) scaling } \\
\text { - } & \text { Half way through, weight resting on left arm (pic) } \\
\text { - } & \text { Yet, bimanual and unimanual behavior maintained } \\
\end{array}$ \\
\hline 7 from $36-41$ & $\begin{array}{ll}\text { - } & \text { Behavior from } 6 \text { maintained } \\
\text { - } & \text { But moved from resting occasionally }\end{array}$ \\
\hline 8 from $42-47$ & - $\quad$ Behavior from 7 maintained \\
\hline 9 from $48-53$ & - $\quad$ Behavior from 8 maintained \\
\hline 10 from $54-59$ & - $\quad$ Behavior from 9 maintained \\
\hline \multicolumn{2}{|r|}{$\begin{array}{l}\text { Subject } 5 \\
\end{array}$} \\
\hline 1 from $1-5$ & $\begin{array}{l}\text { - Unimanual one-finger rotation by both hands } \\
\text { - } \quad \text { Bimanual one-finger scaling } \\
\text { - } \quad \text { Unimanual one-finger RTS } \\
\text { - Unimanual one-finger translation by both hands }\end{array}$ \\
\hline 2 from $6-11$ & $\begin{array}{ll}\text { - } & \text { Behavior from } 1 \text { maintained } \\
\text { - Occasionally resting arms or palms on table }\end{array}$ \\
\hline 3 from $12-17$ & $\begin{array}{l}\text { - } \quad \text { Behavior from } 2 \text { maintained } \\
\text { In some occasion, piece translated closer to resting on palm left } \\
\text { hand to bimanually one-finger scale }\end{array}$ \\
\hline 4 from $18-23$ & $\begin{array}{l}\text { - } \quad \text { Behavior from } 3 \text { maintained } \\
\text { Sometimes, working bimanually while both elbows are resting on } \\
\text { table }\end{array}$ \\
\hline 5 from $24-29$ & $\begin{array}{ll}- & \text { Behavior from } 4 \text { mostly maintained } \\
\text { - } & \text { Couple of instance of unimanual two finger }(2 \text { and } 3 \text { scaling }) \\
\end{array}$ \\
\hline 6 from $30-35$ & $\begin{array}{ll}- & \text { Behavior from } 5 \text { maintained } \\
\text { - } & \text { Sometimes arm resting on lap when not used } \\
\end{array}$ \\
\hline 7 from $36-41$ & $\begin{array}{ll}\text { - } & \text { Behavior changed from } 6 \\
\text { - } & \text { Unimanual one-finger or two finger }(2-\text { and } 3) \text { rotation } \\
\text { - } & \text { Bimanuanual one-finger translation } \\
\text { - } & \text { Unused hand resting on lap } \\
\end{array}$ \\
\hline 8 from $42-47$ & $\begin{array}{l}\text { - } \begin{array}{l}\text { Behavior from } 7 \text { maintained } \\
\text { Pieces are translated to where the left resting hand is to be } \\
\text { bimanually one-finger scaled }\end{array}\end{array}$ \\
\hline 9 from $48-53$ & $\begin{array}{l}\text { - } \text { Behavior from } 8 \text { maintained } \\
\text { - Larger pieces scaled bimanually one-finger, smaller piece } \\
\text { unimanually two fingers }(2 \text { and } 3) \text { but sometimes even small pieces } \\
\text { bimanually one-finger scaled }\end{array}$ \\
\hline 10 from 54-59 & - $\quad$ Behavior from 9 maintained \\
\hline \multicolumn{2}{|r|}{ Vertical } \\
\hline \multicolumn{2}{|r|}{ Subject 1} \\
\hline 1 from $1-5$ & $\begin{array}{l}\text { - Unimanual one-finger and multi-finger translation from each hand } \\
\text { - } \quad \text { Unimanual multi-finger rotation from each hand } \\
\text { - Unimanual multi-finger RTS and also bimanual multi-finger } \\
\text { - } \quad \text { Bimanual multi-finger scaling } \\
\text { - Arms resting on lap when not in use }\end{array}$ \\
\hline 2 from $6-11$ & $\begin{array}{ll}\text { - } & \text { Behavior from } 1 \text { maintained } \\
\text { Sometimes cross legged with arm resting on one knee when not in } \\
\text { use }\end{array}$ \\
\hline 3 from $12-17$ & - $\quad$ Behavior from 2 maintained \\
\hline 4 from $18-23$ & $\begin{array}{ll} & \text { Behavior from } 3 \text { maintained } \\
\text { - } & \text { RTS mostly carried out bimanually with multi-fingers }\end{array}$ \\
\hline
\end{tabular}




\begin{tabular}{|c|c|}
\hline 5 from $24-29$ & $\begin{array}{l}\text { - Behavior from } 4 \text { maintained } \\
\text { - No cross leg on this one }\end{array}$ \\
\hline 6 from $30-35$ & $\begin{array}{ll}- & \text { Behavior from } 5 \text { maintained } \\
\text { - } & \text { But bimanual one-finger scaling and rotation is noted sometimes }\end{array}$ \\
\hline 7 from $36-41$ & - $\quad$ Behavior from 6 maintained \\
\hline 8 from $42-47$ & - $\quad$ Behavior from 7 maintained \\
\hline 9 from $48-53$ & - $\quad$ Behavior from 8 maintained \\
\hline 10 from $54-59$ & - $\quad$ Behavior from 9 maintained \\
\hline \multicolumn{2}{|r|}{ Subject 2} \\
\hline 1 from $1-5$ & $\begin{array}{ll}\text { - } & \text { Bimanual one-finger scaling } \\
\text { - } & \text { Unimanual one-finger translation from each hand } \\
\text { - } & \text { Bimanual one-finger RTS } \\
\end{array}$ \\
\hline 2 from $6-11$ & - $\quad$ Behavior from 1 maintained \\
\hline 3 from $12-17$ & - $\quad$ Behavior from 2 maintained \\
\hline 4 from $18-23$ & $\begin{array}{ll}\text { - } & \text { Behavior from } 3 \text { maintained } \\
\text { - } & \text { One noted instance of unimanual two finger }\left(2+\text { and } 3^{*}\right) \text { scaling }\end{array}$ \\
\hline 5 from $24-29$ & $\begin{array}{ll}\text { - } & \text { Behavior from } 4 \text { maintained } \\
\text { - } & \text { With also one instance of unimanual two finger scaling } \\
\end{array}$ \\
\hline 6 from $30-35$ & $\begin{array}{ll}- & \text { Behavior from } 5 \text { maintained } \\
\text { - } & \text { No unimanual scaling } \\
\end{array}$ \\
\hline 7 from $36-41$ & - $\quad$ Behavior from 6 maintained \\
\hline 8 from $42-47$ & - $\quad$ Behavior from 7 maintained \\
\hline 9 from $48-53$ & - $\quad$ Behavior from 8 maintained \\
\hline 10 from $54-59$ & - $\quad$ Behavior from 9 maintained \\
\hline \multicolumn{2}{|r|}{ Subject 3} \\
\hline 1 from $1-5$ & $\begin{array}{l}\text { - Unimanual two finger (index and thumb) scaling from each hand \& } \\
\text { bimanual one-finger scaling, but mostly unimanual } \\
\text { - Unimanual one-finger translation from each hand } \\
\text { - } \text { Bimanual one-finger RTS } \\
\text { - Unimanual two finger (index and thumb) also }(2 \text { and } 3 \text { finger) } \\
\text { - } \text { Alternating between hands } \\
\text { When not in use arm resting on lap }\end{array}$ \\
\hline 2 from $6-11$ & $\begin{array}{ll}\text { - } & \text { Unimanual one-finger translation from each hand } \\
\text { - } & \text { Bimanual one-finger scaling } \\
\text { - } & \text { Bimanual one-finger RTS } \\
\text { - } & \text { Unimanual two finger }\left(2 \text { and } 3{ }^{*}\right) \text { rotation } \\
\text { - } & \text { Alternating between hands } \\
\text { - } & \text { When not in use arm resting on lap } \\
\end{array}$ \\
\hline 3 from $12-17$ & 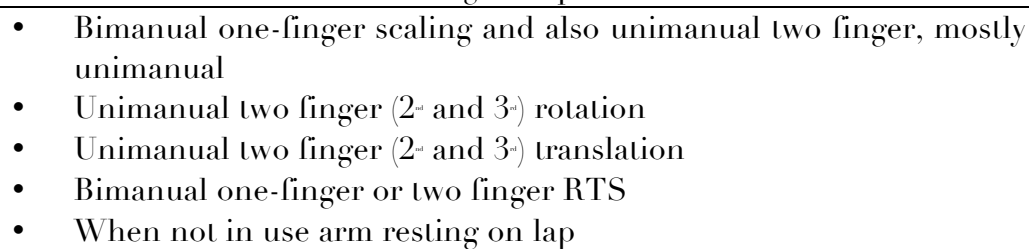 \\
\hline 4 from $18-23$ & - $\quad$ Behavior from 3 maintained \\
\hline 5 from $24-29$ & $\begin{array}{ll}- & \text { Behavior from } 4 \text { maintained } \\
\text { - } & \text { One instance of bimanual two finger }(2 \text { and } 3-) \text { rotation }\end{array}$ \\
\hline 6 from $30-35$ & - $\quad$ Behavior from 5 maintained \\
\hline 7 from $36-41$ & - $\quad$ Behavior from 6 maintained \\
\hline 8 from $42-47$ & - $\quad$ Behavior from 7 maintained \\
\hline 9 from $48-53$ & - $\quad$ Behavior from 8 maintained \\
\hline 10 from $54-59$ & $\begin{array}{l}\text { - } \quad \text { Behavior from } 9 \text { maintained } \\
\text { Rarely any bimanual in the beginning, but increased slightly at the } \\
\text { end }\end{array}$ \\
\hline
\end{tabular}




\begin{tabular}{|c|c|}
\hline \multicolumn{2}{|r|}{ Subject 4} \\
\hline 1 from $1-5$ & $\begin{array}{l}\text { - Unimanual one-finger translation for each hand, mostly right } \\
\text { - Unimanual two finger (index and thumb) scaling for each hand, } \\
\text { mostly right } \\
\text { - Unimanual two finger (index and thumb) rotation, for each hand } \\
\text { mostly right } \\
\text { - Unimanual two finger RTS etc. } \\
\text { Unused arm resting on lap }\end{array}$ \\
\hline 2 from $6-11$ & $\begin{array}{ll}\text { - } & \text { Behavior from } 1 \text { maintained } \\
\text { - } & \text { But bimanual one-finger RTS used occasionally }\end{array}$ \\
\hline 3 from $12-17$ & $\begin{array}{ll}- & \text { Bimanual one-finger scaling } \\
\text { - } & \text { Other than that behavior from } 2 \text { maintained }\end{array}$ \\
\hline 4 from $18-23$ & - $\quad$ Behavior from 3 maintained \\
\hline 5 from $24-29$ & $\begin{array}{ll}- & \text { Behavior from } 4 \text { maintained } \\
\text { - } & \text { Cross legged with unused arm resting on it }\end{array}$ \\
\hline 6 from $30-35$ & $\begin{array}{ll} & \text { Behavior from } 5 \text { maintained } \\
\text { - } & \text { Unimanual two finger (index and thumb) noted for RTS }\end{array}$ \\
\hline 7 from $36-41$ & - $\quad$ Behavior from 6 maintained \\
\hline 8 from $42-47$ & - $\quad$ Behavior from 7 maintained \\
\hline 9 from $48-53$ & $\begin{array}{ll}\text { - } & \text { Behavior from } 8 \text { maintained } \\
\text { - } & \text { Unused arm has weight resting on it on the chair }\end{array}$ \\
\hline 10 from $54-59$ & - $\quad$ Behavior from 9 maintained \\
\hline \multicolumn{2}{|r|}{$\begin{array}{c}\text { Subject } 5 \\
\end{array}$} \\
\hline 1 from $1-5$ & $\begin{array}{ll}\text { - } & \text { Unimanual one-finger translation from each hand } \\
\text { - } & \text { Bimanual one-finger scaling } \\
\text { - } & \text { Bimanual one-finger RTS } \\
\text { - } & \text { When not in use arm resting on lap } \\
\end{array}$ \\
\hline 2 from $6-11$ & $\begin{array}{l}\text { - } \quad \text { Behavior similar to } 1 \\
\text { - } \quad \text { But unimanual RTS is noted with two fingers }(2 \text { and } 3) \\
\text { - Also occasionally unimanual two fingers }(2 \text { and } 3 * \text { rotation and } \\
\text { scaling }\end{array}$ \\
\hline 3 from $12-17$ & • $\quad$ Behavior from 2 maintained \\
\hline 4 from $18-23$ & $\begin{array}{ll}\text { - } & \text { Behavior from } 3 \text { maintained } \\
\text { - } & \text { Two fingers used also are index and thumb }\end{array}$ \\
\hline 5 from $24-29$ & - $\quad$ Behavior from 4 maintained \\
\hline 6 from $30-35$ & - $\quad$ Behavior from 5 maintained \\
\hline 7 from $36-41$ & - $\quad$ Behavior from 6 maintained \\
\hline 8 from $42-47$ & - $\quad$ Behavior from 7 maintained \\
\hline 9 from $48-53$ & - $\quad$ Behavior from 8 maintained \\
\hline 10 from $54-59$ & - $\quad$ Behavior from 9 maintained \\
\hline \multicolumn{2}{|r|}{ Subject 6} \\
\hline 1 from $1-5$ & $\begin{array}{l}\text { - } \quad \text { Bimanual multi-finger and one-finger scaling, mostly one-finger } \\
\text { - } \quad \text { Unimanual one-finger translation from each hand } \\
\text { - } \quad \text { Bimanual one-finger RTS } \\
\text { - } \quad \text { When hand one-finger rotation } \\
\end{array}$ \\
\hline 2 from $6-11$ & - $\quad$ Behavior from 1 maintained \\
\hline 3 from $12-17$ & $\begin{array}{ll}- & \text { Behavior from } 2 \text { maintained } \\
\text { - } & \text { Cross legged behavior for arm to rest when not in used is noted }\end{array}$ \\
\hline 4 from $18-23$ & - $\quad$ Behavior from 3 maintained \\
\hline 5 from $24-29$ & - $\quad$ Behavior from 4 maintained \\
\hline 6 from $30-35$ & - $\quad$ Behavior from 5 maintained \\
\hline 7 from $36-41$ & - $\quad$ Behavior from 6 maintained \\
\hline 8 from $42-47$ & - $\quad$ Behavior from 7 maintained \\
\hline 9 from $48-53$ & - $\quad$ Behavior from 8 maintained \\
\hline 10 from $54-59$ & - $\quad$ Behavior from 9 maintained \\
\hline
\end{tabular}

